\title{
Electrical Determination of Vortex State in Submicron Magnetic Elements
}

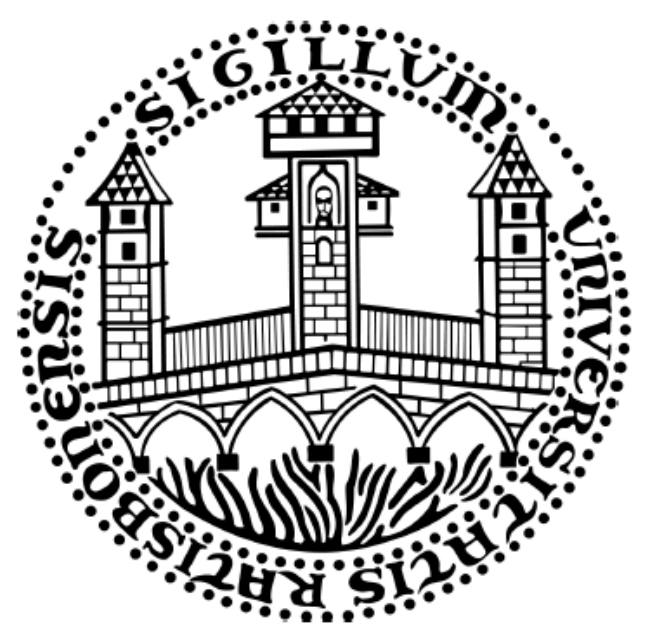

Dissertation

zur Erlangung des Doktorgrades der Naturwissenschaften

(Dr. rer. nat.)

der Fakultät für Physik

der Universität Regensburg

vorgelegt von

\author{
Ajay Gangwar \\ M.Sc. (IIT Kanpur) \\ geboren am 05.04.1986 \\ aus Pilibhit, India
}

März 2015 
Promotionsgesuch eingereicht am: 12.03.2015

Die Arbeit wurde angeleitet von: Prof. Dr. Christian H. Back

$\begin{array}{lll}\text { Prüfungsausschuss: } & \text { Vorsitzender: } & \text { Prof. Dr. T. Wettig } \\ & \text { 1. Gutachter: } & \text { Prof. Dr. C. H. Back } \\ \text { 2. Gutachter: } & \text { Prof. Dr. G. Schütz } \\ & \text { weiterer Prüfer: } & \text { Prof. Dr. D. Weiss }\end{array}$




\section{Abstract}

Vortex structures in confined geometries are currently under close scrutiny due to their unique properties associated with their spatial confinement and the nonuniform distribution of the magnetization. The magnetic vortex is characterized by two boolean topological quantities: circulation (clockwise or counterclockwise $c= \pm 1$ ) of the in-plane magnetization and polarity (up or down, $p= \pm 1$ ) of the vortex core. These four degenerate states are quite stable and can accelerate the development of more compact and high performance magnetic memory devices. Thus an understanding of their dynamical behavior and a way to electrically detect these states is a major requirement for their development. Progress can be achieved by combining theoretical calculations, micromagnetic simulations and experimental approaches. The phenomenon of spin transfer torque is exploited to excite the lowest frequency (gyro) mode of the vortex core confined in a submicron magnetic (Permalloy - Ni $i_{81} F e_{19}$ ) element. The gyrotropic motion of the vortex core leads to a periodic change in the magnetization and hence its resistance: due to the anisotropic magnetoresistance (AMR) effect. This periodic change in resistance combines with the excitation current and generates a periodic homodyne voltage signal. An external static magnetic field is applied to break the symmetry and to rectify the homodyne voltage signal which we measure in a nanovoltmeter. It is found that the sign of the rectified AMR signal depends upon the handedness $(c p)$ of the vortex structure. Micromagnetic simulations provide better understanding and are in good agreement with our experimental results. Additionally, vortex dynamics in these samples is investigated in a Scanning Transmission X-ray Microscope (STXM) with a temporal $(<100 \mathrm{ps})$ and spatial $(\leqslant 30 \mathrm{~nm})$ resolution which allows us to verify the resonance frequency of the magnetic element as well as the power range to excite the vortex core. The AMR based technique thus can be used to detect the circulation and the polarity of the vortex state electrically and could open a route to implement magnetic vortex elements in memory and storage hierarchies.

The phenomenon of Spin Motive Force (SMF) has also been studied by micromagnetic simulations. It is found that, in a particular configuration, the SMF signal shows a phase difference of 180 degrees for two polarities of the vortex core, when the voltage probe contacts are located parallel to the excitation rf field direction. No phase shift is observed in the perpendicular case. In addition, a 180 degree phase difference is observed for different circulations of the vortex structure. 
Therefore, this could also be a possible way to determine polarity and circulation of the magnetic vortex by carefully examining the phase relation of the SMF generated voltage signals. An attempt has also been made to measure the SMF experimentally. However, due to the small expected signal unambiguous detection of SMF was not successful so far. 
Dedicated to my parents 


\section{Acknowledgment}

I am privileged to complete my PhD thesis here at University of Regensburg. The sustained success of my thesis was possible only through the dedication of a large team of people who have generously supported me throughout the process of my thesis. Therefore I want to use this opportunity to extend my profound appreciation for a number of people, some for directly contributing to this work, others simply for creating a pleasant environment to live and work.

First and foremost, I would like to express my sincere gratitude to my supervisor Prof. Dr. Christian H. Back for giving me an opportunity to work in his supervision. His unending patience and enthusiasm, even in the face of delays and dead ends, have helped me to make these years a true learning experience. He has always made my life easier e.g. the seemingly infinite loops of making changes to conference abstracts, paper and (of course) this thesis. His invaluable help and support made me to learn lot of things and I will profit from that over my entire career.

I would like to gratefully acknowledge Dr. Hermann Stoll for his enthusiastic discussions to clear my doubts specially about high frequency circuits. Eventhough, I didn't got much opportunities to spend time with him but I always learned something whenever we met and enjoyed discussions over phone and also via long emails. I owe him lot of thanks for his support and hard work. I would also like to thank Prof. Dr. Georg Woltersdorf, who has chosen me to start my PhD here in Regensburg. I have learned many things from him starting from device fabrication to building up the experimental setups and performing experiments independently. I want to thank Prof. Dr. Gisela Schütz for considering me as a part of her group and for involving me in some of her research projects. I would like to thank her for her direct and indirect support whenever I need it.

Let me thank to Dr. Hans G. Bauer, without whom getting my PhD would have been impossible, or at least a lot less fun. I really owe him lot for making me to understand many things in physics and helping me with micromagnetic simulations. I am (and probably many others are also) impressed by his insight and skills of micromagnetic simulations. I would also like to thank Dr. Jean-Yves Chauleau for starting the project related to AMR measurements and his valuable suggestions and comments during this project. I have had several office mates - Hans, Helmut, Dahai and more. I have enjoyed their companionship and also had a memorable two week summer school in Nijmegen with Helmut and Robert. Not only the part of the office, but also the part of the group with whom I talked about both physics and 
non-physics made my days memorable. You guys have been my family for these years, and will always be my friends. My acknowledgement to all my colleagues who helped me and all who have contributed to a nice atmosphere of our group. Although, it's time to move forward in our life, I will remember these precious moments spent with you all and will miss you all and your friendship.

I would like to thank Dr. Markus Weigand and Michael Bechtel for their support with STXM setup and measurements at BESSY. I would like to thank Matthias Noske for being there whenever I need him to help me with the STXM measurements. He was really great during my beamtimes and cleared many doubts even when he was not present there personally. Here, I should also mention Dr. Markus Sproll, Georg Dieterle, Michael Vogel, Susanne Brunner and Iulia with whom I enjoyed some beamtimes. I would also like to thank the BESSY staff for their continuous support and the HZB for the allocation of beamtimes.

Further, I want to thank Markus Hollnberger and Dieter Schierl for their assistance with all types of technical problems. Besides your technical knowledge, you guys bring lots of life to the group and you are of great importance for the good atmosphere. I would like to thank our secretaries Sylvia Hrdina, Claudia Zange, Monika Kotz, Claudia Rahm and Edeltraud Schlagbauer for taking care of administrative work and many other things. I would like to extend my acknowledgement to the people from mechanical and electronic workshops. I want to mention here Dieter Riedl and his team for their support on the home-built electronics and to solve many other electronics problems.

I should acknowledge the great effort of our collaborators and research fellows who also made contributions to complete my PhD work. I would like to thank Prof. Dr. Dieter Weiss for allowing me to access the clean room facilities. Research Group of Prof. Weiss is thanked for the technical support during sample preparation in the clean room and general advice; in particular I would like to acknowledge the help of Cornelia Linz.

As is custom in physics literature, I would now like to acknowledge my sources of funding. Financial support for this work was derived in part from SFB-689 and from the research budget of DFG.

Finally, I am forever indebted to my parents for their understanding, endless patience and encouragement whenever it was required. You have stood by me throughout and getting where I am today would have been impossible without the full support of my parents. 


\section{Contents}

$\begin{array}{ll}\text { Abstract } & 3\end{array}$

$\begin{array}{lr}\text { Acknowledgment } & 6\end{array}$

$\begin{array}{lll}1 & \text { Introduction } & 10\end{array}$

2 Theoretical Background $\quad 14$

2.1 Magnetization Dynamics . . . . . . . . . . . . . . . . . 14

2.2 Vortices in Magnetic Microstructures and their Dynamics . . . . . . 19

2.2.1 Vortices in an External Static Field . . . . . . . . . . . . 20

2.2.2 Field Induced Vortex Dynamics . . . . . . . . . . . . . . 21

2.2 .3 Current Induced Vortex Dynamics . . . . . . . . . . . . . 22

2.2.4 Vortex Core Switching . . . . . . . . . . . . . . 23

2.2.5 Polarization Selective Switching . . . . . . . . . . . . 24

2.3 Spin Waves . . . . . . . . . . . . . . . . . 26

2.3.1 Spin Waves in Magnetic Vortex Structures . . . . . . . . 28

3 Detection Methods and Micromagnetic Simulations 31

3.1 Imaging Methods . . . . . . . . . . . . . . . . . . 31

3.2 Electrical Detection . . . . . . . . . . . . . . . . . 33

3.2.1 AMR in Vortex Structures . . . . . . . . . . . . . . . . 33

3.2.2 Micromagnetic Simulations (AMR) . . . . . . . . . 36

3.2 .3 Spin Motive Force . . . . . . . . . . . . . . . . . . . . 40

3.2.4 Micromagnetic Simulations (SMF) . . . . . . . . . . . 41

3.3 Exchange Bias . . . . . . . . . . . . . . . . . . . . . 44

4 Sample Preparation $\quad 45$

4.1 Methods and Techniques . . . . . . . . . . . . 46

4.1 .1 Optical Lithography . . . . . . . . . . . . . . . . 46

4.1.2 Electron Beam Lithography . . . . . . . . . . . . . . . 49

4.1.3 Metal Deposition Techniques. . . . . . . . . . . . 53

4.1.4 Atomic Layer Deposition . . . . . . . . . . . . . . . . . . . . 55

4.2 Fabrication Steps and Samples for Different Experiments . . . . . . 56 
5 Instrumentation and Measurement Techniques $\quad 63$

5.1 Scanning Transmission X-ray Microscopy . . . . . . . . . . . . 63

5.1.1 Synchrotron and Generation of X-rays . . . . . . . . . 63

5.1 .2 Contrast Mechanism: The XMCD Effect . . . . . . . . . . 64

5.1 .3 STXM Setup . . . . . . . . . . . . 66

5.2 Electrical Measurement Setup . . . . . . . . . . . . . 68

5.2 .1 AMR Setup . . . . . . . . . . . . . . . 68

$5.2 .2 \quad$ SMF Setup . . . . . . . . . . . . . . . 71

6 Results $\quad 73$

6.1 STXM Measurements . . . . . . . . . . . . . . . . . 73

6.1 .1 Static Imaging . . . . . . . . . . . . . . 73

6.1 .2 Dynamic Imaging . . . . . . . . . . . . . . . . . 76

6.2 AMR Measurements . . . . . . . . . . . . . . . . . . . 79

6.2 .1 DC AMR . . . . . . . . . . . . . . . . . 79

6.2.2 Homodyne Detection . . . . . . . . . . . . . . . . 81

6.3 Other Experiments . . . . . . . . . . . . . . . 86

$\begin{array}{lll}7 & \text { Summary } & 89\end{array}$

$\begin{array}{ll}\text { Publications } & 91\end{array}$

A Thiele Equation $\quad 92$

$\begin{array}{ll}\text { B SMF Equation } & 95\end{array}$

$\begin{array}{ll}\text { Bibliography } & 98\end{array}$ 


\section{Introduction}

An electron, along with the charge, is a carrier of the spin degree of freedom which has been studied intensely in the last century. Spins are the fundamental magnetic entities in solids, and what determines a material's magnetic state is how electron spins are arranged. If most of the spins point in the same direction, the material is ferromagnetic, like a refrigerator magnet. If half the spins point one way and half the opposite, the material is antiferromagnetic, with no effective magnetization. For many decades, studies of different magnetic systems have become of large interest, in particular with respect to industrial applications [1, 2]. Metallic magnetism has a long history because there have been continuous discoveries of many intriguing phenomena and difficulties in their theoretical description. In the field of magnetic materials, a rapidly expanding area of study concerns stable nanometer scale spin arrangements in metals [3]. In recent decades there is a considerable interest in research and development in the field of nanoscale magnetism, which is one of the most prospective fields in today's science and ground for new branches of high-tech industry $[4,5,6,7]$. The intensive research based on spin-polarized and spin dependent transport processes in low dimensional nanoscale systems have promoted considerable progress in technological applications in new generation microelectronics devices with powerful functionality, and fast processing speed. Nanoscale magnetic materials have already found applications in various areas such as information technology, magnetosensors, electronics, data storage, magnetic read heads of computer hard disks, single electron devices, microwave electronic devices, advanced filters for telecommunications etc. [1, 2, 4, 6, 8].

For about ten years now, tiny magnetic structures measuring a few millionths of a millimeter have met with growing interest from the worlds of science and technology, particularly on account of their potential applications in magnetic data storage [1, $2,7]$. The evolution of digital electronics is a story of miniaturization and each generation of circuitry requiring less space and energy to perform the same tasks. Even high speed processors move into hand-held smart phones, though current data storage technology has a functional limit. With the continuing miniaturization, the interaction between neighbouring bits increases due to magnetic stray fields 
which can lead to loss of data. In addition, small magnetic bits are less stable against thermal fluctuations which is also called the super-paramagnetic limit [9]. Therefore, the increase of the data storage capacity (decreasing the magnetic unit dimensions down to micro- or nano-meters) and the decrease of the access times (of nanoseconds and sub nanoseconds for MRAM devices), are the major challenges of current research work. To mobilize this emerging technology, we must understand the fundamentals of magnetization dynamics in micro and nano scale structures and learn how to manipulate bits of magnetic information as a reliable carrier of computer code.

In recent years, artificial mesoscopic magnetic systems with a confined structure have had a great impact in research area because of their possible applications in magnetic data storage and information processing $[5,7,10]$, single magnetic nanoparticle sensing and trapping [8, 11], microwave-frequency oscillators [12, 13], or frequency multiplication [14]. Another reason is the very rich physics of these systems, originating from the competition between the exchange and dipolar interactions, the effect of which is increased by the minute size of the systems. A fascinating phenomenon that occurs in these confined magnetic structures is the so called vortex state, which has been predicted in theory long ago, but was experimentally observed in the last decade only $[15,16]$. While considerable attention has been devoted to their transport properties, the radio frequency $(\mathrm{RF})$ response of these systems has garnered interest as well. This research contribution has concentrated on the study of confined magnetic structures in the vortex state, especially, on fabrication, characterization, physics behind the unique properties of these structures and device design. As a result of this research, it is possible to obtain guidelines for the realization of a technology for manipulating the states of magnetic information media with extremely low power consumption. We have studied vortex dynamics excited by rf magnetic fields and currents in great detail with special emphasis on the detection of the vortex state electrically exploiting a well known effect, the so called Anisotropic Magnetoresistance (AMR) effect and the newly discovered Spin Motive Force (SMF) phenomenon.

This thesis is organised in the following way:

Chapter-2 describes theoretical background of magnetization dynamics excited with magnetic fields and with electrical currents as well. Theory will be extended to describe the dynamics in confined magnetic structures in the vortex state. Controlling the vortex states and effective switching of the vortex core polarity will be explained. Some of the pioneering research work in this field performed earlier 
in our research group will also be included in this chapter. Addionally for completeness, it is intended to address spin waves in magnetic systems and spin wave assisted switching in the vortex structures.

Chapter-3 presents different methods to investigate the magnetization state and its dynamics in nanoscale magnetic systems. A short summary of some of the time - and space - resolved microscopic imaging techniques used to visualize and characterize the magnetization and its dynamics in nanostructures will be provided along with their capability and limitations. Special emphasis will be given to the STXM technique which is more relevant to this work and used to investigate vortex dynamics. Apart from the imaging techniques, we will focus on electrical determination of the vortex state by Anisotropic Magnetoresistance (AMR) and Spin Motive Force (SMF) which have been studied during the course of this research work. A theoretical background of these two effects will be presented to understand the origin and importance of these effects. It explains basic concepts needed to understand the results presented in this thesis. Micromagnetic modelling and simulations based on the Landau-Lifshitz-Gilbert equation of motion of magnetization are also discussed, highlighting how the micromagnetic computer simulation approach contributes to an understanding of spin dynamics of nanomagnetism and considering some of the merits of numerical simulation studies. Some results of the micromagnetic simulations performed on magnetic vortex structures will also be presented to get better understanding of the AMR and SMF effects and to support and validate our experimental results.

Chapter-4 gives a detailed description of different techniques and processes involved to prepare samples during my research work. Optical and elecron-beamlithography techniques used to pattern structures on the micro - and nano - scale will be described in detail. Methods of thin film preparation using different Physical Vapour Deposition (PVD) techniques will be described along with its benefit and precautions in choosing a suitable method. Afterwards, the device fabrication will be described step by step. The chapter will end up with a description of different types of samples prepared for several other projects in collaboration with other groups.

Chapter-5 describes different measurement techniques used to perform experiments during the experimental work presented in this thesis. A summary of Scanning Transmission X-ray Microscopy, along with the instrumentation and measurement setup at MAXYMUS endstation of BESSY II will be presented. The contrast mechanism XMCD used to image our magnetic structures will be described and the 
different configurations used to measure in-plane and out-of-plane magnetization in the sample will also be explained. The measurement setup, at the University Regensburg, used to investigate magnetic vortices electrically utilizing AMR and SMF phenomenon will be described in detail. The functioning of different rf-components will also be mentioned whenever it is required.

Chapter-6 reports on the results obtained from the experiments performed during this research work and the comparison with our micromagnetic simulations. The vortex structures are first characterised, by directly looking at their magnetization and dynamics in STXM. The response of the vortex structure and its core to external magnetic fields and rf currents is measured which is used later on for electrical measurements. The results obtained from AMR measurements on vortex structures are presented and prove that the vortex state can be determined by these measurements. Here we also took advantage of micromagnetic simulations to explain our experimental results and many features e.g. the shift in resonance frequency of the vortex core with increasing external magnetic fields. In the last section of this chapter, a summary of the experiments and obtained results for different samples will be presented.

Chapter-7 concludes this thesis with a short summary of this work. 


\section{Theoretical Background}

\subsection{Magnetization Dynamics}

Each magnetic system is characterized by its equilibrium and metastable magnetic states, corresponding to respective local minima in the total energy of the magnetic system. The transition of the magnetization from one state to another, under the influence of an external magnetic field, temperature or a spin polarized current is a dynamic process, called magnetization dynamics, whose characteristics depends upon the type of excitation, the material parameters as well as the spatial dimensions of the magnetic system [3]. In order to describe the dynamics of the magnetization processes Landau and Lifshitz were the first to derive a mathematical expression in the form of a differential equation [17]. It has later been extended by Gilbert and the corresponding equation of motion for the magnetization $(\vec{M})$ dynamics induced by an effective magnetic field $\left(\vec{H}_{e f f}\right)$ is nowadays known as the Landau-Lifshitz-Gilbert equation [18]:

$$
\frac{d \vec{M}}{d t}=-|\gamma| \vec{M} \times \vec{H}_{e f f}+\frac{\alpha}{M_{s}} \vec{M} \times \frac{d \vec{M}}{d t}
$$

The so-called LLG equation describes the dynamics of individual magnetic moments interacting with each other through exchange interaction, dipolar coupling, magnetocrystalline anisotropy and Zeeman field. The first term on the right hand side corresponds to the precession of the magnetization around the effective magnetic field (see Fig. 2.1). This term conserves the energy of the system. The precession rate is proportional to the field $\vec{H}_{e f f}$, with a proportionality factor $\gamma-$ the gyromagnetic ratio which is given by:

$$
\gamma=\frac{\mu_{0} g e}{2 m_{e}}
$$

where $\mathrm{g} \approx 2$, is the Landé $\mathrm{g}$ - factor, $\mu_{0}$ is the permeability of free space, e and $m_{e}$ are the charge and mass of the electron, respectively. The second term in eq - [2.1] is the damping term which is of a purely phenomenological origin and 


\subsection{Magnetization Dynamics}

describes the energy loss during magnetization precession, attributed to the magnon phonon coupling and the generation of eddy currents. These are simply modelled as a dissipation term proportional to the precession rate, with an empirical dimensionless proportionality factor " $\alpha$ " called damping parameter which determines how fast the magnetization relaxes towards the direction of $\vec{H}_{e f f}$. The damping parameter can be determined experimentally e.g. from the ferromagnetic resonance (FMR) linewidth. We note that the LLG equation is a non-linear equation and thus one may expect complicated dynamics in some range of dynamic parameters.

We can transform the LLG equation in

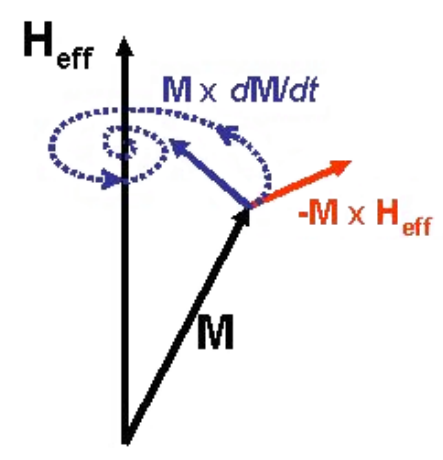

Figure 2.1: The terms of the Landau Lifshitz Gilbert equation: precession (red) and damping (blue). The trajectory of the magnetization (dotted spiral) is drawn under the simplifying assumption that the effective field $\left(\vec{H}_{\text {eff }}\right)$ is constant.

an alternative form in terms of different types of fields governing the magnetization dynamics as following [19]:

Assuming the length of the magnetization vector is not changing during the precessional motion i.e. $|\vec{M}|=M_{s}=$ constant or $\vec{M} \cdot \vec{M}=M_{s}^{2}=$ constant,

$$
\Rightarrow \frac{d M_{s}^{2}}{d t}=0
$$

Then, we can write

$$
\begin{gathered}
\vec{M} \times\left(\vec{M} \times \frac{d \vec{M}}{d t}\right)=\vec{M}\left(\vec{M} \cdot \frac{d \vec{M}}{d t}\right)-M_{s}^{2} \frac{d \vec{M}}{d t}=\vec{M}\left(\frac{1}{2} \frac{d M_{s}^{2}}{d t}\right)-M_{s}^{2} \frac{d \vec{M}}{d t}=-M_{s}^{2} \frac{d \vec{M}}{d t} \\
\Rightarrow \frac{d \vec{M}}{d t}=-\frac{1}{M_{s}^{2}} \vec{M} \times\left(\vec{M} \times \frac{d \vec{M}}{d t}\right)
\end{gathered}
$$

substituting the left hand side of equation - [2.1] by the above expression, we get

$$
\begin{aligned}
& -\frac{1}{M_{s}^{2}} \vec{M} \times\left(\vec{M} \times \frac{d \vec{M}}{d t}\right)=-|\gamma| \vec{M} \times \vec{H}_{e f f}+\frac{\alpha}{M_{s}} \vec{M} \times \frac{d \vec{M}}{d t} \\
& \Rightarrow|\gamma| \vec{M} \times\left[\vec{H}_{e f f}-\frac{1}{|\gamma| M_{s}^{2}}\left(\vec{M} \times \frac{d \vec{M}}{d t}\right)-\frac{\alpha}{|\gamma| M_{s}} \frac{d \vec{M}}{d t}\right]=0
\end{aligned}
$$


Thus we can write the LLG equation as

$$
|\gamma| \vec{M} \times\left(\vec{H}_{e f f}+\vec{H}^{g}+\vec{H}^{d}\right)=0
$$

where $\vec{H}^{g}$ and $\vec{H}^{d}$ are named gyrotropic field and damping field, respectively, defined as

$$
\left\{\begin{array}{l}
\vec{H}^{g}=-\frac{1}{|\gamma| M_{s}^{2}}\left(\vec{M} \times \frac{d \vec{M}}{d t}\right), \\
\vec{H}^{d}=-\frac{\alpha}{|\gamma| M_{s}} \frac{d \vec{M}}{d t}
\end{array}\right.
$$

equation - [2.3] holds if

$$
\vec{H}_{e f f}+\vec{H}^{g}+\vec{H}^{d}=0
$$

Note that the effective field has contributions from both the external and internal fields, such as the demagnetizing field, the effective exchange field and the effective magnetocrystalline anisotropy field.

Recent research on magnetic nanostructures has shown that effects caused by an electric current flowing across a nanostructure may dominate over the effects due to the field generated by the same current. The spin polarized current, a flow of electron spin angular momentum that arises when there is an imbalance between a flow of up and down oriented electron spins, carries a net spin angular momentum which can be transferred to the local magnetization if the polarization direction differs from the spin quantization (magnetization) direction in the ferromagnet. This phenomenon is known as the spin-transfer-torque (STT) effect [20] predicted by Sloncwezski and Berger [21, 22]. This torque is a result of an interaction between itinerant electrons that are spin polarized (spin polarized current) and the local magnetization. The spin-transfer-torque effect can be understood as follows, see Fig. 2.2. The electron spins ( $\hat{\mathbf{s}})$ flowing through a ferromagnet and non-collinear with respect to the magnetization direction, are not eigenstates of the ferromagnet, but can be described as a coherent linear combination of majority $(\uparrow)$ and minority $(\downarrow)$ spins as following:

$$
|\hat{\mathbf{s}}\rangle=\frac{1}{\sqrt{2}}(|\uparrow\rangle \pm|\downarrow\rangle)
$$

An electron flowing through the ferromagnet interacts with the local magnetic moment and this interaction results in a reorientation of the electron spin on transmission through (or reflection from) the ferromagnetic layer, Fig. 2.2 (a). By conservation of angular momentum, a change in the direction of an electron's spin angular 


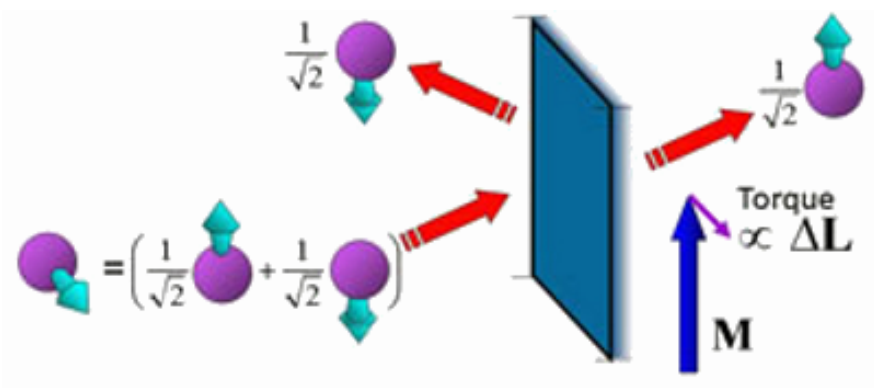

(a)

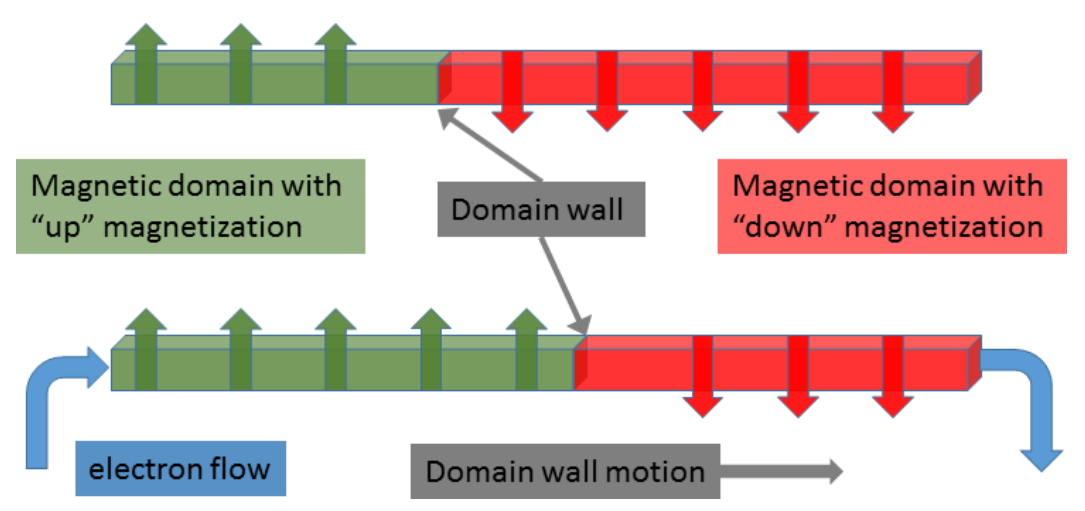

(b)

Figure 2.2: Sketch to understand spin-transfer-torque effect (a) and the magnetization dynamics (domain wall motion) due to this effect.

momentum leads to a torque on the magnetization of the ferromagnet which can either be in the plane of the incoming and outgoing electron spin direction or perpendicular to that plane. The former is often called the in-plane (or adiabatic) spin transfer torque and the latter is known as the perpendicular torque (or nonadiabatic or field like torque). The relative importance and magnitude of the two spin transfer torques depends on the material and the device structure and is critical to applications in that they determine the threshold currents for magnetization switching and magnetization precession frequencies.

Spin transfer torques are not only important in magnetic multilayer systems where the magnetization is uniform within each magnet, but also in systems where the magnetization gradually changes its direction e.g. in domain walls (DW), a boundary between two domains having different magnetization directions. The width of a DW is in the range of 10 to $100 \mathrm{~nm}$ and is determined by the competition between the exchange stiffness that aligns spins and the anisotropy energy that orients spins in energetically favoured directions. Consider spin-polarized itinerant electrons passing through a DW, Fig. 2.2 (b). As the electrons traverse the DW, as 
a result of the exchange interaction with the localized moments, the spin of itinerant electrons rotate and change direction following the direction of the local magnetic moments. The conservation of angular momentum dictates that the change of angular momentum of the traversing electrons is transferred to the local moments and thus exerts a torque on it. This torque changes the direction of local moments which results in a displacement of the DW and this phenomena is called current induced domain wall motion [23, 24, 25]. Thus the magnetization dynamics can be excited through the spin-transfer-torque effect by flowing current through a magnetic stack or magnetic texture [26, 27]. It provides a smart alternative for exciting and switching the magnetization without applying an external magnetic field. This effect has already been applied in magnetic random access memories (MRAM) and is the basis for the spin-transfer-torque oscillators that are envisaged for applications in communication technology.

Some additional terms are required in the LLG equation in order to include the action of a spin polarized current on the magnetization dynamics. The time evolution of the magnetization ( $\vec{M}$ ), excited by the spin-transfer-torque effect, can thus be described by the extended Landau - Lifshitz - Gilbert (LLG) equation including STT terms [28, 25].

$$
\begin{aligned}
\dot{\vec{m}}= & -|\gamma| \vec{m} \times \vec{H}_{e f f}+\alpha \vec{m} \times \dot{\vec{m}} \\
& -(\vec{u} \cdot \vec{\nabla}) \vec{m}+\beta \vec{m} \times[(\vec{u} \cdot \vec{\nabla}) \vec{m}]
\end{aligned}
$$

Here $\vec{m}$ is a unit vector in the direction of $\vec{M}, \vec{u}$ is a vector having units of velocity and directed along the direction of electron motion having magnitude $u=j P g \mu_{B} / 2 e M_{s}$ with current density $\mathrm{j}$, polarization $P$ of the conduction electrons, $\mu_{B}$ the Bohr magneton, $e$ the electron charge and $M_{s}$ the saturation magnetization. The first two terms of the above equation have already been described in equation 2.1. The last two terms describe the magnetization dynamics due to STT effect induced by a current density $\vec{j}[29]$. Here $\beta$ is the non-adiabatic parameter the published values of which vary over a wide range [30, 31, 32, 33] and its exact value is still under debate in the magnetism research community. The results appear to depend strongly on the geometry, the microstructure and the pinning potential due to imperfections in the structures which strongly contribute to the large span of reported values of $\beta$. Theory predicts that both the velocity of a domain wall and the critical current for domain wall movement depend on $\beta$ [25]. Thus, the exact determination of this parameter is crucial for the optimization of material systems for technological purposes. 
(a)

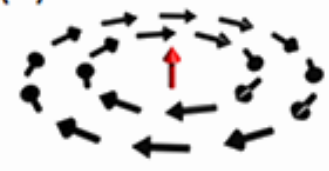

(b)

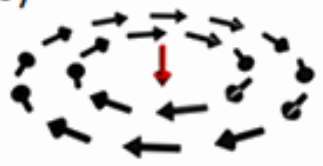

(c)

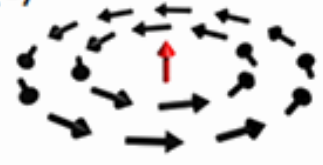

(d)

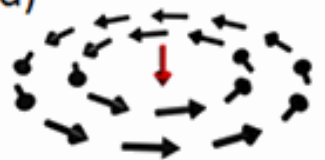

Figure 2.3: Nomenclature of magnetic vortices in micron and submicron magnetic platelets. The magnetic moments away from the center of the structure are aligned parallel to the surface, either clockwise or counterclockwise. The magnetic moments in the core of the vortex point out of the plane, either up or down. The four degenerate magnetic ground states exist in such micromagnets.

\subsection{Vortices in Magnetic Microstructures and their Dynamics}

The magnetic vortex $[34,15,16,35]$ is a typical ground state configuration of micron and sub-micron sized soft ferromagnetic thin film structures. Such elements are magnetized in-plane because of the predominant influence of the stray field energy (shape anisotropy). Surface charges at the boundary of the sample are avoided by aligning the magnetic moments parallel to the edges and due to exchange interactions neighboring spins in the inner region are aligned parallel to those in the outer region resulting in a flux-closure pattern. The sense of the in-plane flux closure is called the circulation $c$ and could take two possible values: $c=+1$ (counterclockwise orientation) and $c=-1$ (clockwise orientation). As we approach the center of the magnetic element neighboring spins would inevitably align antiparallel which will cost energy to the magnetic system. In order to avoid a singularity in the center of the structure and to minimize the exchange energy, the magnetization tilts out of the plane of the film in a small region forming the vortex core (VC) pointing either up $(p=+1)$ or down $(p=-1)$ termed as the polarity of the vortex core. The combination of circulation and polarity also gives the vortex a handedness (righthanded for $c p=+1$ and left-handed for $c p=-1$ ). Even though the vortex core has a radius of about $5-10 \mathrm{~nm}[36,37]$ only, it plays an important role in the magnetization dynamics of such structures and consequently has been intensively studied in the last decade.

The vortex element has four energetically equivalent ground states (Fig. 2.3), corresponding to left or right circularities and up or down polarities. These magnetic states are highly stable against thermal fluctuations. A high static magnetic field of 
about 0.5 Tesla is required to reverse the polarity. Additionally, due to the dipole free configuration, these structures prevent cross talk among the adjacent vortices and thus have high magnetic stability and can be placed closer to each other. These characteristics make magnetic vortices a possible candidate for more stable and high density magnetic data storage devices [38, 39]. However, if these micromagnets are to be used in technology, a way must be found to combat the enormous stability to control all four states independently and detect them electrically [40, 41] as well. A magnetic vortex exhibits a rich variety of phenomena e.g. it interacts with magnetic fields, transport currents, temperature gradients, samples surfaces and defects. An understanding and the control of vortex dynamics at the mesoscopic scale may make electronic devices based on the control of vortex dynamics possible, as well as the exploration of fundamental physical processes.

\subsubsection{Vortices in an External Static Field}

In absence of any external field, the vortex core is located in the center of the sample. When such a vortex element is suddenly placed in an in-plane external static magnetic field its core is displaced away from the center following a spiral trajectory about its new equilibrium position which depends upon the direction and strength of the external magnetic field. Due to topology, the static displacement of the vortex core is perpendicular to the direction of the external magnetic field and depends upon the chirality but is independent of the vortex core polarity. With increasing external field strength, the vortex core moves away from the center of the magnetic element and is expelled if the magnetic field is strong enough. If we move the vortex core away from its equilibrium position by applying an in-plane external dc magnetic field and then turn the field off abruptly, the vortex core gyrates back to its equilibrium position following a spiral trajectory with its characteristic resonance frequency $[42,43,44]$. The resonance frequency is in first order linear as a function of the aspect ratio (thickness/lateral dimension). Smaller lateral sizes and/or thicker structures will result in a higher resonance frequency. Nevertheless, caution is needed in extrapolating these results. A limit will be reached for too low and too high aspect ratios where a single vortex state is no longer stable. The direction of spiralling motion of the vortex core depends on its polarity and the pitch depends upon the damping parameter of the material. 


\subsection{Vortices in Magnetic Microstructures and their Dynamics}

\subsubsection{Field Induced Vortex Dynamics}

As described in the above section, due to damping the vortex core relaxes to its equilibrium position in absence of external forces. If we supply a certain amount of energy continuously to the system either by an in-plane oscillating magnetic field or current, the vortex core gyrates on a stationary orbit where the damping force is counterbalanced by the supplied energy. This dynamic mode is called gyrotropic mode $[44,45,46]$ the frequency of which is equal to that of the excitation source (field or current). The gyrotropic mode has a well defined eigenfrequency that lies typically in the sub-GHz range for such vortex structures and has a linewidth in the order of tens of MHz. The eigenfrequency is determined entirely by the size and shape of the nanostructure and by the saturation magnetization. In the limit of thin nanostructures this frequency scales with the saturation magnetization and with the thickness of the structure, and inversely with the lateral size of the structure. For small amplitudes of the harmonic excitations, the core moves on an elliptical trajectory in the steady state and the trajectory becomes circular at resonance. This gyrotropic motion can be described similar to that of a forced harmonic oscillator when a parabolic potential is assumed [47]. The approach (see appendix.1) to describe the vortex gyrotropic mode is based on Thiele's formalism [19, 48] of the dynamics of magnetic domains. In his paper, Thiele introduces two integrals (eq. - A1.12) over quadratic functions of the spatial derivatives of the magnetization to simplify calculations of the dynamic properties of magnetic structures. The key assumption is that the magnetic structure does not change its shape when it is moving, thus the time derivative of the magnetization can be written as the spatial derivative of the magnetization times the velocity of the domain wall motion (eq. - A1.6). Then the torque equation of the local magnetization can be written as a force equation for the whole magnetic structure.

$$
\vec{F}_{e f f}+\vec{F}^{g}+\vec{F}^{d}=\vec{F}_{e f f}+\vec{G} \times \vec{v}+\vec{D} \cdot \vec{v}=0
$$

This is the force balance equation $[19,48]$ originally described by Thiele in his paper to describe the dynamics of the domain wall as a rigid particle. For a thin

film geometry the total gyrotropic vector " $\vec{G}$ " is perpendicular to the film plane, i.e. $\vec{G}=G \hat{z}$, if we put the film in (x, y) plane. The gyroconstant is $G=2 \pi p q t_{f} M_{s} / \gamma$ with $t_{f}$ the thickness of the film, the topological charge $q= \pm 1, \pm 2, \ldots$ and the vortex core polarity $p$.

A vortex is approximately described as a particle moving about the system, endowed with an effective mass acted upon by a variety of forces (eq. - 2.8). Clas- 
sically, the vortex has an inter-vortex potential energy giving a Coulomb like force $\left(\vec{F}_{\text {eff }}\right)$, and a gyrotropic force $\left(\vec{F}_{g}\right)$ - behaving as a self-induced Lorentz force, whose direction depends on both topological indices $p$ and $q$. When the local magnetization configuration shows only small deformation, the quasi-particle ansatz by Thiele together with the parabolic potential can be employed to described the dynamics to the vortex core. The energy function is an even function of the vortex core position around the equilibrium position. For small displacements $\vec{X}$ from the equilibrium, it can thus be given by a parabolic function of the vortex core position $X(x, y, 0)$ i.e. $U(\vec{X})=\frac{1}{2} k X^{2}$, where the restoring force constant $k$ is related to the initial susceptibility. If we pump more energy into the magnetic system, the vortex core is excited to gyrate on rather large trajectories and the local magnetization configuration shows distinct deformations [49] which put the assumption of a rigid object into question. Fortunately it turns out that although approaching the limit of the extended Thiele model, it is still applicable for large amplitudes of the excitation by assuming a non-linear potential. Non-linear oscillators change their oscillation frequencies as well as their phases between response and excitation which depends upon the amplitude of the excitation. Investigations of non-linear vortex motion are necessary to achieve a comprehensive picture of vortex motion. Above a certain limit of the excitation power the core switches its orientation which is parametrized by the polarization of the vortex core.

\subsubsection{Current Induced Vortex Dynamics}

It is possible to drive the oscillatory motion of the vortex core directly with an electrical current [50, 51]. Currents flowing through the vortex structure pump energy into the gyrotropic motion of the vortex core via the spin transfer torque effect. The field-induced and current-induced dynamics are very similar and the main difference between these two kinds of excitation is a 90 degree phase shift in the response of the vortex dynamics. Consequently, many results obtained from the field induced excitation can be generalized to the current induced excitation. The excitation of the gyrotropic motion of the vortex core by a spin-polarized current is conventionally described by extending the Thiele equation with a spin torque term as following:

$$
\vec{F}_{e f f}+\vec{G} \times(\vec{v}-\vec{u})+D(\alpha \vec{v}-\beta \vec{u})=0
$$

The STT induced excitation of the vortex core is achieved by electrical currents (charge transport) flowing through the magnetic element and therefore unavoidably accompanied by Oersted fields. Time dependent magnetic fields have been shown to 


\subsection{Vortices in Magnetic Microstructures and their Dynamics}

give rise to the excitation of the vortex state, and thus in the presence of oscillating currents, one expects a combined effect of the STT and the Oe field [52, 53].

\subsubsection{Vortex Core Switching}

The polarity of the vortex core can be switched by means of a strong (around half a Tesla) $[54,55,56]$ external static magnetic field acting in a direction opposite to the vortex core polarization but practical devices can not tolerate such strong fields. This stability has the advantage that magnetic storage of this type would be stable against interfering magnetic fields but also turns into a disadvantage because it is more difficult to reverse the vortex core by static fields and thus to process data. Technological applications require to switch the magnetization states faster and in much more energy efficient ways. An elegant solution has been found to reverse the vortex core polarity much more easily with a mechanism called the dynamic reversal of the vortex core $[57,58,59]$. Previous research work in our group has revealed that instead of an out-of-plane static field, an in-plane oscillating magnetic field with a frequency near to the resonance frequency of the vortex core and with a magnitude of only a few Oe is sufficient to nudge the vortex core out of its equilibrium position and eventually switch the vortex core polarity on a time scale of ns [60,61, 62].

The switching process is shown in detail in Fig. 2.4. The mechanism of the vortex switching by either magnetic field or current is essentially the same in all systems $[55,63,64]$. Initially the vortex core is situated in the center of the vortex structure (Fig. $2.4 \mathrm{a}$ )). When excited with either in plane oscillating magnetic fields or rf currents, the core is pushed away from the equilibrium position and the vortex structure is stimulated to execute a collective spin movement. The moving vortex is followed by a dip with magnetization opposite to that of the vortex core and situated towards the structure center, see Fig. 2.4 (b). When the vortex moves away from the center, its velocity increases and the amplitude of the dip increases as well, see Fig. 2.4 (c). When the amplitude of the out-of-plane dip reaches its maximum a pair of a new vortex and antivortex is created, see Fig. 2.4 (d) - (e). These three objects move following complicated trajectories and the new born vortex tears off his partner antivortex due to topological reasons. The gyroscopic force depends on the total topological charge $q$. Therefore, it produces a clockwise motion for the original vortex and the new born antivortex, while the new born vortex moves in the counterclockwise direction. As a result, the new vortex separates from the vortex-antivortex pair, see Fig. 2.4 (f). The attractive force between the original vortex $(q=1)$ and the antivortex $(q=-1)$ facilitates a binding and subsequent 


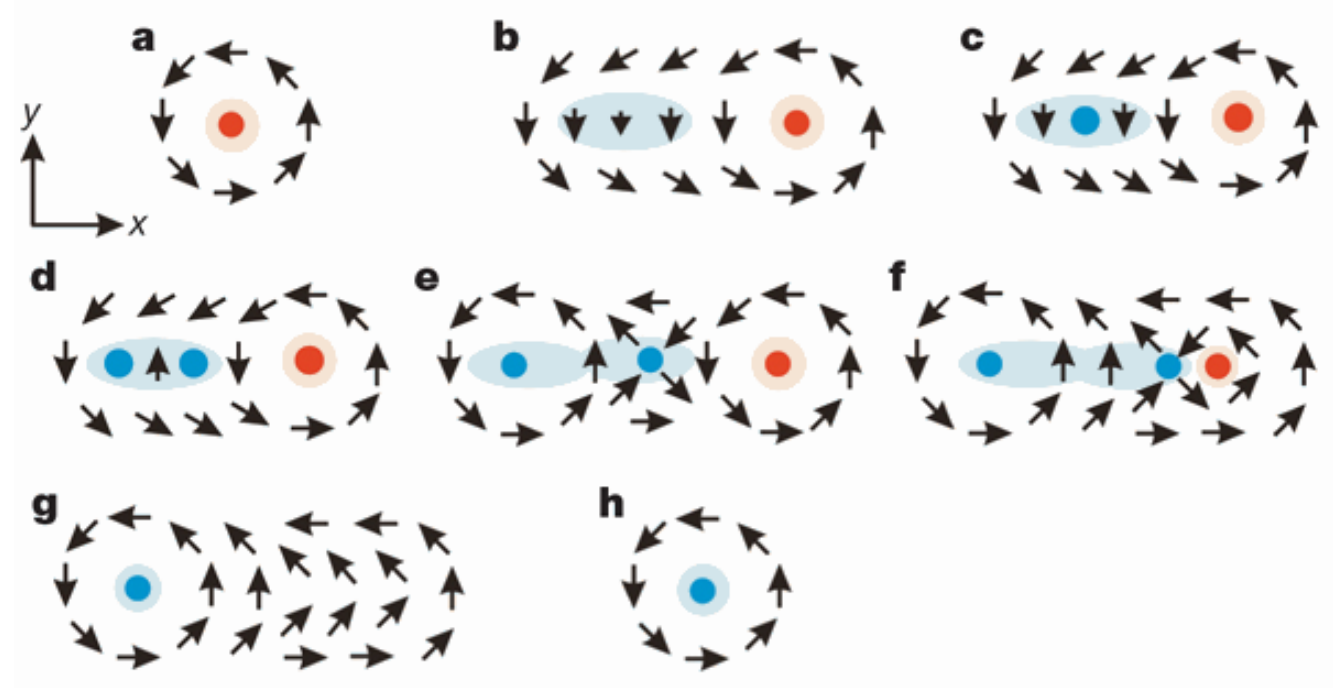

Figure 2.4: Schematic representation of different steps during the switching process of the vortex core polarization. The arrows represent the in-plane magnetization components, and the coloured dot at the centre and the coloured rings around it represent the out-of-plane component (blue, up; red, down). A detailed description is given in the text and in reference [63].

annihilation of the vortex-antivortex pair, see Fig. 2.4 (g), followed by an emission of spin waves $[65,66]$. This results in a new vortex with polarity opposite to that of the original vortex core, see Fig. 2.4 (h). A threshold exists for the field amplitude above which uncontrollable switching processes occur. This threshold will differ from the type of in-plane excitation e.g. pulse [67, 68], burst [63], or continuous excitation that is applied to the structure, and at least is still a factor of about 100 lower than the switching threshold with a static perpendicular field.

\subsubsection{Polarization Selective Switching}

In-plane uniaxial excitations, like linear oscillating fields, do not break the symmetry of mirror-symmetric structures such as squares or disks, and would result in symmetric dynamics for vortices of opposite polarities. Therefore, the resulting switching process is also symmetric - the excitations simply toggle the polarity back and forth and thus the final state depends on both the amplitude and the duration of excitation. In contrast to the linear excitation, which excites the vortex structure irrespective of its polarity, a co-rotating in-plane magnetic field or current couples 


\subsection{Vortices in Magnetic Microstructures and their Dynamics}

differently to the two polarities of the vortex core and hence leads to an asymmetric switching behaviour [61, 69, 70, 71, 72].

The clockwise (CW) and counterclockwise (CCW) circular-rotational motions of a magnetic vortex core in a soft magnetic circular nanodot are the elementary eigenmodes existing in the gyrotropic motion with respect to the corresponding CW and CCW circular-rotational-field eigenbasis. The oppositely rotating eigenmodes show an asymmetric resonance behaviour, i.e., for the up - core orientation the CCW eigenmode shows a strong resonance at the frequency equal to the vortex eigenfrequency, but the other $\mathrm{CW}$ eigenmode shows no resonance. This asymmetric resonance effect is reversed by changing the vortex core polarization. The orbital radius amplitudes and phases of the two circular eigenmodes vary with the polarization and chirality of the given vortex state as well as the frequency. The overall linear-regime steady-state vortex gyrotropic motions driven by arbitrarily polarized oscillating in-plane magnetic fields in the linear regime can be clearly understood by considering it to be the superposition of CW and CCW circular rotational eigenmodes and also considering their asymmetric resonance behaviour.

$$
\begin{gathered}
H_{c c w}=\frac{1}{2} H_{0}\left[\cos \left(\omega_{H} t\right) \hat{x}+\sin \left(\omega_{H} t\right) \hat{y}\right] \\
H_{c w}=\frac{1}{2} H_{0}\left[-\cos \left(\omega_{H} t\right) \hat{x}+\sin \left(\omega_{H} t\right) \hat{y}\right]
\end{gathered}
$$

If the natural gyration sense of the vortex core matches with that of the rotating magnetic field, the vortex core is accelerated to a larger orbit and if the excitation amplitude is sufficient, the core polarization switches and now the vortex core decelerates and spirals back towards the equilibrium position and only a small clockwise gyration remains. Measurements on squares and circular elements further showed that there is no significant influence of the sample geometry on the selective coupling of the rotating magnetic field with the vortex structure. These observations indicate that the core can be unidirectionally switched using rotating fields: an up vortex and a down vortex can be reversed by, respectively, a CCW and a CW rotating field of sufficient amplitude. When the core polarization has switched, the rotating field can no longer excite the vortex core of the other polarity to the threshold velocity required for switching unless the rotation sense of the field is reversed as well. 


\section{Chapter 2. Theoretical Background}
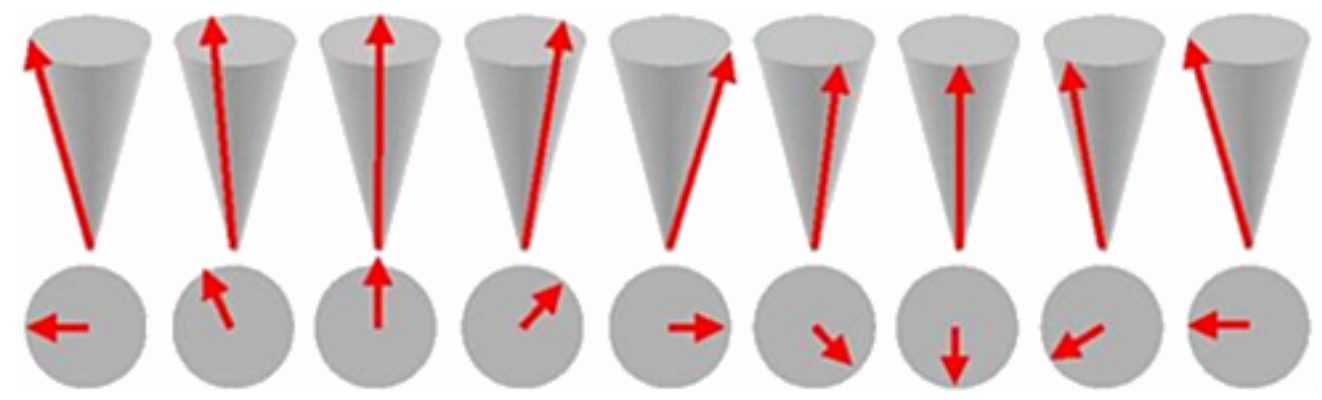

Figure 2.5: Illustration of a spin wave or magnon.

\subsection{Spin Waves}

Spin waves are small amplitude propagating disturbances in the ordering of magnetic materials [73, 74, 75, 76, 77]. In quantum calculations, such collective excitations are treated like particles (quasiparticles) in the wave particle duality limit and the spin wave quanta (magnons) are similar to the quanta of light (photon) or those of acoustic waves (phonons) except that their dispersion relation is quadratic in the wave vector $\vec{k}$.

If we place a ferromagnet in a sufficiently strong external magnetic field all magnetic moments will align parallel to the external field direction. Now the magnetization orientation of individual spins can be diverted from the equilibrium state by various perturbations such as temperature, pressure, magnetic field, spin-polarized current or electromagnetic waves. If we deflect one or a few of the magnetic moments from their equilibrium position the surrounding moments will also feel this perturbation as they are coupled via dipolar and exchange interactions. The deflected spins do not just return into their initial equilibrium position but precess around the direction of the external magnetic field. In this way a collective excitation of magnetic moments forms and a wave of deflected magnetic moments is produced, a so called spin-wave. In a spin wave the magnetic moments at each site precess about their individual equilibrium directions. Since the spins are coupled with one another through exchange and dipolar interactions, spin wave excitations are the eigenmodes of the magnetic system and are characterized by frequency and wavelength. The frequency of a spin wave may depend on the exchange coupling between spins as well as other effective fields caused by for example anisotropies and magnetoelastic effects. These interactions will not only affect the frequency of precession but also the relative phase of precession between spins at neighbouring lattice sites, see Fig. 2.5.

The frequency with which the spins precess is an important property of a spin 
wave. This precession does not last forever, after a certain time the precessing spins cause the atoms they are attached to, to vibrate on their lattice site. The vibration of atoms in a crystalline lattice is perceived as heat. In this way spin waves decay into heat after a certain time. These collective excitations are key features for magnetization dynamics and their control allows for deeper insights into the fundamental processes for magnetization reversal. Exciting spin waves in nanostructures is not just an elegant way to characterize the structure but it also enables a better control over the magnetization reversal processes. It has been observed that magnetization (vortex core) switching is even easier and faster when assisted by spin waves [78, 79, 80]. In ferromagnetic systems such as $\mathrm{Fe}$, Co and $\mathrm{Ni}$ the lowest spin wave frequencies are typically of the order of $10 \mathrm{GHz}$. These are long wavelength excitations and can be studied using FMR and BLS techniques. The lowest mode is the uniform mode, where all spins precess in phase. This uniform mode can be accessed by FMR experiments and is determined by the key properties such as the anisotropy and the saturation magnetization.

Spin waves describe small amplitude deviations of the spins from the equilibrium orientation. If the angle of magnetization precession is small, the spin wave dispersion relation can be obtained as a plane wave solution of the LLG equation if nonlinearity is neglected. For an infinitely extended magnetic crystal, the spin wave dispersion relation is given by the Herring-Kittel formula [73, 76].

$$
\omega(\vec{k})=\gamma\left[\left(H+\frac{2 A}{M_{s}} k^{2}\right)\left(H+4 \pi M_{s} \sin ^{2} \theta_{\vec{k}}+\frac{2 A}{M_{s}} k^{2}\right)\right]^{1 / 2}
$$

where $A$ is the exchange stiffness constant, $H$ is the magnetic field acting parallel to the easy axis, $\vec{k}$ is the wave vector and $\theta_{\vec{k}}$ is the angle between the direction of the wave vector and the magnetization.

The spin wave modes of an infinite ferromagnetic film, magnetized parallel to its surface, were studied by Damon and Eshbach [81]. The dispersion relation for such bulk spin waves is described by equation 2.12, for details see Ref. [82]. They employed the magnetostatic approximation, in which the exchange stiffness parameter $A$ is set to zero. These spin waves have frequencies, which ranges from $\gamma H$ for propagation parallel to both the surface and the magnetization, to $\gamma\left[H\left(H+4 \pi M_{s}\right)\right]^{1 / 2}$ for propagation in plane but perpendicular to the static magnetization. The DamonEshbach (DE) surface spin wave modes exist only when the magnetization is parallel to the surface. These magnetostatic spin waves (modes) have sufficiently long wavelength that exchange may be ignored in their description.

Figure 2.6 shows a typology for different orientations between the in-plane wave 


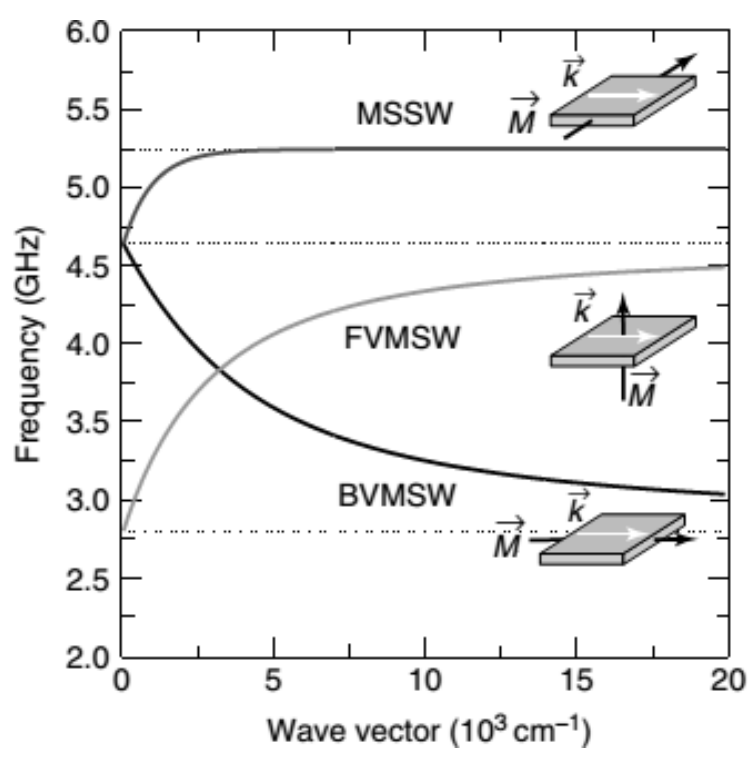

Figure 2.6: Typology of magnetostatic spin-wave modes for different directions of the magnetization, $\vec{M}$, and the in-plane wave vector, $\vec{k}$. MSSW: magnetostatic surface spin waves; FVMSW: forward-volume magnetostatic spin waves; BVMSW: backward-volume magnetostatic spin waves. (Taken from reference [82])

vector $(\vec{k})$ and the magnetization $(\vec{M})$. If $\vec{k}$ and $\vec{M}$ are both in the film plane and if $\vec{k}$ is perpendicular to $\vec{M}$, the so-called magnetostatic surface wave (MSSW) exists. In this DE geometry $(\vec{M} \perp \vec{k})$, the phase velocity $(\omega / k)$ and group velocity $(d \omega / d k)$ have the same sign and thus DE waves are also called forward waves. If $\vec{k}$ and $\vec{M}$ are collinear in the film plane, a mode with negative dispersion, the so-called backward-volume magnetostatic spin-wave (BVMSW) mode exists with the group velocity antiparallel to the wave vector. For the magnetization pointing perpendicular to the film plane, the existing modes are the so-called forward-volume magnetostatic spin-wave (FVMSW) modes. In this case spin wave propagation does not depend on the direction of the in-plane wave vector.

\subsubsection{Spin Waves in Magnetic Vortex Structures}

Besides the low frequency gyrotropic mode (described earlier in this chapter), higher frequency eigenmodes of the vortex structures have also been observed experimentally [44, 83, 84]. Mainly magnon modes of two kinds are excited in this system: radial (symmetrical) and azimuthal ones. The azimuthal mode is caused by the influence of the in-plane field and the radial one by the effective out-of-plane field. 


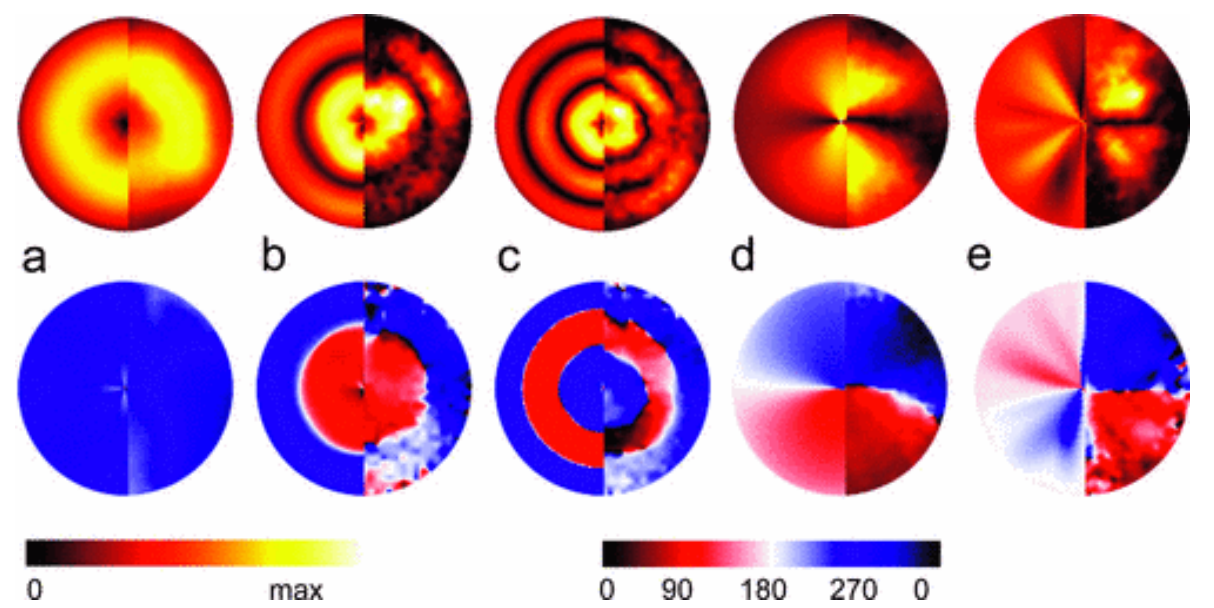

Figure 2.7: Spin waves in magnetic vortex structures. Images of the eigenmodes for a disk with $R=3 \mu \mathrm{m}$ and $d=20 \mathrm{~nm}$. The top row shows the absolute value of the Fourier amplitude at resonance, the bottom row the phase. The modal maps are composed from two half-images: the left from the micromagnetic simulation; the right from the experiment. (a)-(c): Axially symmetric modes showing concentric nodes $(n=1,2,3, m=0)$. (a) $2.80 \mathrm{GHz}$. (b) $3.91 \mathrm{GHz}$. (c) $4.49 \mathrm{GHz}$. (d), (e): These modes have nodal lines going across the image. (d) $2.08 \mathrm{GHz}$, one azimuthal node (1,1). (e) $1.69 \mathrm{GHz}$, two azimuthal nodes (1,2). Notice that across the nodal lines the phase changes by 180. (Taken from reference [88])

The magneto-static spin wave modes $[85,86]$ can be characterized by a radial mode number $n$ (positive integer values starting with 1) and an azimuthal mode number $m$ (integer values), with $n$ and $|2 m|$ being the number of nodes in the radial $\hat{r}$ and azimuthal $\hat{\phi}$ direction. The sign of $m$ denotes the rotation sense of the azimuthal wave [counterclockwise (CCW): $m$ positive, clockwise (CW): $m$ negative]. Recently, such spin wave modes in micron size magnetic disks have been excited by a perpendicularly oriented magentic field pulse and imaged using time resolved magneto-optic Kerr microscopy [87, 88]. Figure 2.7 shows the amplitude (top) and the phase (bottom) at resonance for various eigenmodes where the left-hand side of each image shows the result of micromagnetic calculations and the right hand side is the result obtained from the experiment. One can recognize two types of modes: radial and azimuthal modes. Figure 2.7 (a) - (c) shows three low-lying axially symmetric (radial) modes which are organised into concentric rings with circular nodes. The nonaxially symmetric (azimuthal) modes Fig. 2.7 (d) and (e), however, have one and two diametric nodes, respectively. The modes can be clas- 


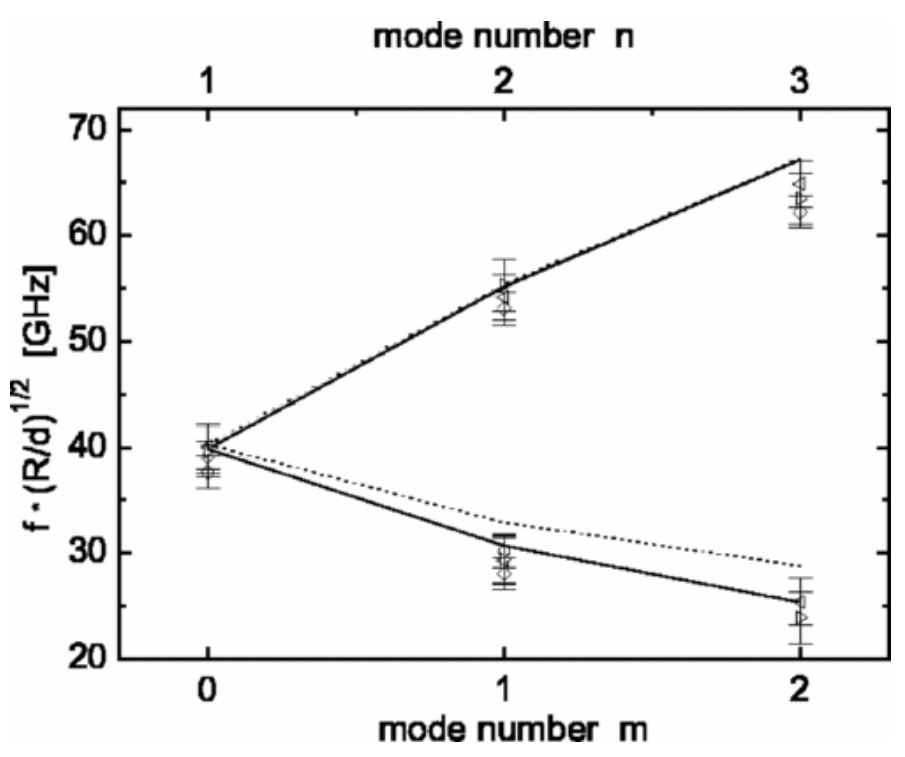

Figure 2.8: Dispersion relation for magnons in a FM disk. The mode frequencies are normalized to the aspect ratio (radius/thickness) as indicated in the figure. (Taken from reference [88])

sified according to the number of nodes in radial and azimuthal directions. The experimental dispersion relations $\mathrm{f}$ vs $(\mathrm{n}, \mathrm{m}=0)$ (radial modes) and $\mathrm{f}$ vs $(\mathrm{n}=1, \mathrm{~m})$ (azimuthal modes) was constructed which is shown in Fig. 2.8. We can notice a positive dispersion (monotonically increasing) for the axially symmetric mode with $m=0$ and a negative dispersion [88] (monotonically decreasing) for the modes $m \neq 0$.

Recently is has been discovered that these higher frequency modes can be used to switch a vortex core at least 20 times faster than was previously the case, i.e. within around 200 picoseconds [89, 90, 91]. The observed effect has the further advantage in addition to its higher switching speed that the vortex core remains almost stationary when switching is performed with spin waves. When switched more slowly with frequencies in the Megahertz range, as was the case with the mechanism discovered a few years ago, on the other hand, it had to be moved far from its equilibrium position. This meant that a storage bit required more space. The new mechanism thus makes further miniaturisation possible if data storage media which operate according to this principle are to be developed in the future. 
Chapter 3

\section{Detection Methods and Micromagnetic Simulations}

A range of available techniques provide many options to experimentalists for the study of magnetic properties at the nanoscale. The ferromagnetic resonance (FMR) technique has been one of the most often used techniques to study magnetization dynamics. However, the frequency of the vortex motion is generally too low (sub$\mathrm{GHz}$ ) to be detected by means of standard ferromagnetic resonance techniques. The dynamic properties of magnetic vortices can also be probed using a vector network analyser in the microwave reflection mode [92], giving quantitative information but no spatial information. This chapter includes some of the techniques to visualize the vortex state and its dynamics and also to investigate it electrically.

\subsection{Imaging Methods}

Although, the structure of the magnetic vortex has been predicted long before in theory, the experimental observations have been possible only recently. First observations of the magnetic vortex core are done in square and circular dots of Permalloy $[15,16]$. In these experiments, the authors used magnetic force microscopy (MFM) to detect the core of the magnetic vortex. As the core has a perpendicular magnetization and the magnetization vector in regions away from the center is parallel to the plane, the force between the cantilever tip and the surface of the core is different from the force between the cantilever tip and the surface of an out-of-core region. Thus the vortex core is imaged by mapping the force all over the disk sample.

Many spectroscopic techniques are used to investigate magnetic properties. These techniques involve the use of electrons and photons which are easily available probes to investigate matter. In a typical spectroscopic experiment, an electron or a photon has a well defined state (energy, direction of propagation, polarization etc.). Today, several such techniques exist to image the magnetization with high spa- 
tial resolution, like Scanning Tunneling Microscopy (STM), Scanning Electron Microscopy with Polarization Analysis (SEMPA) and Lorentz Transmission Electron Microscopy (TEM) etc. These techniques have already been successfully deployed to image small magnetization features like domains and vortex cores. Typically, these techniques do not provide temporal information and can thus only be used to explore static configurations but time-resolved techniques exist as well.

Great progress has been made recently with the possibility to observe high frequency dynamic properties of magnetic dots in the vortex state by Brillouin Light Scattering (BLS) of spin waves, time resolved Kerr microscopy, phase sensitive Fourier transformation techniques and X-ray imaging techniques. Three main ingredients are needed for the imaging of time resolved magnetization dynamics: a pulsed light source, a synchronized fast magnetic field pulse (or alternating magnetic field), and a variable time delay. Only for a few time-resolved imaging techniques the aforementioned ingredients have been implemented successfully. Some of the most popular time or frequency resolved techniques are briefly addressed in the following section.

Brillouin Light Scattering (BLS) technique can be employed to study magentization dynamics in patterned structures such as arrays of ferromagnetic wires and arrays of magnetic dots with vortex structures [93]. This technique is a spectroscopic method to investigate inelastic excitations with frequencies in the $\mathrm{GHz}$ range [94]. Basically, a light beam is sent from a frequency-stabilized laser to focus it onto the sample using an objective lens. The light scattered from the sample (elastic and inelastic contributions) is then collected and sent through a spatial filter to suppress background noise before it enters a Fabry-Perot interferometer. Vortex dynamics observed by BLS have relatively high frequencies and can be interpreted as quantized spin waves in restricted geometries. BLS, however, does not permit the detection of eigen-frequencies in sub-GHz range.

The excitation and relaxation processes in ferromagnetic structures can also be imaged by means of Time-Resolved Magneto-Optical-Kerr (TR-MOKE) microscopy $[87,88]$, in which the combined picosecond temporal and submicrometer spatial resolutions allow to study the time dependence of magnetic excitations and to acquire magnetic maps of the sample. The optical measurements performed with this technique have been performed using a femtosecond or picosecond laser. The polar or transverse Kerr rotation is monitored by a polarizing beam-splitter and by using a differential diode detection scheme, which measures the linear MOKE signal. The lateral resolution is limited by optical diffraction effects and may reach 


\subsection{Electrical Detection}

values of about $300 \mathrm{~nm}$. The time resolution is given by the width of the laser pulses and the jitter of the synchronization. Solid state lasers and fast laser diodes deliver pulses in the 10 ps range. Shorter pulses of the order of 50 - $100 \mathrm{fs}$ are obtained when using a mode-locked Ti-sapphire oscillator. Although the time resolution is amply sufficient to resolve the gyrotropic mode in structures with micron and sub-micron size, the lateral resolution of the MOKE techniques is limited by the wavelength of the used light (typically $\lambda>200 \mathrm{~nm}$ ) which is insufficient to study the details of the gyrotropic motion or to image a vortex core (VC) of only $20 \mathrm{~nm}$ in diameter.

Time-resolved x-ray microscopy, on the other hand, is capable of combining a good time resolution with the necessary lateral resolution. A lateral resolution of about 20 - $40 \mathrm{~nm}$ is achieved using X-ray based imaging techniques. The time resolution is given by the width of the photon flashes and is about $70-100$ ps. Scanning Transmission X-ray Microsopy (STXM) [63] techniques that utilize Xray Magnetic Circular Dichroism (XMCD) [95] to obtain the magnetic contrast are emerging as one of the premier probes. The vortex core dynamics induced by a pulsed magnetic field has also recently been detected in STXM [68, 91]. We have utilized this technique in our experimental work, the detailed description of which will be presented in following chapters.

\subsection{Electrical Detection}

Electrical detection of the vortex state is a useful tool not only for understanding the underlying physics related to vortex dynamics but for future technological applications as well. With this aim, we studied two ways to electrically detect the polarity and/or circulation of the vortex state in soft ferromagnetic elements of micron size. In spintronics research the ability to generate detectable electrical voltages due to time dependent magnetic textures has been addressed recently [96, 97, 98]. Among the methods discussed in the literature are effects due to spin pumping [99, 100], the anisotropic magnetoresistance (AMR) effect [96] and, more recently, due to the so called spin motive force (SMF). We will explore AMR and SMF effects in vortex structures to understand its origin and how to use these phenomenon for the detection of the vortex state.

\subsubsection{AMR in Vortex Structures}

When a current flows through a ferromagnetic conductor the charge transport is affected by the global spin alignment in the material and so is the corresponding 


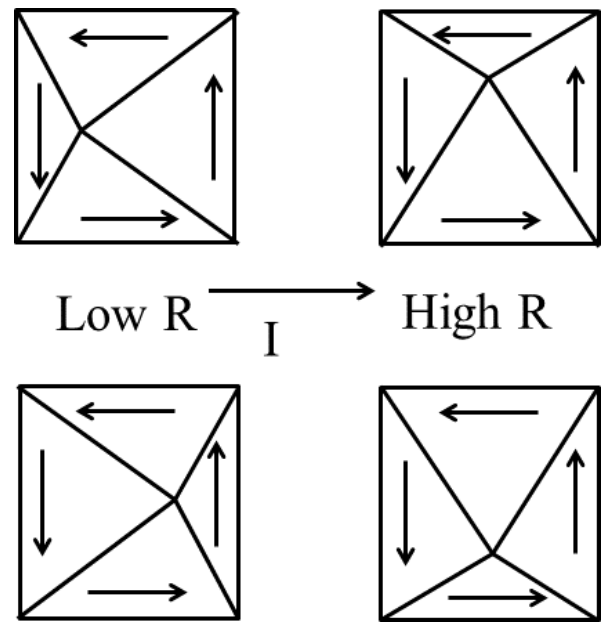

Figure 3.1: The resistance states of the vortex element when the vortex core is displaced in different directions with respect to the current flow direction.

electrical resistivity. This effect is called the Anisotropic Magnetoresistance (AMR) effect [101, 102, 103, 104, 105]. The microscopic origin of the AMR lies in the fixed spin orientation in ferromagnetic materials which breaks the otherwise spherical symmetry of the electrons' atomic orbitals around the magnetization axis. Consequently the scattering cross section of the electrons, which is defined by their orbitals, is different for current parallel and perpendicular to the magnetization direction and so is the corresponding electrical resistivity. The resistivity collinear to the magnetization is usually higher than that for perpendicular alignment to the magnetization e.g in the case of Permalloy.

In this section, we present a conceptually simple technique to determine the vortex state electrically by exploiting the Anisotropic Magnetoresistance effect where measurements can be made with straightforward four point transport measurements. The magnetic response measured with such measurements is basically a bulk response and thus we expect this method to be free from many of the surfacerelated problems of the aforementioned imaging techniques. When an rf current $\left[I=I_{0} \cos (\omega t)\right]$ flows through the vortex structure (or magnetic texture), as a result of the spin-transfer-torque exerted on the local magnetic moments, the vortex core starts to gyrate around its equilibrium position. Consequently, the magnetization configuration of the sample changes periodically with the position of the vortex core (see Fig. 3.1). Since the resistance of the ferromagnetic sample depends upon the contribution of the sample magnetization along (or perpendicular to) the current flow direction, the gyrotropic motion of the vortex core is followed 


\subsection{Electrical Detection}

by a periodic change in the resistance of the magnetic sample. As the vortex core moves symmetrically around the center of the magnetic element, its resistance $\left[R=R_{0}+\Delta R \cos (2 \omega t+\phi)\right]$ changes with twice the frequency of the excitation current $[106,107]$. This can simply be explained by the sketch shown in Fig. 3.1. If we move the vortex core along the current flow direction, the effective magnetization of the sample is perpendicular to the direction of the current flow. Thus, these two states have low resistance. In the other case, when the vortex core is displaced perpendicular to the current flow direction, the effective magnetization is along the current flow and thus these two states have high resistance. Therefore, over a period of gyration, the resistance of the sample goes through high and low resistance states twice which means the periodicity of the resistance change is twice to that of the excitation current. This periodic change in resistance combined with the excitation current gives rise to a homodyne voltage signal (signify that the reference mixing signal is derived from the same source as the original signal which has the advantage of being insensitive to the fluctuations in the frequency of the original signal) which can be understood simply by following Ohm's law:

$$
\begin{aligned}
V & =I \times R \\
& =I \cos (\omega t) \times\left[R_{0}+\Delta R \cos (2 \omega t+\phi)\right] \\
& =I R_{0} \cos (\omega t)+\frac{I \Delta R}{2}\{\cos (\omega t+\phi)+\cos (3 \omega t+\phi)\}
\end{aligned}
$$

This homodyne signal can be measured e.g. in an oscilloscope and the vortex dynamics can be investigated. However, we are interested in the rectification of this homodyne voltage signal to measure the dc response because in electronic circuits it is easy to deal with de signals compared to the rf signals which is more significant for device applications. To obtain the rectified voltage signal, we need to break the symmetry of the magnetic configuration to be able to generate a component in the oscillating resistance which is changing with the frequency of the excitation current. This can be achieved either by creating a defect in the magnetic element or by moving the vortex core away from the center of the magnetic element by applying an external static magnetic field. In this case the resistance can be expressed as:

$$
R=R_{0}+\Delta R \sum_{n \neq 0} A_{n} \cos (n \omega t+\phi)
$$

Here $A_{n}^{\prime} s$ are constant coefficients. This means that the oscillating resistance has a component at the excitation frequency $\left[\Delta R A_{1} \cos (\omega t+\phi)\right]$ which mixes with the 
rf current and generate a dc voltage signal as following:

$$
\begin{aligned}
V & =I R \\
& =V_{0}\left[B_{1} \cos (\phi)+\sum_{n \neq 0} B_{n} \cos (n \omega t+\phi]\right. \\
& =V_{d c}+V_{a c}
\end{aligned}
$$

Here $B_{n}^{\prime} s$ are the constant coefficients. The rectified component of this voltage signal $\left(V_{d c}=B_{1} \cos (\phi)\right)$ can simply be measured e.g. in a nanovoltmeter. Here we notice that the rectified signal is accompanied by the phase factor $\cos (\phi)$. As described earlier, there is a phase difference of 180 degrees for the two polarities of the vortex core and thus we expect a sign reversal of the rectified signal for the two polarities while keeping the other parameters unchanged.

\subsubsection{Micromagnetic Simulations (AMR)}

Micromagnetic simulations have proven to be a useful tool allowing us to predict the magnetization dynamics in magnetic nanostructures and to follow dynamic processes occurring on time-scales of nanoseconds or less. Currently, a number of packages, with their strengths and weaknesses, are available for solving the LLG equation which describes the time evolution of the magnetization under several forces as explained in Chapter-1. To solve the complex LLG equation, discretization in both space and time is necessary. The spatial discretization needs to be fine, since features as small as domain walls and the vortex core need to be resolved. Consequently, a large number of computational cells are often needed. The temporal discretization also needs to be very small compared to the time scale of the excitation modes, so a very large number of time steps is also usually needed. There we should choose a simulation package that can fulfill our requirements and is more efficient. In this research work, simulations are performed with the recently developed open source code MuMax, based on a finite difference method including the adiabatic as well as the non-adiabatic spin-transfer-torque and the Oersted field arising from the current distribution in the sample under investigation, where a spatially homogeneous current distribution is considered.

For the micromagnetic modelling we use a device geometry similar to that used in our experiments - a square of edge length $1.6 \mu \mathrm{m}$ and $50 \mathrm{~nm}$ thickness. In our work we investigate the soft ferromagnet Permalloy (an alloy with about $81 \% \mathrm{Ni}$ and $19 \% \mathrm{Fe}$ ) which has a high saturation magnetization and a low coercivity. It is widely used, e.g. in magnetic sensors. Therefore, the magnetic parameters for mi- 


\subsection{Electrical Detection}

cromagnetic simulations are chosen to replicate Permalloy as following: saturation magnetization $M_{s}=800 \times 10^{3} \mathrm{~A} / \mathrm{m}$, damping constant $\alpha=0.006$ and exchange parameter $A=1.3 \times 10^{-11} \mathrm{~J} / \mathrm{m}$. To study the dynamics of the vortex core which has a size of the order of $10-20 \mathrm{~nm}$, a cell size of $3.125 \mathrm{~nm}$ (less than the size of the exchange length of Permalloy $\sim 5 \mathrm{~nm}$ ) in plane and only one cell in the out of plane dimension was used. Thus, the ferromagnetic particle is subdivided into smaller computational cells and the magnetization is assumed to be uniform within each cell. In many situations, the exact formulation of the damping does not matter. Firstly, static properties like magnetic ground states and hysteresis behaviour do not depend on damping. As long as there is some mechanism for the system to evolve towards the lowest energy state, such properties are calculated correctly. In such simulations, an unphysically high damping is commonly used to speed up the relaxation processes. Secondly, many dynamic processes, like vortex core switching, are too fast for the damping to have a large effect on the relevant time scales. Even when the damping does play a significant role, like during the relaxation of the vortex gyrotropic mode, scaling the damping parameter $\alpha$ is usually sufficient to fit the experimental observations.

Initially to obtain the ground state configuration, the damping constant is set to be 1 to assure rapid convergence. The magnetization distribution is simulated for different applied in-plane static magnetic fields as per our requirements. Simulations shows that for the chosen material (Permalloy) and geometry a single vortex state is energetically favorable. The vortex structure is excited with an rf current to make the vortex core to gyrate in a stable orbit around its equilibrium position in a particular applied field. Now eight phases per period for the magnetization distribution in the sample are stored for several periods of oscillations. In order to calculate the dc voltage caused by the anisotropic magneto resistance effect from the simulated magnetization configurations in a square, we make the following simplifying assumption: the AMR effect changes the resistance only slightly (on the order of a few percent) and therefore we assume that the electric current does not change due to the magnetization configuration. Especially for the square sample we assume that the current is homogeneously flowing in one direction, the x-direction. The resistance in a single cell, indexed by $i, j$ is then given by:

$$
R_{i, j}=R_{0 ; i, j}\left(1+\delta\left(\vec{m}_{i, j} \cdot \vec{I}_{i, j}\right)^{2}\right)=R_{0 ; i, j}\left(1+\delta m_{x ; i, j}^{2}\right)
$$

where $R_{0 ; i, j}$ is the resistance of the cell without taking AMR into account, $\delta$ is the relative resistance change, $I$ the current through the cell and $\vec{m}_{i, j}$ the normalized magnetization vector in the cell. When the relative change in resistance is small 


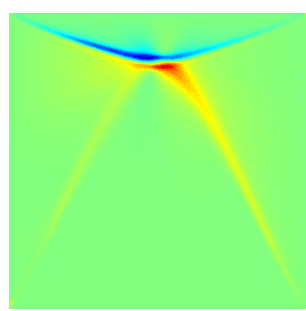

(a) $c=+1$

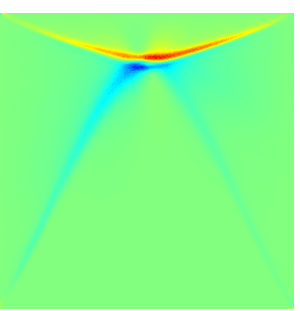

(b) $c=+1$

$p=-1$

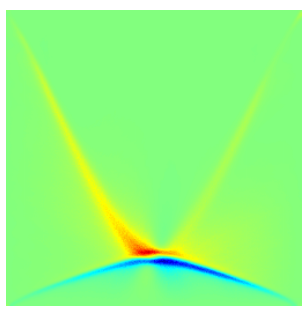

(c) $c=-1$

$p=-1$

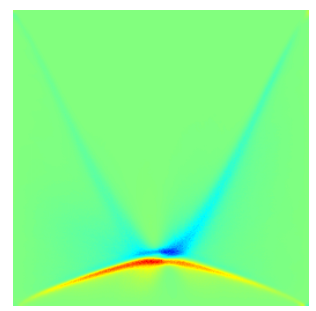

(d) $c=-1$

$p=+1$

Figure 3.2: Micromagnetic simulation results for the AMR contribution to the DC homodyne signal in a square element. Simulations are performed for the four possible configurations of polarity $(p)$ and chirality $(c)$ of the vortex structure.

$\delta<<1$ then we can use a Taylor expansion to easily calculate the total resistance.

$$
R \approx R_{0}+\delta \sum_{i, j} m_{x ; i, j}^{2}
$$

where $R_{0}$ is the total resistance of the element without taking AMR into account. An ac-current that excites the magnetization to periodic precession through spin transfer torques therefore leads to a periodically changing resistance and causes a measurable change in the voltage across the element. The voltage $V=R \cdot I$ consists of a dc part due to rectification with the ac-current:

$$
V_{d c}=V_{\omega=0}=\frac{1}{2} R_{\omega} \cdot I_{\omega}
$$

where $R_{\omega}$ and $I_{\omega}$ are the frequency components of the resistance and the current respectively. From equation 3.5 and 3.6 we can see that the magnetization dynamics contributes to $V_{d c}$ only when $m_{x ; i, j}^{2}$ oscillates with the driving frequency $\omega$. For small perturbations $d \varphi$ at frequency $\omega$ of the equilibrium magnetization lying in the plane of the element this is maximum when the oscillations are around the equilibrium magnetization angle $\varphi$ of $45^{\circ}$ to the current.

$$
m_{x} \propto \sin (\varphi) \Rightarrow \frac{d \sin (\varphi)^{2}}{d \varphi}=\sin (2 \varphi)
$$

The above strategy is used in each cell and the resulting voltage signal is calculated. Figure 3.2 shows the spatially resolved contribution to the rectified AMR signal all over the sample for a bias field of $10 \mathrm{mT}$, an rf current of amplitude 10 $\mathrm{mA}$ and an excitation frequency of $220 \mathrm{MHz}$. The red region shows the positive signal, the blue region shows the negative signal and the green region zero signal. 


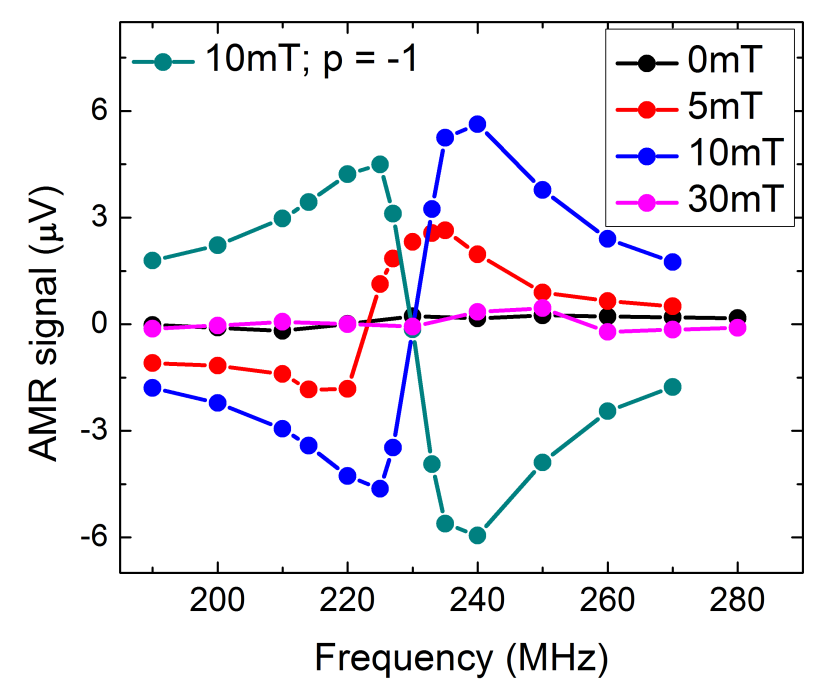

Figure 3.3: Micromagnetic simulations of the rectified AMR signal for various bias fields obtained on the square shaped sample. The sign of the AMR signal changes when the vortex core polarity is reversed as shown for the case of $10 \mathrm{mT}$, where both cases $p=+1$ and $p=-1$ are shown. We do not observe any AMR signal for zero magnetic field due to symmetry reasons and for fields (30 $\mathrm{mT}$ ) large enough to expel the VC from the sample.

Here we notice that the main contribution is concentrated in the region where the vortex core gyrates and the magnetization direction changes periodically following the vortex gyrotropic motion. Also note that there is a contribution in the lines connecting the vortex core with the four corners of the square where the average magnetization direction can be assumed to be at 45 degrees to the current flow direction. In these regions the magnetization direction changes periodically which leads to a periodic change in resistance of the sample. Now we sum up the signal all over the sample which gives us the total rectified signal for the specific parameters (bias field, excitation frequency and amplitude). This signal is calculated for several frequencies of rf current and bias fields. Fig. 3.3 shows resonance curves for the AMR signal at different bias fields. For $0 \mathrm{mT}$ external field there is no resonance line as expected due to symmetry reasons as explained earlier and as seen directly in the spatial contribution images. For $0 \mathrm{mT}$ bias field the vortex core gyrates in the center of the square and in this case the positive (red) and negative (blue) contributions are equal which will add up to zero for the whole sample. For an external bias of 5 and $10 \mathrm{mT}$ there are resonance curves which show a large signal around a 
frequency of $230 \mathrm{MHz}$. For a bias field of $30 \mathrm{mT}$, the magnetic element is not in the vortex state anymore and therefore the AMR signal vanishes. If the polarity of the vortex core is reversed, the vortex core gyrates in the opposite sense and therefore there is a phase $(\phi)$ change of 180 degrees which results in a change of the sign of the AMR signal, following equation-3.3. As we can see in Fig. 3.2 (b), if we change the polarity of the vortex core, the positive (red) and negative (blue) contributions exchange as compare to Fig. 3.2 (a). Therefore the total rectified signal will also change sign which is shown in Fig. 3.3 for a bias field of $10 \mathrm{mT}$ where the shape of the resonance line is reversed for the two polarities of the vortex core. Additionally, if we reverse the circulation of the vortex structure or the direction of the bias field, the vortex core will gyrate near the opposite edge of the square element due to topological reasons. In this case, the positive (red) and negative (blue) regions will expand or shrink as can be visualized by comparing the spatial contributions in Fig. 3.2 (b) and Fig. 3.2 (c). In this case also we will get a sign reversal in the total rectified voltage signals for the two circulations of the vortex structure.

\subsubsection{Spin Motive Force}

Another technique to detect the vortex state electrically is utilizing the phenomenon of Spin Motive Force (SMF) [108, 109], which reflects the conversion of the magnetic energy of a ferromagnetic system into the electrical energy of the conduction electrons via their mutual exchange interaction. When the spin quantization axis of conduction electrons flowing in a ferromagnetic sample does not coincide with the spin quantization axis of local moments in a magnetic texture (e.g. a domain wall or a vortex structure), the interaction of the spin polarized current with the texture can cause e.g. domain wall motion. This effect is known as spin transfer torque as described earlier. Onsager's reciprocity relations dictate that the inverse effect must exist as well. Thus, a moving magnetic texture must lead to an induced voltage (or current) [110, 111], referred to as spin motive force. In a ferromagnet the spin of conduction electrons couples to the local magnetization via the exchange interaction which tends to align the spins parallel to each other. In a moving magnetic texture, the conduction electrons are affected by a spin dependent force due to this interaction. It drives majority-spin and minority-spin electrons in mutually opposite directions and produces a spin current which is followed by a charge current as a ferromagnet has unequal number of majority and minority spin electrons. Thus, the origin of this phenomenon is a misalignment between the conduction electrons spin and the local magnetization. Therefore, SMF can occur in magnetic systems 


\subsection{Electrical Detection}

with strongly non collinear magnetic textures such as magnetic domain walls or magnetic vortices and is given by (for detailed description see Appendix-2) [109]

$$
\left\langle f_{i}\right\rangle_{\uparrow \downarrow}= \pm \frac{\hbar}{2}\left(\boldsymbol{m} \times \partial_{t} \boldsymbol{m}\right) \cdot \nabla_{i} \boldsymbol{m}
$$

From Eq. (3.8), one expects a large SMF signal when fast dynamics is induced on the magnetic structure. It has already been shown theoretically and experimentally that the diameter of the vortex core is of the order of $10 \mathrm{~nm}$ and the velocity of the core dynamics is in the range of $100 \mathrm{~m} / \mathrm{s}$. Furthermore, switching occurs on a time scale of picoseconds. These conditions give us a possibility of observing a large spin motive force signal in vortex structures. On the basis of numerical work, a continuous ac spin motive force is predicted to accompany the gyrotropic motion of a magnetic vortex core and has also been detected in nanoscale magnetic elements recently [110, 111, 112].

Here we summarize how to calculate the spin-motive force. First, we have to know the magnetization dynamics by solving the LLG equation. Then, the spin dependent force acting upon an electron at each time and space can be calculated by Eq. (3.8). The spin-motive force can be obtained by the spatial difference of the electric potential, which is given by the Poisson equation $\frac{P}{-e} \nabla \cdot\langle f\rangle_{\uparrow}=\Delta V$. Here, $e$ and $P$ are the elementary charge and the spin polarization of the conduction electrons, respectively.

\subsubsection{Micromagnetic Simulations (SMF)}

Micromagnetic simulations for the spin motive force signal are performed for a disk of diameter $1.6 \mu \mathrm{m}$ and thickness $50 \mathrm{~nm}$. Other simulation parameters are chosen the same as those for the AMR simulations. The resonance frequency for this geometry is calculated to be $256 \mathrm{MHz}$. We focus on the dynamics of the magnetic vortex core excited with a linear in-plane oscillating magnetic field $\left[h_{r f} \cos (2 \pi f t)\right]$ along the x-direction with an amplitude $h_{r f}(2.5 \mathrm{Oe})$ and a frequency of $255 \mathrm{MHz}$. Simulations are run for a period of 20 ns to let the vortex core gyrate into a stable orbit. In this case, the vortex core starts to gyrate around its equilibrium position on an orbit with a radius of about $140 \mathrm{~nm}$. The magnetization distributions are recorded at a time interval of 1 ps which are used to calculate different product and derivative terms to solve Eq. (3.8) numerically which gives the spin-dependent force acting on the conduction electrons. Thereafter, the local potential is calculated using the Poisson equation as described in the previous section. Thus we obtained 


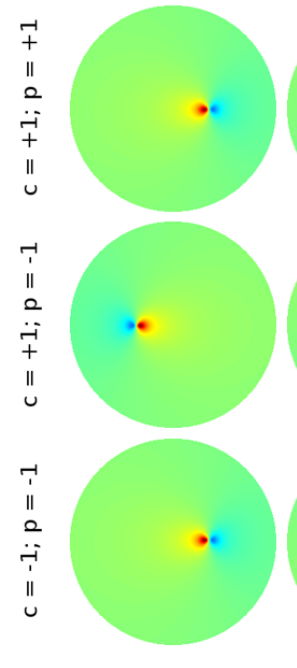

$t=0$
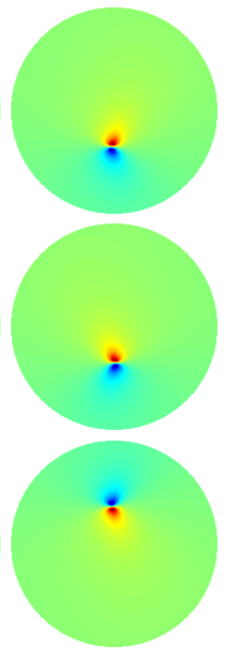

$\mathrm{t}=\mathrm{T} / 4$

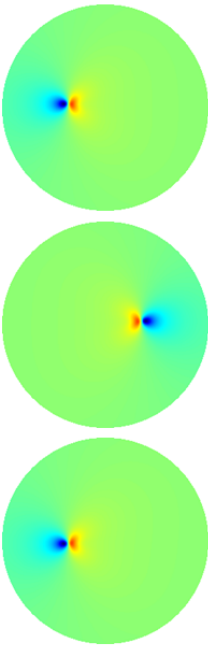

$\mathrm{t}=\mathrm{T} / 2$
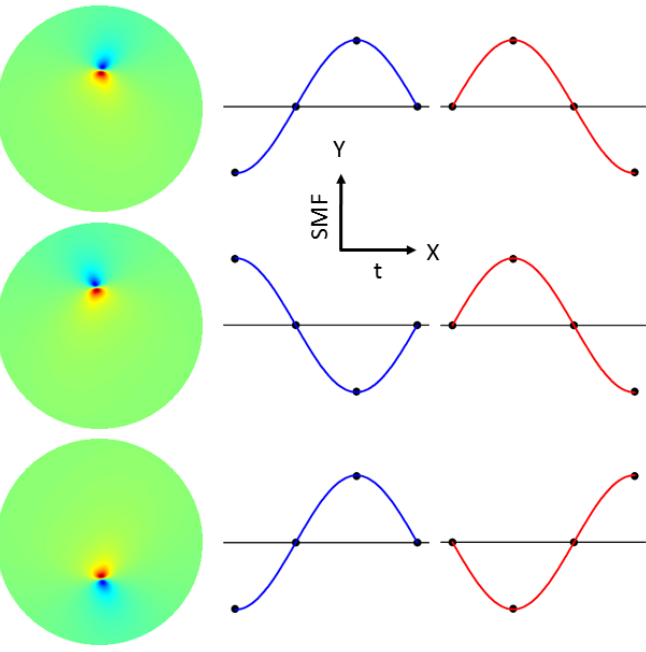

$t=3 T / 4$

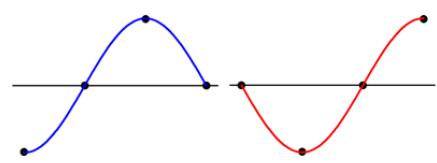

$x$

Figure 3.4: The spatial distribution of the SMF generated voltage signal is shown for three different combinations of circulation $(c)$ and polarization $(p)$. The snapshots are taken at four phases during a complete cycle. Last two columns show the sketch of the expected SMF signal when the signal is measured along the excitation field direction (column-X) and perpendicular to it (column-Y). We can notice that there is a phase change of 180 degree for the two polarities if the signal is measured along the field direction and no phase shift if measured in perpendicular direction. Also there is a phase change of 180 degree for the two circulation directions in both configurations.

the potential distribution all over the magnetic disk due to the SMF acting on the conduction electrons.

Figure 3.4 shows four phases of these SMF generated voltage landscapes for three different combinations of the circulation and polatization of the vortex state. The snapshots are taken at times $\mathrm{t}=0, \mathrm{~T} / 4, \mathrm{~T} / 2$ and $3 \mathrm{~T} / 4$, where $\mathrm{T}$ is the time period. Here we notice that at $t=0$ when the excitation field is maximum, the position of the vortex core is along the field direction but at opposite sides of the centre of the disk for different circulation/polarization of the vortex state. In other words we can say that the phase of the vortex core motion depends upon the handedness $(c p)$ of the vortex state. From these four phases we can draw a sketch of the nature of the SMF signal as shown in the last two columns of Fig. 3.4. Here column-X means the signal along a line collinear with the direction of the excitation field and similarly column-Y means the signal along a line perpendicular 


\subsection{Electrical Detection}

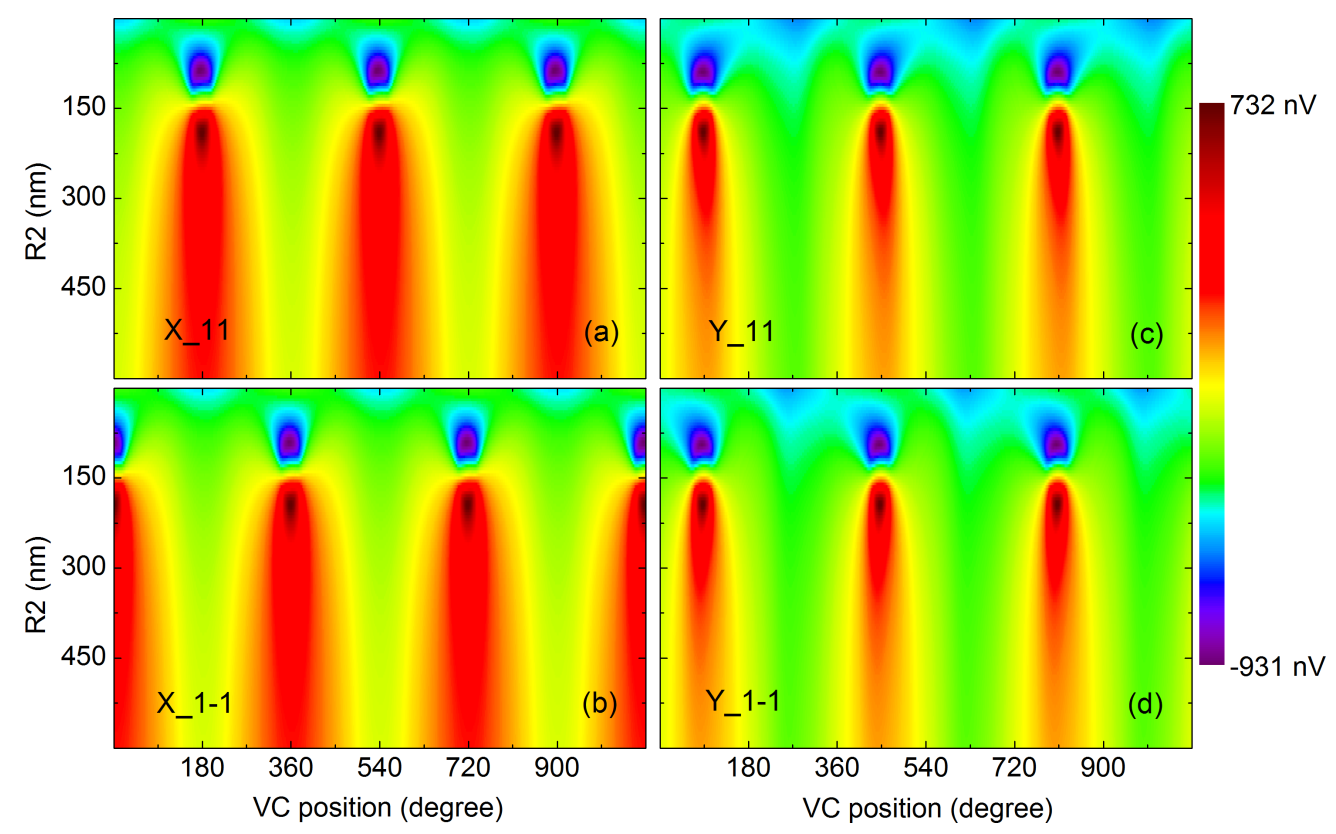

Figure 3.5: The SMF signals derived from micromagnetic simulations are shown for two polarities and for two directions of the probing contacts. Here position of one contact (R1) is fixed at $600 \mathrm{~nm}$ and the SMF signal is calculated by changing the position of the other contact (R2). The excitation field is considerd to be in $x$-direction. The images are marked with $X_{-}$cp or $Y_{-}$cp, where $X$ and $Y$ indicates, in which direction the probing contacts are considered. Here we can see that the SMF signal has a phase shift of 180 degree for the two polarities when the signal is probed in $x$-direction and no phase shift in other case.

to the field direction. The sketches in column-X indicates that if we place voltage probes along the field direction, the voltage signal will have a phase shift of 180 degree for different handedness and if we place the probes perpendicular to the field direction there is a phase shift of 180 degree in the SMF signal only if the circulation direction is reversed.

Next we calculated the periodic SMF signal while varying the position of one probe contact (R2) and the position of the other contact (R1) remains near to the circumference of the disk. This can be achieved by recording a number of magnetization distributions over a complete gyration of the vortex core, which will be a time consuming process. Alternatively, we used one of the potential distribution shown in Fig. 3.4 and calculated the SMF signal by rotating the position of the probe contact i.e. in the rest frame of the vortex core. Figure 3.5 shows spectra of the simulated SMF signal for the two polarities of the vortex core when the 
probe contacts are placed parallel $(\mathrm{X})$ and perpendicular $(\mathrm{Y})$ to the excitation field direction $(x)$. Here we can see a phase shift of 180 degree for the two polarities of the vortex core for the parallel case and no phase shift for the perpendicular case.

\subsection{Exchange Bias}

The forementioned micromagnetic simulation results show, for example, that the sign of the AMR signal depends upon the handedness $(c p)$ of the vortex structure. Thus two out of the four possible configurations $(c$ and $p$ ) of the vortex state will generate a positive AMR signal and the other two will generate a negative signal for the same excitation parameters and bias field. Thus we need to distinguish all four states independently which can be done by utilizing in-plane exchange bias in such vortex structures $[113,114]$. It has been shown that it is possible to imprint well defined nonuniform magnetization states, such as a magnetic vortex, into the antiferromagnet by bringing the two in contact and treating the stack with heat and magnetic fields. The exchange bias can be represented as an additional position dependent magnetic field the direction of which is defined by the magnetization direction in the ferromagnetic layer that is imprinted into the antiferromagnet. Micromagnetic simulations carried out by Heinonen et. al. [115] have shown that the resonance frequency of the vortex core increases with the magnitude of exchange bias considering that the exchange bias field follows the direction of the magnetization in the ferromagnetic layer. We have performed similar micromagnetic simulations and found that if we keep the direction of the exchange bias and change the circulation of the vortex structure, there is a splitting of the resonance frequencies for the two circulations of the vortex state irrespective of its polarity. When the direction of the exchange bias field follows the magnetization direction of the vortex structure, the effective saturation magnetization of the ferromagnetic layer increases and thus the resonance frequency is higher $(202 \mathrm{MHz})$ and it is lower (119 $\mathrm{MHz}$ ) if the exchange bias field direction is opposite to that of the magnetization in the vortex structure compared to the one in absence of exchange bias (161 MHz). Thus exploiting exchange bias in vortex structures we expect to obtain positive and negative AMR signals for the two polarities of the vortex core at higher frequency in the case of one circulation and similar signals at lower frequency for the other circulation. We note that the circulation can be switched selectively by application of a magnetic field when slight asymmetries in the shape of the vortex state elements are introduced $[116,117]$. 


\section{Sample Preparation}

Sample preparation is a matter of great importance as it is a critical determinant for the success of an experiment and thus the device fabrication requires sophisticated and demanding approaches [118]. A proper experimental model and careful sample preparation is crucial in research and has to be carefully considered to obtain significant and trustworthy results. The fabrication of submicron microelectronic devices requires a wide variety of physical and chemical processes performed on a substrate e.g., silicon and GaAs. In general, the fabrication of the devices measured during this research work was a multistep process utilizing different techniques which will be described in detail throughout this chapter. We will discuss strategies and methodologies of sample preparation, and experimental demands for these processes. Additionally, different types of samples prepared for several other research projects will also be presented.

The University of Regensburg houses all necessary equipment and other necessary utensils required to prepare devices investigated during the course of this thesis work. Patterning of our nanostructured devices is done in a clean room. The supporting infrastructure consists of a water purification system, a pure gas installation, a photo resist coating system (spinner), an UV-exposure system using Hg-lamp, inspection optical microscopes, an ion etching system, a wire bonder, pre/post bake heater sources, developer solution, metal deposition chambers and a sonication bath for substrate cleaning and easy lift off process with hard substrates. The device fabrication steps involved wafer preparation, writing of a specifically designed pattern utilizing Electron-Beam-Lithography (EBL) and/or Optical-Lithography (OPL) followed by metal deposition (sputtering, thermal and e-gun evaporation methods) on the developed samples and a resist stripping step so called lift off process. All must be carried out with a high degree of process control and spatial uniformity. 


\subsection{Methods and Techniques}

\subsubsection{Optical Lithography}

Fundamental of various processes used to fabricate an integrated circuit is the lithography, i.e., the formation of a three-dimensional (3D) relief images on the substrate for subsequent transfer of the pattern to the substrate. The word lithography comes from the Greek words lithos - meaning stones and graphia - meaning to write. It means quite literally writing on stones. In our case, our stones are substrate wafers and our patterns are written with a light sensitive polymer called photoresist. Optical lithography involves a complex photographic process in which electromagnetic waves are incident on a photoresist which dynamically changes during illumination and forms a 3D relief image on the substrate after development in a suitable chemical called developer. In general, the photoresist image has the exact shape of the designed or intended pattern in the plane of the substrate, with vertical walls through the thickness of the resist. Thus, the final resist pattern is binary: parts of the substrate are covered with resist while other parts are completely uncovered. This binary pattern is needed for pattern transfer by several mechanisms e.g. etching, ion implantation, deposition and lift-off technique. A single iteration of lithography is a sequential combination of several steps. The general sequence of steps for a typical optical lithography process is described below.

Substrate Preparation: To facilitate the transmission of X-rays through the magnetic element, the samples are grown on a high quality thin $(100 \mathrm{~nm})$ silicon nitride $\left(S_{3} N_{4}\right)$ membrane window (lateral dimension of about $50 \mu \mathrm{m} \times 50 \mu \mathrm{m}$ ) supported around its perimeter by a rigid silicon substrate of $200 \mu \mathrm{m}$ thickness. Therefore, much care needs to be taken throughout the sample fabrication processing to finalize the device without breaking the membrane window. The very first step of sample preparation is to clean the substrate. The degree and type of substrate cleaning needed vary with the desired level of substrate cleanliness, and prior processing of the substrate. If the deposited metal is to be used to make electrical contact to the underlying substrate, ensuring that the substrate is clean before processing is crucial. Wafer preparation involves cleaning with acetone (sonication bath for hard substrates which must be avoided for our substrates with membrane window), dip in iso-propanol and blow dry with $N_{2}$ to get rid of dust, debris, contamination or smudges on it. Dehydration bake at $150^{\circ} \mathrm{C}$ can also be performed to get rid of moisture on the substrate.

Resist Selection and Coating: In the next step the substrate is coated uni- 


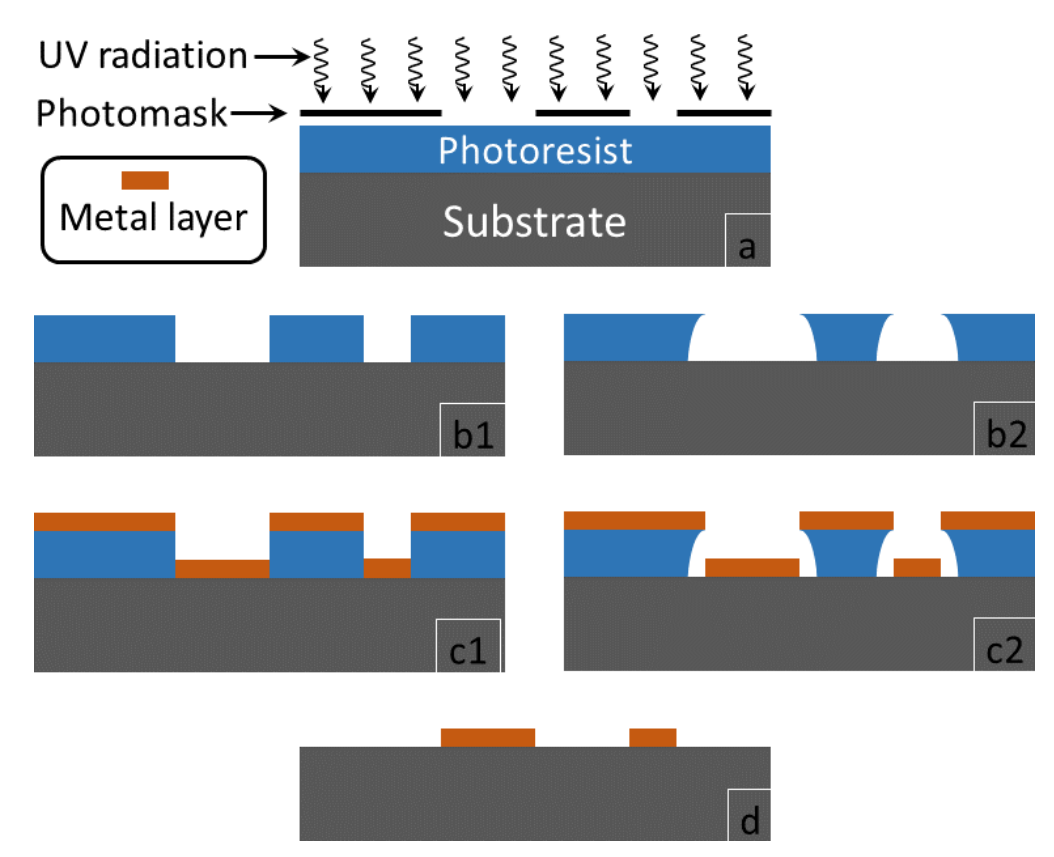

Figure 4.1: Different processes during sample preparation are shown. (a) Photoresist coated substrate is exposed to electromagnetic radiation through a mask. (b1 and B2) The binary pattern after development of resist. (c1 and c2) The sample after metal depostion. (d) Final structures on the substrate after lift-off process.

formly with a radiation sensitive layer, the so called resist. The dyed photoresist versions are recommended to minimize notching and to maintain the linewidth control when processing on highly reflective substrates. For our device fabrication, we used a positive photoresist "Microposit Shipley - 1805", engineered to satisfy the microelectronics industry's requirements for advanced IC devices fabrication. The last two digits of the resist number indicate the optimum thickness for that resist concentration e.g. S1805 is optimized for a resist thickness of $0.5 \mu \mathrm{m}$.

To coat the resist, the substrate is placed into the spinner on a suitable chuck and a viscous liquid solution of photoresist (S1805) is dispensed onto the wafer to cover the substrate. The wafer is spun rapidly typically at $4500 \mathrm{rpm}$ for 30 seconds which produces a resist layer of thickness about $0.5 \mu \mathrm{m}$. The final thickness is also determined by the evaporation rate of liquid solvents from the resist. For very small and dense features, lower resist thicknesses ( e.g. $0.5 \mu \mathrm{m}$ ) are recommended to avoid collapse effects at high aspect ratios - typical aspect ratio is 4:1. This spin coating process produces a uniform layer, usually with uniformity of within 5 to 10 nanometres. This uniformity can be explained by detailed fluid mechanical modeling, which shows that the resist moves much faster at the top of the layer 
than at the bottom where viscous forces bind the resist to the wafer surface. Thus, the top layer of resist is quickly ejected from the wafer's edge while the bottom layer still creeps slowly radially along the wafer. In this way, any 'bump' or 'ridge' of resist is removed, leaving a very flat layer. The photoresist coated wafer is then prebaked to drive off excess photoresist solvent, typically on a hot plate at $90^{\circ} \mathrm{C}$ for 2 - 3 minutes.

Exposure: In this step the exposure of the resist through an optical mask is performed, as shown in Fig. 4.1 (a). A commonly used mask substrate is transparent borosilicate glass or more recently used fused silica because of its lower thermal expansion coefficient and higher transmission at lower wavelengths. Lithographic photomasks are typically covered with a pattern defined with a chrome metal absorbing film. Photomasks are used at wavelengths of $365 \mathrm{~nm}, 248 \mathrm{~nm}$, and $193 \mathrm{~nm}$ and provide a good resolution in the range of $1-2 \mu \mathrm{m}$. In our case of UV-photo lithography a mercury ( $\mathrm{Hg}$ ) arc lamp with a wavelength of $365 \mathrm{~nm}$ is used as the exposure source. According to the desired pattern, absorbing and transparent regions are present on the mask. Consequently, only some regions of the resist are exposed by the UV radiation. The optimum exposure time depends on the resist type, feature size and shape. For our devices and for the resist Shipley-1805, we have used an exposure time of 45 sec.

Development and Post Processing: The next step is the development process, see Fig. 4.2 (b). The exposed resist is removed by a suitable developer solution and thus producing a resist pattern that is a replication of the mask pattern. In the case of the optical resist "Shipley - 1805" the development process is done with a developer named as M351, which is based on buffered $\mathrm{NaOH}$ and used in 1:4 dissolution (with deionised water). For proper development of the exposed resist, we agitate the wafer in the developer for about $45 \mathrm{sec}$ and then rinse it with deionized water for $30 \mathrm{sec}$ to remove the developer from the substrate and to stop further developing process. Thereafter the sample was blown dry with nitrogen and inspected under the optical microscope to confirm a proper lithography process. A post-exposure bake (at 90 to $120^{\circ} \mathrm{C}$ ) was also used sometimes to remove side wall roughness. Note that the developer etches away exposed part of the photoresist much faster than the unexposed part, but the unexposed areas do also become thinner. If you develop for too long, you can etch the unexposed areas too much and render the photoresist mask layer useless.

Optical Lithography is a fast method to replicate big structures many times. The fundamental limit of optical lithography is not determined by the optical system 
only but rather in an overall contribution from the optics, resist and developing processes. To ensure a good lift-off, the metal on the surface of the wafer must not be connected to the metal that is on top of the resist, see Fig. 4.1 (c) and (d). Because of the need for the gap between the metal areas, conformal metal deposition methods, such as atomic layer deposition (ALD) or sputtering are not well suited to lift-off processes. Therefore, we need to be careful if we want to use these deposition methods to grow our structures. In this case, it is good to have an undercut in the resist layer which was done by giving a chlorobenzene treatment to our samples. After exposure, the sample was dipped in chlorobenzene (CB) for 3 min. The sample was taken out of $\mathrm{CB}$ and blown dry with nitrogen. Now the sample was baked at $90^{\circ} \mathrm{C}$ for $3 \mathrm{~min}$. It makes the surface of the resist harder and thus it dissolves less in the developer solution compare to the bottom part and produces an undercut [Fig. 4.1 (b2)] which makes the lift-off process easier.

\subsubsection{Electron Beam Lithography}

With the ability to pattern arbitrary two dimensional structures down to the nanometer scale [119, 120], Electron beam lithography (EBL) is one of the specialized and widely used techniques in nanofabrication. The basic idea behind electron beam lithography is identical to optical or any other lithographies. The substrate is covered with an electron sensitive thin layer of e-resist (in analogy with photoresist for optical lithography), which is chemically changed under exposure to the electron beam. A beam of highly focused electrons is scanned in a well defined pattern across the surface, thus depositing energy in the desired pattern in the resist film to dramatically modify the solubility of a resist material during a subsequent development step. The exposed structure can be developed in proper chemicals so that the exposed/non-exposed areas can be dissolved in a specific solvent (positive/negative lithography) and the pattern can be transferred to the substrate by etching or deposition methods. In addition the electron beam can be applied for beam induced deposition of material supplied by an injected precursor gas. The main attributes of this technique are the very high resolution and the extraordinary flexibility which can be applied to a broad variety of materials and an almost infinite number of patterns. This technique also has some drawbacks e.g. it is slow, being one or more orders of magnitude slower than optical lithography and it is expensive and complicated electron beam lithography tools can cost many millions of dollars and require frequent service to stay properly maintained.

A typical SEM microscope is composed of 1) a column, which is the most im- 
portant part of the microscope. It contains an electron gun (thermionic or field emission), magnetic lenses, which focus and guide the beam, and a specimen chamber. 2) a vacuum system which keeps the column and the specimen chamber at high vacuum $\left(10^{-4} \mathrm{~Pa}\right)$ and 3$)$ the operation and display system which analyses the signals and supplies the electronic signals that guide the electron optics and the vacuum system. The source of the beam is the electron gun, which is positioned at the top of the column. There are two basic types of electron guns: thermionic and field emission sources. A thermionic source is basically a heated wire from which electrons are given enough thermal energy to overcome the work function of the source, combined with an electric potential to give the newly free electrons direction and velocity. A field emission gun consists of a sharply pointed tungsten tip held at several kilovolts negative potential relative to a nearby electrode, so that there is a very high potential gradient at the surface of the tungsten tip. The result of this is that the electrons can escape from the metal by tunnelling. Thermal field emitters enhance the pure field emission effect by giving some thermal energy to the electrons in the metal, so that the required tunnelling distance is shorter for successful escape from the surface. The electron beam is then focused with several magnetic lenses and finally the beam hits the sample. To convert a standard SEM microscope into a basic electron beam lithography writer, only one modification is absolutely necessary, that is to change the signal feed to the scanning coils (which guide the beam over the sample surface) so that instead of drawing a square (like a CRT) the coils will guide the beam to draw an arbitrary pattern.

\subsubsection{EBL Recipes}

The recipe and procedure of electron beam lithography used for our devices is described below:

Substrate Selection: A substrate for electron beam lithography should be relatively conductive to avoid building up an electric charge on it, which could deflect the electron beam and thus distort the drawn pattern. On the other hand the base for electronic circuits should obviously be insulating otherwise the entire circuit would be short circuited. Apparently a compromise must be made in substrate selection - the substrate should be as close to insulating as possible without distorting the drawn pattern. As we are using semiconducting "Si" substrate, there is almost no charging effect in our case. Other possible substrates used for EBL can be glass plates coated with metal (ITO, chrome on glass - widely used in mask production), in this case the metal layer should be grounded before exposure. The 
substrate should also be cleaned as mentioned for optical lithography before further processing.

Electron Beam Resist: To perform electron beam lithography we need a resist, which changes chemically under the exposure of the electron beam. There is quite a large amount of different resists with different properties, which require different chemicals for development and lift-off. One of the first materials developed for e-beam lithography was polymethyl methacrylate (PMMA). It is the standard positive e-beam resist and remains one of the highest resolution resists available. PMMA is available in several molecular weight forms (50K to $950 \mathrm{~K}$ ), usually dissolved in chlorbenzene and currently also in anisol. Depending upon the thickness of the structure to be deposited, different concentrations of PMMA-50K is used and an additional layer of 950K-2\% PMMA is used in a bilayer system to produce an undercut which enables an easier lift-off process as mentioned earlier. The spin coating recipe is as following:

"Spin a 3\% (for structure thickness $50 \mathrm{~nm}$ or below) or $6 \%$ (for thickness between $50 \mathrm{~nm}$ and $150 \mathrm{~nm}$ ) or $9 \%$ (for thickness between $150 \mathrm{~nm}$ and $300 \mathrm{~nm}$ ) mixture of 50K-PMMA and chlorbenzene over the substrate at $3000 \mathrm{rpm}$ for $5 \mathrm{sec}, 8000 \mathrm{rpm}$ for $30 \mathrm{sec}$. Then place the substrate on a hotplate at $150^{\circ} \mathrm{C}$ for 5 minutes for evaporation of excess solvent. Repeat the same spin-coating process with $900 \mathrm{~K}-2 \%$ for a bilayer system and bake the sample for $10 \mathrm{min."}$

As described earlier for an easy lift-off process, there should be a proper gap between the metal layers on the substrate and on top of the resist. This then, is the purpose of the double layer resist. The low weight PMMA is more sensitive than the top layer with higher weight, so the resist develops with an enhanced undercut of the lower resist. In effect, the upper layer is like a shadow mask and the lower layer is a spacer holding the shadow mask off the wafer surface.

Patterning and Exposure: Electron beam lithography is a maskless technology. The pattern is created directly from a digital representation on computer and the electron beam is scanned in a defined pattern across the substrate. In our system all realizations are based on the CAD layout editor (eDraw) for the structure design and the eLitho control software for supervising the lithography process. The software concept is based on the idea of a virtual counterpart to the real sample on the main window of the program. This virtual sample is used to configure the lithography processes to control its operation and to document the sessions. The electron beam lithography uses a finely focussed electron beam scanned over the eresist coated substrate. Good focusing is necessary to minimize the e-beam spot size 
to get better resolution by adjusting stigmation and alignment of aperture. The desired pattern is written as a series of interconnected dots with user adjustable spacings. The e-beam breaks the polymer chains in the resist, making it soluble into a suitable solvent. Most of the time the following parameters are used while exposing our samples during EBL:

1. Accelerating voltage $-25 \mathrm{kV}$

2. Aperture - $20 \mu \mathrm{m}$

3. Working distance $-7 \mathrm{~mm}$

4. Measured beam current - $135 \mathrm{pA}$

The Exposure dose depends upon the pattern size and substrate too. As there are no backscattered electrons from the membrane area, a higher dose is required compared to the hard substrate area. Typically a dose of $600 \mu \mathrm{C} / \mathrm{cm}^{2}$ is used for the membrane area and a dose of $400 \mu \mathrm{C} / \mathrm{cm}^{2}$ outside the membrane. When exposed to more than 10 times the optimal positive dose, PMMA will crosslink, forming a negative resist which is hard to remove afterwards.

Development: For the e-beam resist the exposed parts can be subsequently removed by developing in a suitable solvent. MIBK (methyl-isobuthyl-ketone) alone is a strong developer and removes some of the unexposed resist also, that is the reason a mixture of 1 part MIBK to 3 parts isopropanol is used to develop our structures in a controlled way. The sample is dipped in a 1:3 solution of MIBK (methyl-isobutyl-ketone) and IPA (2-propanol) for $15 \mathrm{sec}$, followed by rinsing in pure IPA for $30 \mathrm{sec}$ to stop the developing process and to avoid removal of ebeam resist from unwanted areas. Then the sample is blown dry with nitrogen and inspected under optical microscope.

Deposition and Lift-off: After the removal of the exposed resist the desired thickness of the metal is deposited on the substrate. Some metals e.g. gold, does not stick properly to the Si substrate, that is why an intermediate adhesion layer e.g. $\mathrm{Ti}, \mathrm{Cr}$ or $\mathrm{Pt}$ is required for better sticking. On the areas exposed to the electron beam the deposited metal sticks to the substrate, while on the unexposed areas the metal sticks to the resist surface. After metal deposition the substrate is soaked in acetone for a couple of hours to dissolve the remaining or unexposed resist (mild ultrasonic agitation is advised for hard substrates to remove the metal around small features) and rinse it with isopropanol. The metal sticking to the resist looses footing and so only the metal sticking to the substrate in desired patterns remains, see Fig. 4.1(d). 


\subsubsection{Metal Deposition Techniques}

Deposition techniques can well be regarded as the major key to produce high quality thin film devices for fundamental research and technology as well. In many instances the characteristics of a thin film can be quite different from the bulk material properties, since thin films have a large surface area to volume ratio. The devices prepared for this research work are grown by physical vapour deposition (PVD) methods which are based on the evaporation or ejection of the material from the source. The advantage of physical methods lay in dry processing, high purity and cleanliness, compatibility with semiconductor integrated circuit processing and epitaxial film growth. However, there are certain disadvantages such as slow deposition rates, difficult stoichiometry control, high temperature post deposition annealing often required for crystallization and high capital expenditure. The three deposition techniques used are sputtering, thermal evaporation and electron beam evaporation. The evaporation system used to deposit normal metals like $\mathrm{Au}$ and $\mathrm{Cu}$ has a base pressure of $\sim 10^{-6}$ mbar. The magnetic structures made of permalloy $\left(N i_{81} F e_{19}\right)$ were grown by a sputter deposition technique having a base pressure of $\sim 10^{-9}$ mbar and an electron beam evaporation technique is used to deposit heavy metals e.g. Ti and Pt as a adhesion layer whenever it is required. The developed chip is loaded into the evaporation chamber and material deposition is performed on top of the sample. In general the deposition process takes place in the following sequential steps:

1. The solid material to be deposited is physically converted to the vapour phase.

2. The vapour phase is transported from the source to the substrate through a region of reduced pressure.

3. The vapour condenses on the substrate to form a thin film.

Although deceptively simple in principle, the skilled practitioner must be well versed in vacuum physics, material science, mechanical and electrical engineering, as well as in elements of thermodynamics, kinetic theory of gases, surface mobility and condensation phenomena. The conversion from the solid to vapour phase is done through physical dislodgement of surface atoms by addition of heat in the evaporation deposition or by momentum transfer in sputter deposition. Evaporants cover an extraordinary range of varying chemical reactivity and vapour pressures. The variety leads to a large diversity of source components including resistance heated filaments, electron beams, crucibles heated by conduction, radiation or rf-induction; 
arcs, exploding wires and lasers. Additional complications include source-container interactions, requirements for high vacuum, precise substrate motion (to ensure uniformity) and the need for process monitoring and control. Considering these points, we will briefly discuss the above mentioned three methods including the advantages and disadvantages of each method.

Thermal Evaporation: Although the thermal evaporation is one of the oldest technique used for deposition of thin films, it is still widely used in the laboratory and in industry for deposition of metals and metal alloys. A large number of materials can be evaporated easily by this technique. If the evaporation is undertaken in a vacuum system, the evaporation temperature will be considerably reduced and the amount of impurities in the growing layer will be minimized. In order to evaporate materials in vacuum, a vapour source is required that support the evaporant and supplies the heat of vaporization which allows the charge of the evaporant to reach a temperature sufficiently high to produce the desired vapour pressure without reacting chemically with the evaporant. To avoid contamination of the evaporant and hence of the growing film, the support material itself must have a negligible vapour pressure. The rate of deposition is controlled by the amount of current supplied through the crucible.

Sputtering: The electrode and gas-phase phenomena in various kinds of glow discharges represents a rich source of processes used to deposit and etch thin films. Creative exploitation of these phenomena has resulted in the development of many useful processes for thin film deposition (as well as for etching). The most basic and well known of these processes is sputtering, the ejection of surface atoms from the electrode surface by momentum transfer from bombarding ions to surface atoms. Since sputtering produces a vapour of electrode material, it is also (and more frequently) used as a method of thin film deposition similar to thermal evaporation technique. Sputter deposition is nothing more than the accumulation of the evaporative atoms which blasted off the target surface onto a nearby sample substrate. During this process, a solid material electrode and substrate are positioned separately within a vacuum system. A high-energy Argon ion plasma stream is targeted at the material, resulting in the subject material being ejected and deposited onto the substrate, creating a thin film.

This method has various advantages over normal evaporation techniques in which no container contamination will occur. It is also possible to deposit alloy films which retain the composition of the parent target material. As this is not an evaporative process, the temperatures required for sputtering are lower than 
thermal evaporation methods. This makes it one of the most flexible deposition processes and it is particularly useful for depositing materials with a high melting point. A mixture of materials and compounds that may evaporate at different rates can also be sputtered at the same rate. Certain processes will benefit from the improved film adhesion due to the higher impact energy.

Electron Beam Evaporation: One way to exert a high degree of spatial and temporal control over the energy input to any processing step is through the use of directed energy beams which are created with both high energy selectivity and spatial directionality. Ion beams, for example, have long been used to implant dopant species with repeatable doses and specific depth profiles. The major advantages of the beam assisted technologies are based on (i) the high dimensional resolution capability (ii) the ability to perform low temperature process and to minimize the thermal exposure of the substrate. Electron-beam evaporation is a physical vapour deposition method for depositing thin films of metals, oxides and semiconductors in a high vacuum environment. An ultra high purity coating material is placed inside a vacuum chamber, typically as pellets in a crucible. The electron energy is used to heat these pellets, causing the coating material to enter the gas phase. Due to the vacuum environment, the evaporated particles can travel to the substrate without colliding with foreign particles. They then condense on the substrate surface to form a thin film. By this technique, high melting point materials can be deposited at high deposition rates, making it a preferred process for refractory metals and ceramic films.

\subsubsection{Atomic Layer Deposition}

Atomic Layer Deposition (ALD) involves the deposition of materials one monolayer at a time and forms extremely uniform, conformal, pin-hole free coatings on high aspect ratio structures. This is achieved by pulsing a precursor onto a hydroxylated substrate. The precursor reacts with this surface but not with itself resulting in a monolayer of material. The precursor is purged from the chamber and then the surface is again hydroxylated with water vapour or oxygen followed by another purge. These two steps are then repeated until the desired thickness of the material is achieved. We have utilized this technique to deposit an insulating layer of aluminium oxide $\left(\mathrm{Al}_{2} \mathrm{O}_{3}\right)$ on our samples. 


\subsection{Fabrication Steps and Samples for Different Ex- periments}

In this section we will explore the device fabrication process step by step and will present different kinds of samples for different experiments. The fabrication of our devices is a multistep process. Each of these steps involves the writing of a specifically designed pattern using EBL or OPL as described previously followed by metal deposition and lift-off. Each subsequent pattern, made of different materials, being printed on the wafer is aligned to the previously formed patterns and built up to form the final device. We have employed a bottom-up strategy to design and fabricate our hierarchical structure which is described below.

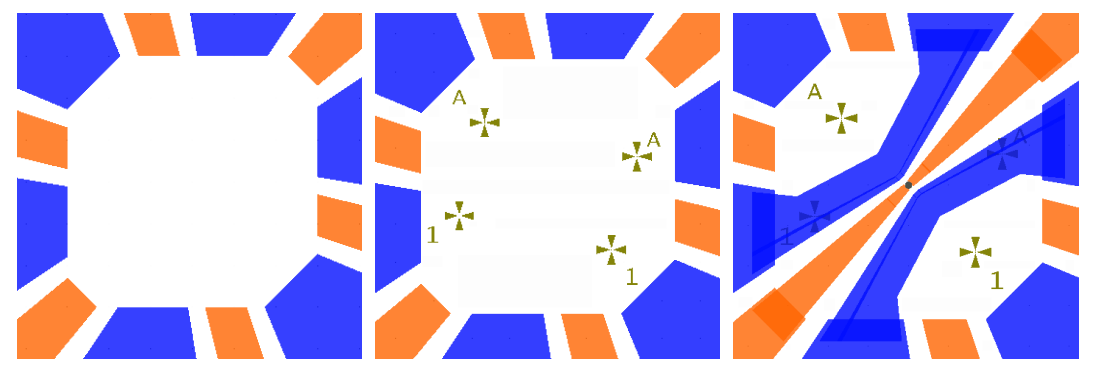

(a)

(b)

(c)

Figure 4.2: Different steps during device fabrication. (a) Outer co-planer wave guide by optical lithograpy. (b) Alignement markers using EBL and (c) different structures of interest in the central part.

Coplanar Waveguide (CPW): In the very first step, the outermost coplanar waveguide is defined by optical lithography (OPL) (Fig. 4.2 (a)). A metal film of $\mathrm{Au}$ with thickness of $120 \mathrm{~nm}$ is deposited by thermal evaporation technique at a base pressure of $4 \times 10^{-6}$ mbar and with a deposition rate of $1-2 \AA / s$. To improve the sticking of gold to the substrate a $10 \mathrm{~nm}$ adhesion layer of $\mathrm{Ti}$ is deposited in situ by electron beam evaporation technique at a rate of about $0.6 \AA / s$. Figure 4.2 (a) shows the central area of our CPW structure having multiple signal lines shown in yellow and ground pads in blue. The small structures of our interest will be defined in the empty space at the center of the structure.

Alignment Markers: The next fabrication step is to prepare some reference structures. These structures are patterned by EBL and consist of four crosses fab- 
ricated as the corner of a rectangle in the central empty space, as shown in Fig. 4.2 (b). These markers are very important for alignment in subsequent fabrication steps to get very well aligned structures in the center part of the device and to recognize each device individually. For these markers, we deposited a Au layer with a thickness of $80 \mathrm{~nm}$ and a Ti sticking layer of $10 \mathrm{~nm}$ under the conditions similar to that for the CPW mentioned in the previous step. As per the requirement for the design of our structures, the positions of these alignment markers can be changed as we will see for the different kinds of samples mentioned below.

Magnetic Structures: The magnetic structures made of Permalloy are patterned by EBL and grown by the sputter deposition method in a base pressure of $1 \times 10^{-9}$ mbar and at a rate of about $1 \AA / \mathrm{s}$. A protection layer of Al-4 nm is deposited at a rate of about $0.25 \AA / s$ to avoid surface oxidation of Permalloy.

Samples for Different Experiments: The additional steps required to prepare different types of samples, will be mentioned.

\section{Anisotropic Magnetoresistance -}

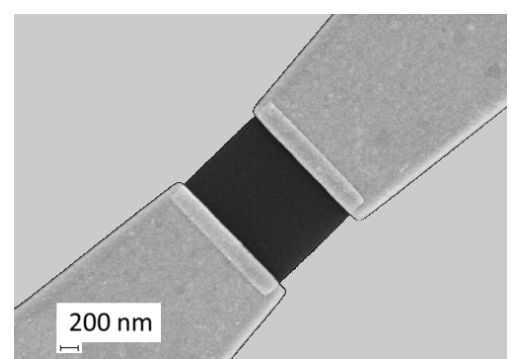

(a) Square

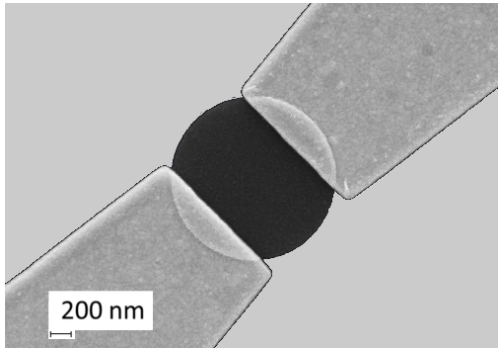

(b) Disk

Figure 4.3: Samples for the AMR experiments to determine the vortex state electrically.

The magnetic squares of edge length $1.6 \mu \mathrm{m}$ and thickness of $50 \mathrm{~nm}$ are prepared in the center of the empty region of outer CPW. These square elements are connected to the outer CPW via two gold electrodes at opposite edges of the element to flow an electric current in the plane of the sample, i.e. the electrons traverse the Py element laterally when the two electrodes lie on unequal electrical potentials. As we have an insulating layer of $\mathrm{Al}_{2} \mathrm{O}_{3}$ on top of the Py layer, it is necessary to remove the insulating layer before the deposition of 
the metal to make a good electrical contact. Therefore prior to the deposition of the metal contact, the Py surface is cleaned in situ by Ar-ion etching for four minutes. This removes the insulating oxide layer at the contact area and ensures a transparent electrical contact. We deposit a thick layer (typically $150 \mathrm{~nm}$ ) of $\mathrm{Au}$ with an adhesion layer of $\mathrm{Pt}(5 \mathrm{~nm}$ ) to overcome the height step of the CPW $(120 \mathrm{~nm})$. In this step the lift-off process has to be done more carefully as the structures are small and the gaps are narrow. Typically the sample is left in heated acetone for several hours.

\section{Spin-Transfer-Torque -}

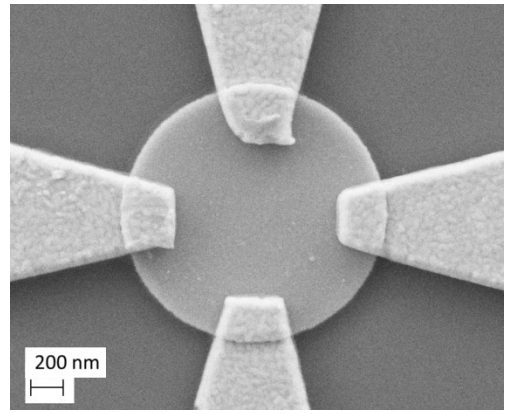

(a) Top view

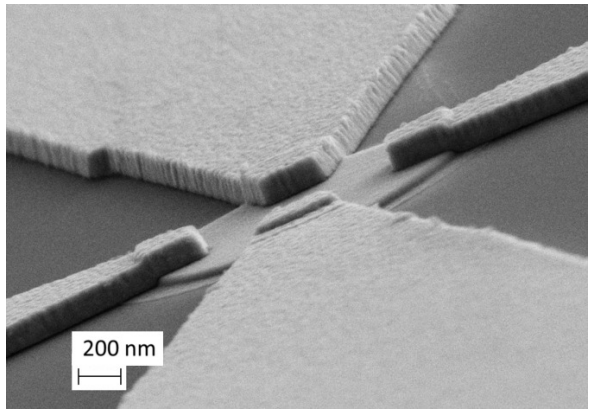

(b) Side view

Figure 4.4: Sample to study vortex core switching assisted by spin-transfer-torque effect. Four electrical contacts are attached to a magnetic disk to flow electrical currents through the sample to generate a rotating current.

Figure 4.4 shows the sample prepared for a detailed study of vortex dynamics in a disk sample of diameter $1.6 \mu \mathrm{m}$, excited with spin transfer torque effect generated by rotating rf currents (similar to the rotating magnetic field described in Chapter.2). The magnetic disk at the center is connected with four $\mathrm{Au}$ contacts located symmetrically at the edge of the disk as shown in Fig. 4.4. The contacts having an area of about $250 \mathrm{~nm} \times 300 \mathrm{~nm}$, were patterned by EBL and the metal deposition is done in the same manner as described above for AMR samples. Some of these samples are also used for AMR measurements.

\section{Magnetic Disk on Top or Below the Crossed Strip Lines -}

Figure 4.5 show the sample design where we have a magnetic disk on top or below the crossed strip-line. As most of the measurements on these samples are performed in STXM, it is required to have a better transmission of X-rays through the whole stack at the area under investigation. Therefore, for these 


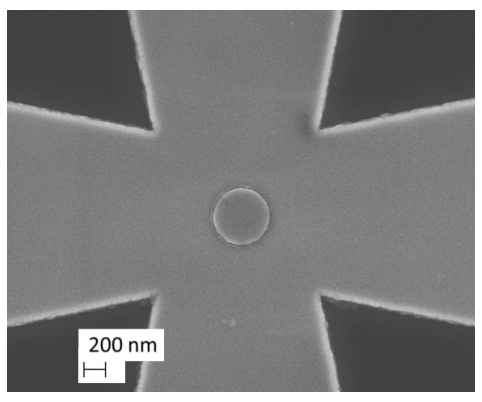

(a) Top view

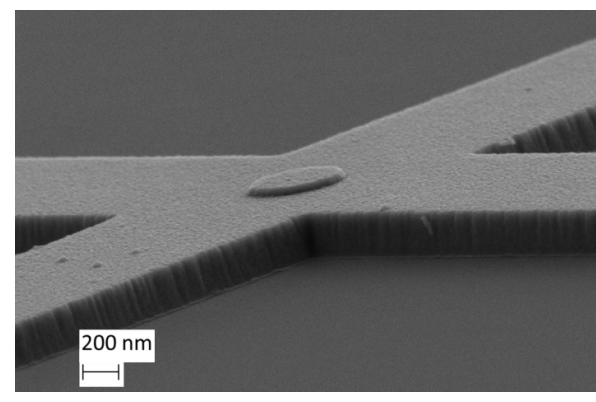

(b) Side view

Figure 4.5: Magnetic disks are deposited on top or below the intersection area of the two striplines. By sending oscillating currents through these striplines an in-plane rotating magnetic field can be obtained in the intersection area.

samples the striplines are made of copper which is good conductor and is more transparent to the X-rays. Disks of diameter $500 \mathrm{~nm}$ and $1.6 \mu \mathrm{m}$ are prepared on top of crossed stripline of width $1.5 \mu \mathrm{m}$ and $2.5 \mu \mathrm{m}$ at the narrowest part, respectively. According to the demand of the experiment the thickness of the disk varied from $10 \mathrm{~nm}$ to $100 \mathrm{~nm}$, covered with a $4 \mathrm{~nm}$ protecting layer of Al. The ALD technique is used to deposit an insulating layer of $\mathrm{Al}_{2} \mathrm{O}_{3}$ ( $5-10 \mathrm{~nm}$ ) between the disk and stripline to avoid a direct contact between the two. Additionally, some samples of this type are prepared with an additional layer of out-of-plane magnetized GdFe over the Permalloy disk. Here GdFe is deposited at the Max-Planck-Institute for Intelligent Systems, Stuttgart. Several experiments are performed with these types of samples, the experimental results of which will be presented briefly in Chapter.6.

\section{Sample for the Spin-Motive-Force Measurements -}

Figure 4.6 shows the sample for SMF measurements. This sample is similar to the samples prepared on a crossed stripline. There are two additional bridge contacts on the magnetic disk to measure the oscillating spin-motive-force signal. These bridge contacts are prepared by EBL using two layer resist where the bottom layer is less sensitive (hard) to the electron beam compare to the top layer (soft). The exposure dose and developing time is chosen in such a manner that only the top layer resist at the desired places is removed after development and the bottom layer stays there. After metal deposition and lift-off process, the bottom resist is removed and we end up with a bridge like structure. Special attention has been paid to avoid shortening of disk and 


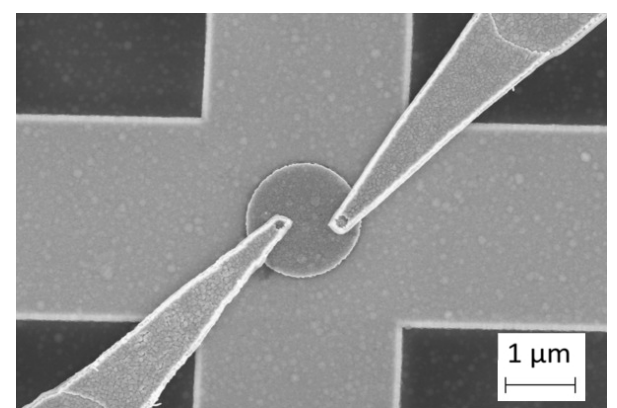

(a) Top view

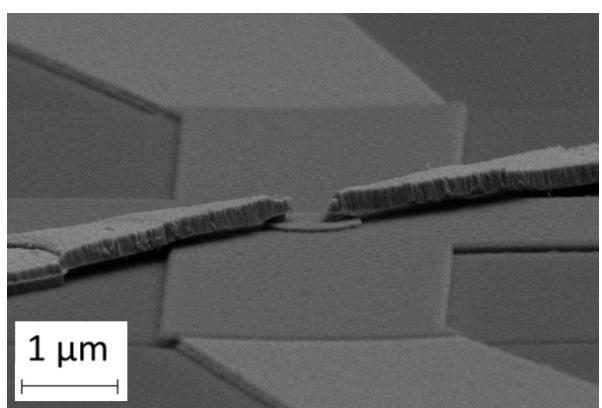

(b) Side view

Figure 4.6: Sample to investigate the SMF signal due to the gyrotropic motion of the vortex core. The magnetic disk at the intersection area of the two striplines is insulated by a $25 \mathrm{~nm}$ thin layer of $\mathrm{Al}_{2} \mathrm{O}_{3}$ deposited by the $\mathrm{ALD}$ method. Two bridge like contacts of contact area diameter of about 100 and $150 \mathrm{~nm}$ are prepared over the disk to measure the SMF signal.

bridge contacts with the striplines, by covering the crossed stripline with 25 nm of insulating $\mathrm{Al}_{2} \mathrm{O}_{3}$ layer by atomic layer deposition technique.

The two bridge contacts are at asymmetric position with respect to the center of the disk. One contact is close to the edges of the disk while the other one is closer to the centre of the disk (but not exactly at the centre). This configuration on one hand ensure an asymmetric magnetic texture between the contacts when the vortex is brought into orbit. On the other hand, by placing the contacts outside the orbit of the gyrating vortex, we avoid pinning induced by lithographic processes. The electric contacts are insulated from the stripline and disk except for the small $(\sim 75-150 \mathrm{~nm}$ diameter $)$ contact areas on the disk. This sample geometry allows us to detect induced voltages in various configuration - linear and circular excitation fields, voltage probes collinear and perpendicular to the linear excitation. Also the vortex core polarity can be controlled by selective switching with co-rotating in-plane magnetic fields.

\section{Radial Spin Waves -}

Figure 4.7 shows the next type of sample used to study radial spin wave modes in thin Permalloy platelets in the vortex ground state. These samples are not measured in STXM and do not require transmission of X-rays or election, therefore, these samples are prepared on hard substrates and the CPW is made completely out of gold metal. These samples consist of Permalloy disks of 


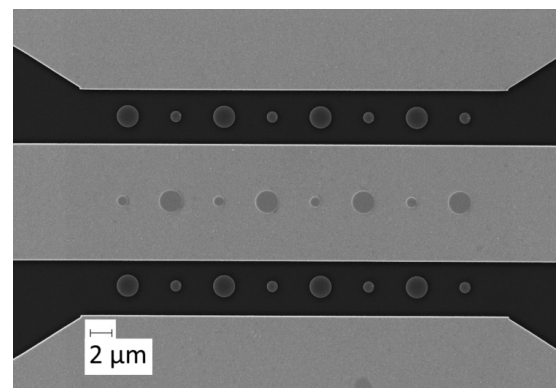

Figure 4.7: Sample to investigate radial spin waves in vortex structures.

varying diameter $(1-3 \mu \mathrm{m})$ and thickness's $(10-50 \mathrm{~nm})$, located on top and in the gap of a gold coplanar waveguide (CPW). The disks were separated by at least twice the diameter of the disk to avoid dipolar coupling. The structures were created directly on a silicon substrate using thermal evaporation and electron beam lithography. The entire sample was covered with a conformal layer of aluminum oxide ( $7 \mathrm{~nm}$ ) by Atomic Layer Deposition (ALD) to prevent oxidation.

\section{Antidot Lattice -}

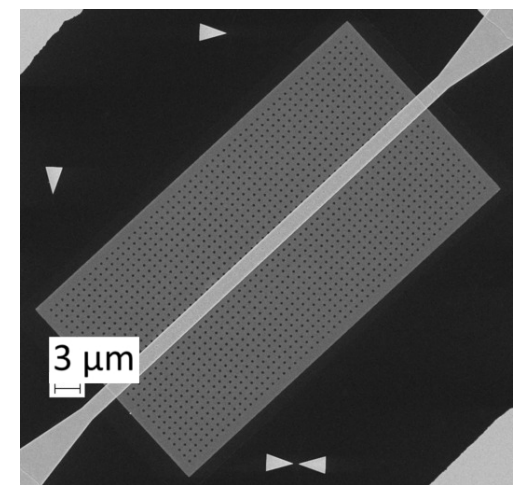

(a)

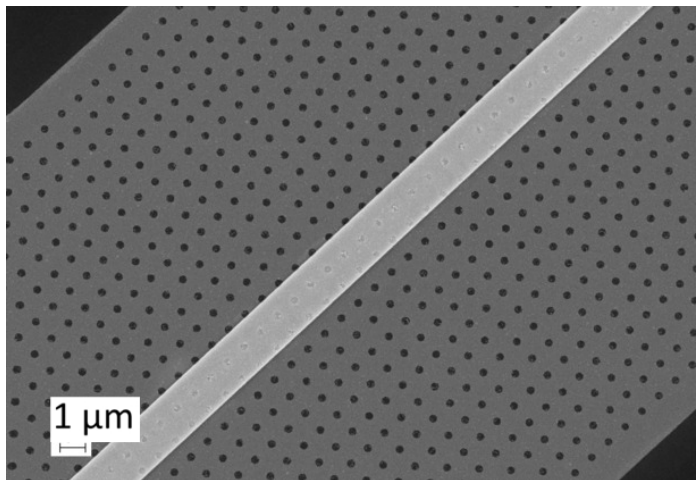

(b)

Figure 4.8: Antidot lattice to study the propagation of spin waves in these structures.

Figure 4.8 shows the sample to investigate the anisotropic spin-wave propagation in a magnetic antidot lattice made of Permally [121]. The antidot lattice is prepared by EBL and lift-off process. The diameter of the antidot is about $450 \mathrm{~nm}$ and the center to center distance between two neighbouring antidots is $900 \mathrm{~nm}$. The lattice is covered with an insulating layer of $\mathrm{Al}_{2} \mathrm{O}_{3}$ deposited by ALD technique. A stripline of width $2 \mu \mathrm{m}$ and made of $\mathrm{Cu}$ is prepared on 


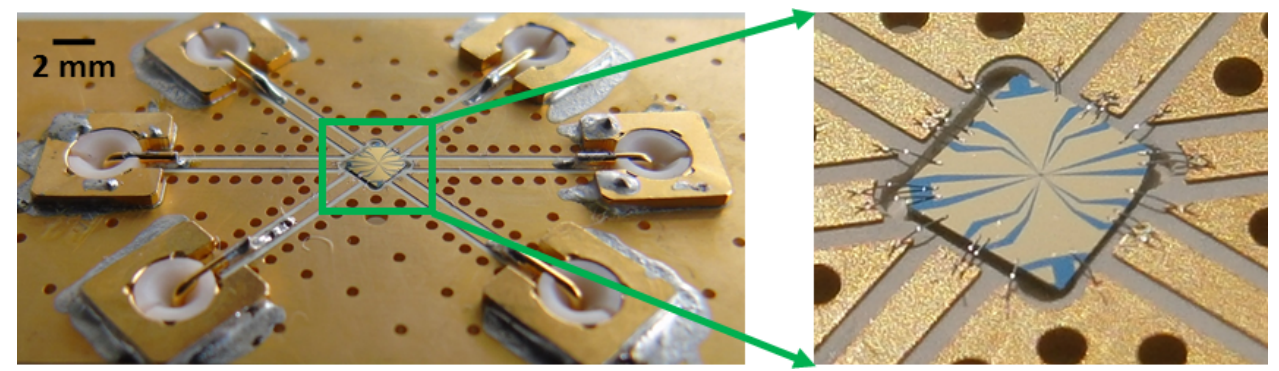

Figure 4.9: The sample mounted and bonded on a holder with multiple rf connectors.

top to generate a magnetic field to excite spin waves in the lattice.

Inspection and Sample Mounting: As we have already seen, scanning electron microscopy (SEM) images are taken to visualize the shape of the prepared samples and to verify the lateral dimensions of these micro-structures. Atomic Force Microscopy (AFM) was also utilized to investigate the roughness and thickness of the deposited metal films. AFM imaging showed the deposited Permalloy to have a random polycrystalline microstructure with the grains of about $10 \mathrm{~nm}$ in size and a surface roughness of less than $1 \mathrm{~nm}$. After the processing is finished, the sample is glued onto a sample holder having several rf-connectors and bonding wires are attached from the holder to the bonding pads (outer CPW) on the sample, see Fig. 4.9. 
CHAPTER 5

\section{Instrumentation and Measurement Techniques}

In this chapter we will be acquainted with the measurement techniques and setup utilized during the research work presented in this thesis. The measurements are partially performed at "MAXYMUS endstation of beamline PGM II 46U, BESSY II, Berlin" and mostly at University Regensburg. The basic circuits used for these measurements and evaluation methods to obtain physical parameters will be presented in the following sections. The circuits may be modified slightly to accommodate special measurement requirements. The control programs employed to improve experimental possibilities and analyses will also be discussed in the course of this chapter.

\subsection{Scanning Transmission X-ray Microscopy}

\subsubsection{Synchrotron and Generation of X-rays}

During the last decade, the dramatic improvements in the performance of the new generation synchrotron radiation sources producing intense tunable monochromatized X-ray beam has opened up new possibilities in fundamental research. Synchrotron radiation is a common light source for a variety of experiments ranging from physics, chemistry, material science to bio-science research. A synchrotron source basically consists of a storage ring with electrons circulating at $\mathrm{GeV}$ energies which corresponds to a speed near to that of light. The electron beam travels in the storage ring and is maintained by a strong magnetic field. The storage ring is not actually circular, but a set of straight sections connected by strong magnets which bend the electron beam.

BESSY II employs a TV-like device to generate a $70 \mathrm{keV}$ electron beam. Before injection in the main storage-ring the beam is accelerated over a microtron and a synchrotron to its final energy of $1.7 \mathrm{GeV}$. The accelerating process takes $50 \mathrm{~ms}$ and can be repeated with a repetition rate of $10 \mathrm{~Hz}$. The total current of about 
$300 \mathrm{~mA}$ in the storage ring can be obtained by successive injection of electrons, accelerated in multiple accelerating cycles. High energy electrons moving with a speed close to the speed of light, pass through a periodically arranged magnet system, the so called "Undulator" which are installed in straight sections of the storage ring. Such an undulator consists of a periodic arrangement of magnets with relatively weak magnetic fields and induce a rotating magnetic field along the electron beam. The electrons travelling through such undulators experience acceleration motion and emit energy in the form of intense elliptically polarized X-rays. These X-rays are channeled along "beamlines" - into laboratories where they are used to analyse structures in extraordinary details. The energy loss of the electron beam is compensated by oscillating radio frequency cavities in the ring. The arrangement of the magnets in the undulator can be changed to generate $\mathrm{X}$ rays of desired energy and polarization, therefore, it is a perfect light source for (magnetic) X-ray microscopy. The electrons in the storage ring are not distributed uniformly but bunched in evenly spaced buckets and the circumference of the ring has to be exactly a multiple of the bucket spacing. The distance between these buckets at BESSY II is $60 \mathrm{~cm}$ which corresponds to the ring frequency:

$$
f_{\text {ring }}=\frac{c}{60 \mathrm{~cm}} \simeq 499.654 \mathrm{MHz}
$$

which yields 400 buckets. Depending on parameters like temperature or the exact steering of the beam orbit in the ring, the exact ring frequency in practice may differ slightly from the value given in the above equation. Therefore, active and continuous synchronization of the time base of dynamic experiments with the ring frequency is required. The width of the electron bunch inside those buckets is of the order of $25-100 \mathrm{ps}$, depending on the synchrotron as well as the beam current. At BESSY II, a typical bunch width in normal operation is around 70 ps. The default operation mode of the synchrotron relevant to this work is called multibunch mode where most buckets are filled with an average amount of current.

\subsubsection{Contrast Mechanism: The XMCD Effect}

Several techniques exist to measure the magnetic properties of materials. Most of them are sensitive to the total magnetization of the measured system and can not discern between the contributions of different atoms in an alloy or multilayer. Moreover, the small quantity of material present in many technologically interesting samples, like magnetic nanostructures, necessitates a very sensitive measuring method. The contrast mechanism we used to image the magnetization dynamics 


\subsection{Scanning Transmission X-ray Microscopy}

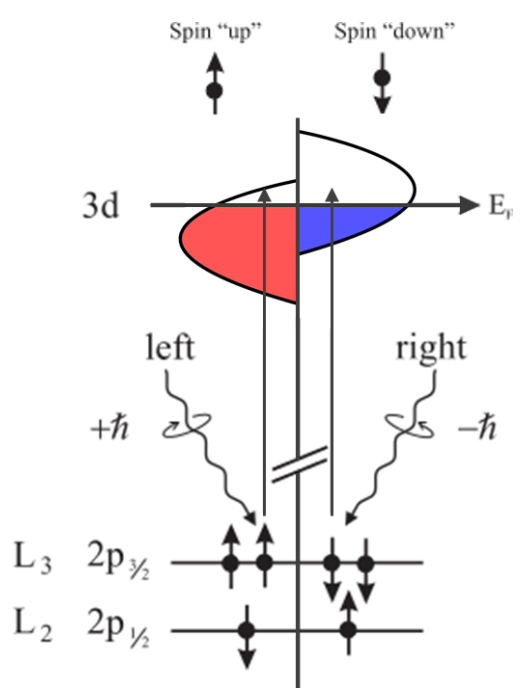

(a)

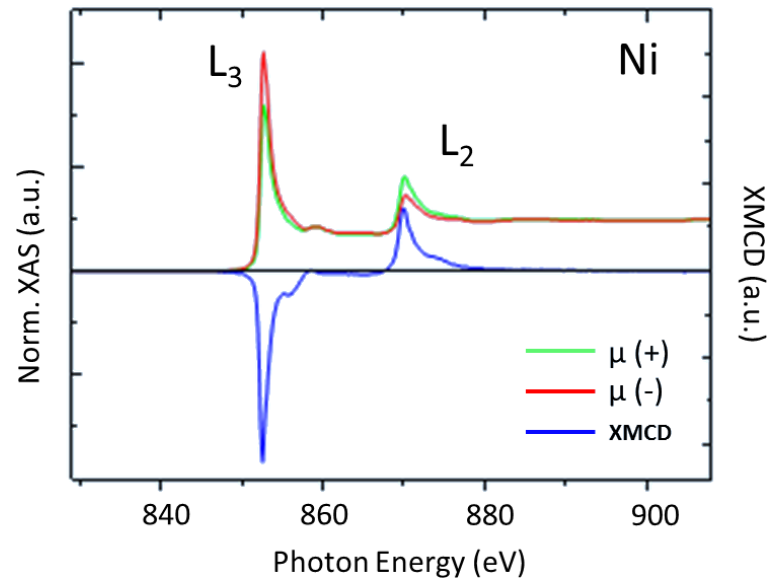

(b)

Figure 5.1: (a) Schematic description of the XMCD effect at the $L_{2,3}$ absorption edges for $3 d$ transitions metals. Absorption of left (right) circularly polarized x-rays leads to excitation of spin up (down) electrons from $2 p$ states to $3 d$ states. (b) Ni absorption spectra for left (red) and right (green) circularly polarized light. The XMCD spectrum is shown in blue (Reference [122]).

is the X-ray Magnetic Circular Dichroism (XMCD) effect, the first experimental proof of which is given by Schütz et al. in 1987 [95]. This is a quantum mechanical effect that causes the absorption coefficient for circularly polarized monochromatic X-rays of a magnetized medium to depend on the direction of the magnetization in a ferromagnetic sample, see Fig. 5.1. The absorption coefficient $(\mu)$ can be described by Fermi's golden rule within first order perturbation theory:

$$
\mu(\hbar \omega) \propto \sum_{f}\left|\left\langle\psi_{f}|\mathbf{p} \cdot \mathbf{A}| \psi_{i}\right\rangle\right|^{2} \delta\left(E_{f}-E_{i}-\hbar \omega\right)
$$

Where $\psi_{f}$ and $\psi_{i}$ are the final and initial states with energies $E_{f}$ and $E_{i}$, respectively. The quantity $\mathbf{p}$ is the momentum operator and $\mathbf{A}$ is the vector potential of the incident electromagnetic field.

In X-ray absorption, the atom absorbs a photon, giving rise to the transition of a core electron to an empty state above the Fermi level obeying the selection rule $\left(\Delta m_{l}= \pm 1\right)$. The absorption cross-sections are large, especially in the soft $\mathrm{X}$-ray range $(500-2000 \mathrm{eV})$. The absorption edges have energies which are characteristic for each element and, due to the dipole selection rules, final states with different symmetries can be probed by choosing the initial state. Because the effect 
only occurs near an absorption edge, it is element specific and thus can also be used to image the magnetization of different materials in a stack geometry. Since the absorption depends only on the magnetization component along the photon propagation direction, the XMCD effect is sensitive to one component of the magnetization, which can be chosen by varying the angle of incidence of the incoming photon beam.

The properties of $3 \mathrm{~d}$ transition metal elements $\mathrm{Fe}$, Co and $\mathrm{Ni}$ are largely determined by the $3 \mathrm{~d}$ valence electrons. Since X-ray absorption spectra are governed by dipole selection rules, the d-shell properties are best probed by L-edge absorption studies $(2 \mathrm{p} \rightarrow 3 \mathrm{~d}$ transition). For these edges the transitions take place directly to the empty $3 \mathrm{~d}$ states, which are strongly polarized. The XMCD effect measured at the $L_{2,3}$ edges of these elements was found to be as large as $20 \%$ of the total absorption [95, 122]. In the case of Fe, the dichroic contrast is as large as $40 \%$. A real explosion of the use of XMCD has followed the development of sum rules by Thole et. al. [123]. These sum rules, applied to the total absorption and XMCD spectra, allow to obtain direct values for the orbital and spin moment of the probed atom.

\subsubsection{STXM Setup}

The observations of magnetic structures are accomplished by magnetic transmission soft X-ray microscopy with spatial resolution down to 25 nanometers, thanks in part to the high quality X-ray optics provided by BESSY researchers. The scanning transmission X-ray microscope (STXM) principle is a simple one, see Figs. 5.2 and 5.3. The elliptically polarized monochromatic photon beam provided by the undulator beam line is focused to a small spot of less than $25 \mathrm{~nm}$ on the sample, using a Fresnel zone plate lens in combination with an OSA (Order Sorting Aperture). An image is constructed by scanning the sample in the focal plane of the zone plate in the horizontal and vertical direction with a high accuracy translation stage while the intensity of the transmitted photon flux is measured as a function of the sample position. To enable the separate detection of transmitted photons belonging to different flashes, we use a fast Avalanche Photo Diode (APD). This type of detector responds very fast to individual photons, and it gives well-separated output pulses corresponding to each of the photon flashes. The lateral resolution of this technique is limited by the size of the focus spot of the zone plate and is about $20-30 \mathrm{~nm}$. This resolution is well suited to image the magnetization state of nanostructures. The XMCD effect is sensitive to the projection of the magnetization on the photon 


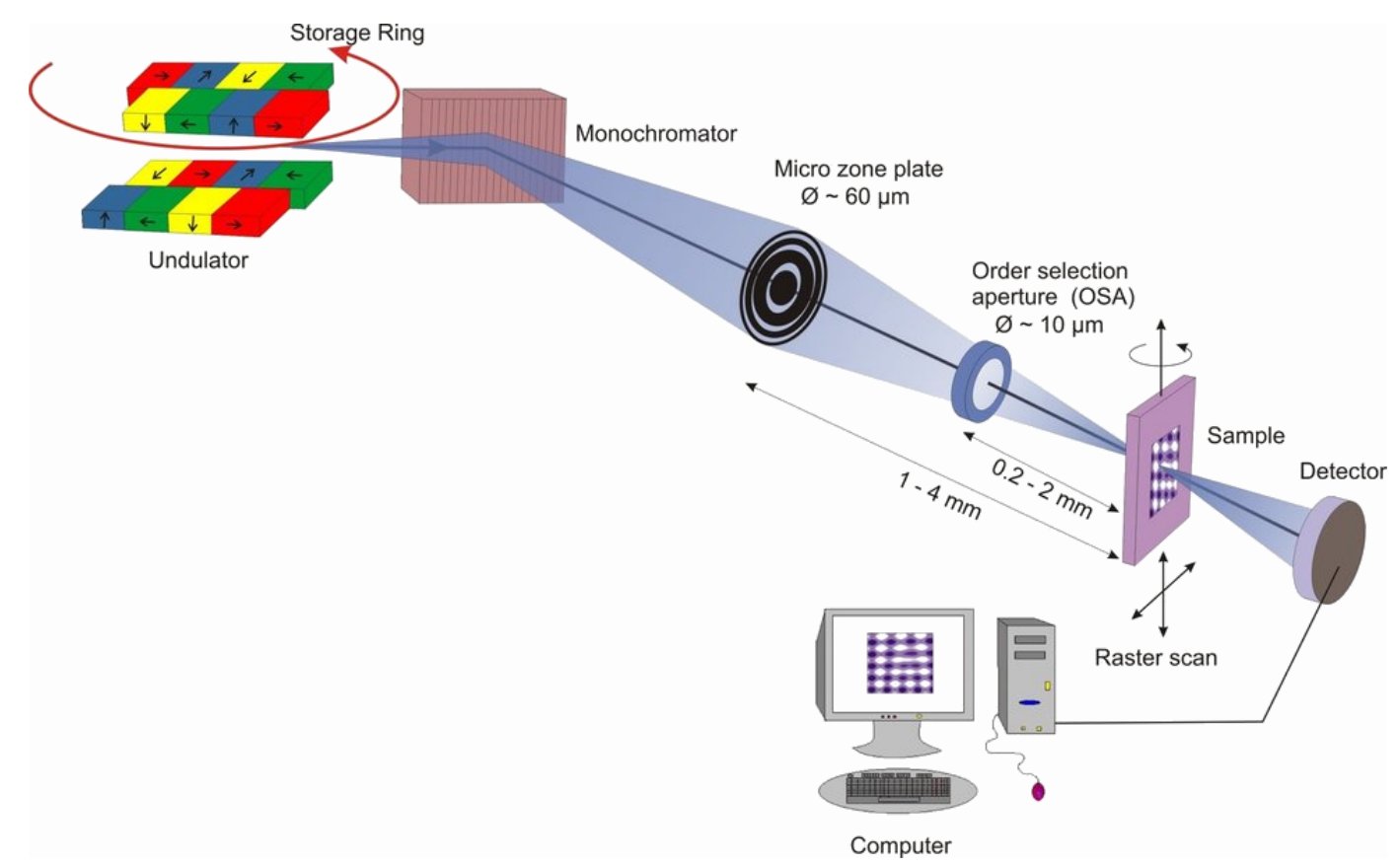

Figure 5.2: Sketch of scanning transmission X-ray microscopy technique using synchrotron radiation.

propagation direction. The in-plane magnetization state or circulation direction in our vortex structures can be imaged by tilting the plane of the sample at an angle of 30 degree with respect to x-ray propagation direction. The resolution of STXM even allows to image the out-of-plane magnetized vortex core itself. Here we let the photon beam hit the sample perpendicularly, so that we only image the z-component of the magnetization.

STXM not only offers an increase in lateral resolution by using soft X-rays, but also the inherent pulsed time structure of synchrotron radiation sources makes time dependent magnetic studies feasible. At BESSY-II, electron bunches with a pulse length of each 70 ps and separated in time by 2 ns are circulating for several hours in the storage ring. To study magneto-dynamics we have used a stroboscopic pump-and-probe experiment triggered by the orbital clock of the storage ring. Note that, in this timing structure, only very limited phases can be probed by the x-ray pulses, thus the delay between the pump and the probe is often varied to study more phases of the sample dynamics. Typically, the magnetization dynamics is excited by a pump which is synchronized to the probe, the X-ray pulses. The pump can be either pulses or continuous signals. The probe is the X-ray flash of the synchrotron which is delayed with respect to the pump pulse to study the temporal evolution of the magnetization. Thus, the development of time resolved X-ray 


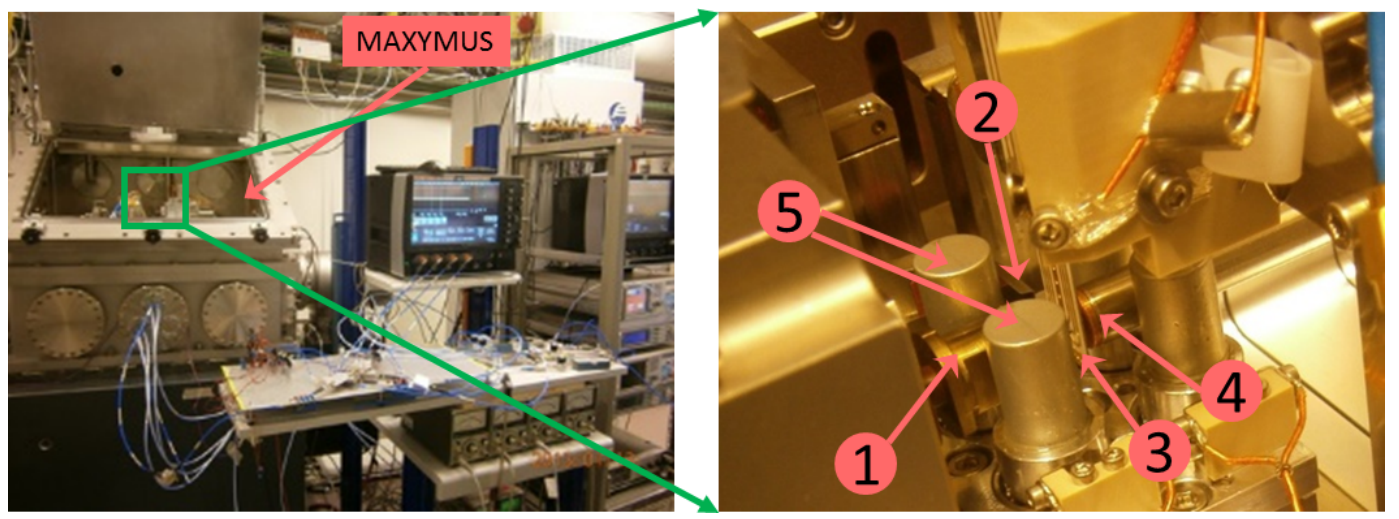

Figure 5.3: STXM setup: The left image shows an outside view of the STXM chamber at MAXYMUS. The right image shows an inside view with a sample mounted between magnetic system. (1) Zone plate, (2) OSA, (3) Sample, (4) Detector and (5) Magnet system.

imaging provides opportunities to study the vortex dynamics with high spatial and temporal resolution.

\subsection{Electrical Measurement Setup}

\subsubsection{AMR Setup}

The set-up constructed for the electrical measurements of vortex dynamics is used to perform experiments including AMR and SMF measurements. A sketch of the electrical circuit is shown in Fig. 5.4 and some minor modifications are done whenever demanded. To inject high frequency signals into the sample, a broadband microwave signal generator "Aeroflex IFR-3416" is used which covers a frequency range from $250 \mathrm{KHz}$ to $6 \mathrm{GHz}$. This frequency range is more than sufficient for our experiments. In order to generate an in-plane rotating current to switch the vortex core polarity selectively in the disk sample with four electrical contacts, the main signal from the signal generator is split into four branches as depicted in Fig. 5.4(a). Initially the signal is split into two branches via a 90 degree splitter [ZMSCQ-2$180+(120-180 \mathrm{MHz})$ or ZX10Q-2-3+ $(220-470 \mathrm{MHz})]$. The split signal passes through a transfer switch "MTS-18-12B+" which operates at $12 \mathrm{~V}$ dc voltage supply and allows the exchange of the output signal. These two output signals are then split again via a 180 degree splitter "ZFSCJ-2-4+" which works in the frequency range from $50 \mathrm{MHz}$ to $1 \mathrm{GHz}$. Consequently, we obtained four signals (A1, A2, B1 


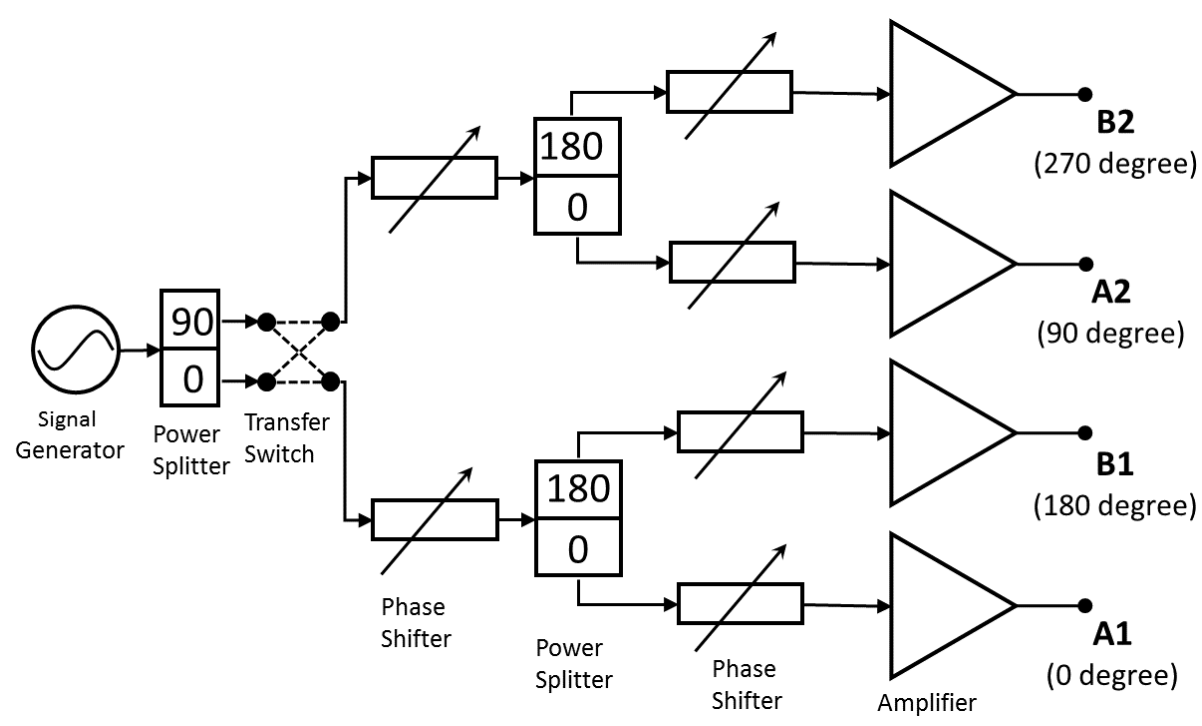

(a) Splitting of the rf signal

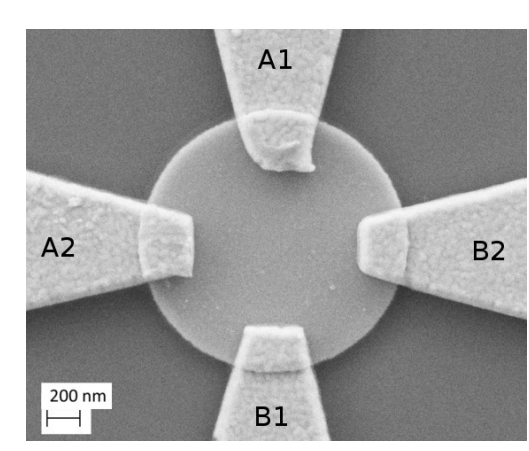

(b) Rotating excitation

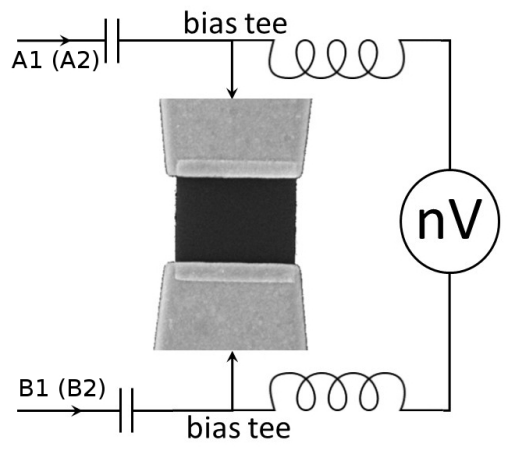

(c) Linear excitation

Figure 5.4: Sketch of the excitation schemes.

and B2) having the same amplitude and at fixed phase (0, 90, 180 and 270 degree). Some additional rf components are also used to obtain experimental requirements. Care has been taken to use rf cables of the same kind and of equal length in each branch to maintain the symmetry to obtain output signals at the required phase. The four output signals are inspected in an oscilloscope "Tektronix TDS 540B" and mechanical phase shifters are used to adjust small phase differences if needed. Some rf attenuators are also used to reduce the problem of impedance mismatch while connecting different rf components. Broadband (5 MHz to $1.5 \mathrm{GHz}$ ) and low noise (typically $1.2 \mathrm{~dB}$ ) amplifiers "KU LNA BB 0515 A-SMA" are used to balance the power loss due to the attenuators used in the electronic circuit and to increase the power, sufficient enough to excite the vortex core dynamics and to switch the vortex 


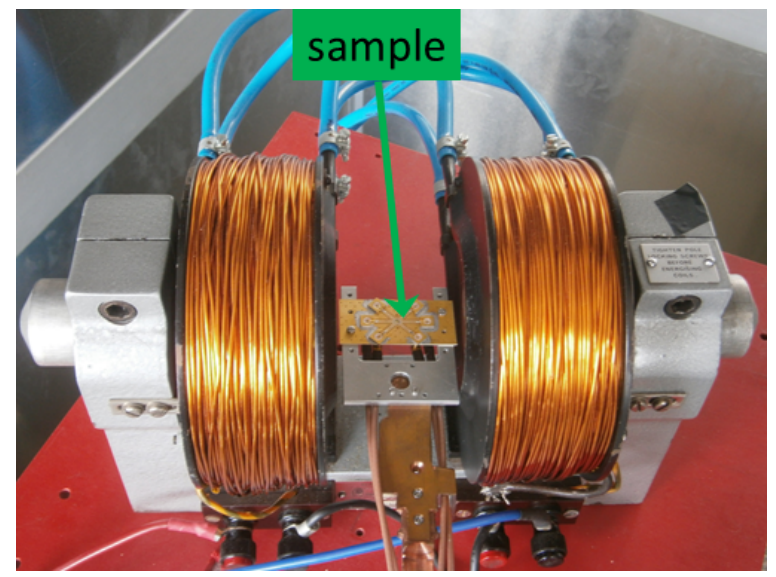

Figure 5.5: The sample is located between the pole pieces of an electromagnet.

core.

The disk samples with four electrical contacts can be excited by rotating currents by injecting these four signals in four contacts in a schematic manner as shown in Fig. 5.4(b). To generate a rotating current at the sample, the rf signal in any two consecutive contacts should have the same amplitude and a phase difference of 90 degree. By changing the phase of these signals, the rotation sense of the current can easily be changed from counterclockwise (CCW) to clockwise $(\mathrm{CW})$ or vice-versa and in our circuit this is possible by interchanging the signals through a transfer switch.

One pair of these outputs (either A1 - B1 or A2 - B2) is used to excite the magnetic structure with linear current, as shown in Fig. 5.4(c). Figure 5.4(c) shows the sketch of our experimental setup for the homodyne detection of the AMR signals generated due to the gyrotropic motion of the vortex core. The sample is mounted between the pole pieces of a water cooled electromagnet 5.5, used to apply an external static magnetic field in the plane of the sample. This electromagnet can produce a magnetic field of more than $100 \mathrm{mT}$, sufficient for our experimental requirement to saturate the magnetic sample. Measurements are controlled by using a GPIB interface to a computer running LabView which allows running sequences of arbitrary measurements. The transmission rf lines which guide the rf signal from the signal generator to the sample with minimal losses consist of semi-rigid coaxial cables, CPW on the sample holder, the coplanar waveguide fabricated on chip and the connections between them. Special care needs to be given to minimize the reflection at these connections. A coplanar waveguide (CPW) is designed for the sample holder and on the sample itself to match $50 \mathrm{Ohm}$ characteristic impedance 


\subsection{Electrical Measurement Setup}

of the coax line to minimize the power loss due to reflections.

To separate the microwave current and DC lines without any disturbance, a biastee "ZFBT-282-1.5A+" is used as shown in Fig. 5.4(c). Bias tees are passive coaxial components primarily used in applications where you need to inject DC currents or voltages into RF circuits [124]. The bias tee is a duplexer. Conceptually, the bias tee can be viewed as an ideal capacitor that allows AC through but blocks the DC bias and an ideal inductor that blocks AC but allows DC. Thus, the low frequency port is used to set the bias; the high frequency port passes the radio frequency signals but blocks the biasing levels; the combined port connects to the device, which sees both the bias and RF. It is called a tee because the three ports are often arranged in the shape of a "T". The microwave current is injected into the magnetic sample via the high frequency port of the bias-tee and the rectified homodyne (DC) voltage signal is measured in a nanovoltmeter via the dc port of the bias tee.

\subsubsection{SMF Setup}

Spin motive force measurements are performed for a disk sample of diameter $1.6 \mu \mathrm{m}$. The vortex dynamics in these samples were excited with in-plane rf fields generated by flowing rf currents in the striplines below the magnetic sample. The excitation scheme is the same as described above for AMR measurements, see Figs. 5.4(a) and (b). The four signals (A1, A2, B1 and B2) are injected into the crossed striplines [see Fig. 5.6 (a)] to generate linear or rotating in-plane oscillating magnetic fields at the intersection area of the striplines. The potential drop due to SMF is measured via two bridge contacts ( $\mathrm{C} 1$ and $\mathrm{C} 2$ ) fabricated over the disk as sketched in Fig. 5.6. The rf signal picked up by two contacts is subtracted via a combiner "ZFSCJ-2$4+$ ". A 3-dB attenuator is used to reduce the problem of impedance mismatch. As the SMF generated voltage signal is very small, as mentioned in Chapter-3, the subtracted signal is passed through a chain of amplifiers to increase its level sufficiently to be measured in an oscilloscope "Tektronix TDS 540B". The chain of amplifiers is made by combining several amplifiers with other rf components, e.g., attenuators and limiters, to take into account the limitations of the used amplifiers. Additional rf filters are used to decrease the noise level in the measured signal. Similar to the AMR measurements, these samples are also mounted between the pole pieces of the same electromagnet and measurements are performed with and without external fields. 


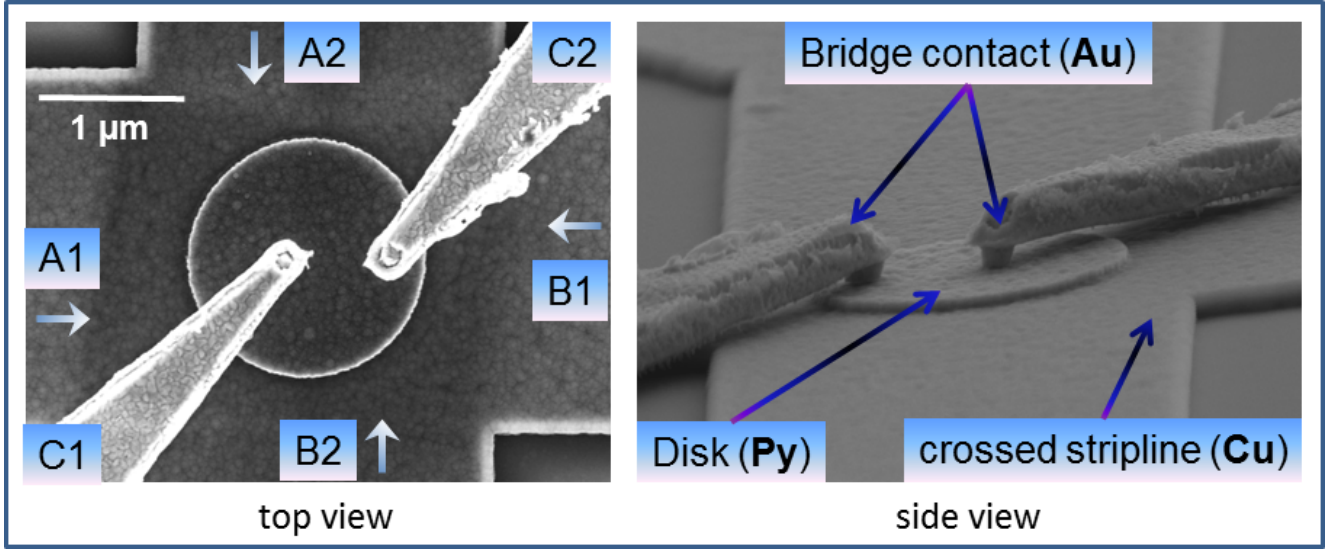

(a)

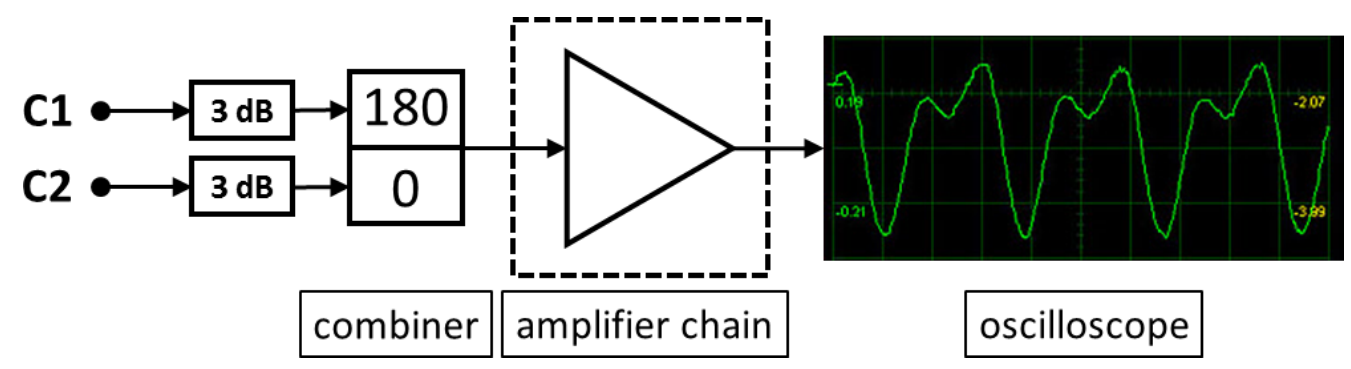

(b)

Figure 5.6: Schematics of the Spin Motive Force measurements setup. 
The theoretical background of magnetic vortex dynamics and techniques to image and detect it by electrical means is illustrated in the previous chapters. The results obtained from the previously mentioned techniques will be presented throughout this chapter. The time dependent spatial distribution of the magnetization was imaged using a scanning transmission X-ray microscope. The results from the stroboscopic technique, utilized to image the gyrotropic motion of the vortex core excited with rf currents or magnetic fields will be presented. It will be followed by a detailed description of the results obtained from measurements to detect the vortex core by electrical means. The results obtained from some collaboration projects will also be presented briefly.

\subsection{STXM Measurements}

\subsubsection{Static Imaging}

In-plane: This section focuses on the characterization of magnetic microstructures, containing a single vortex in remanence, by imaging in a scanning transmission Xray microscope described in Chapter-5. We have imaged both the in-plane and outof-plane magnetization distribution in our samples. In order to visualize the spatial distribution of the static in-plane magnetization $(\vec{M}(\mathbf{r}))$, the sample has to be placed under an oblique angle so that there is a contribution of the in-plane magnetization along the photon propagation direction. Therefore, the sample was placed at an angle of $30^{\circ}$ with respect to the direction of propagation of X-ray photons. In this configuration, the observed magnetic contrast is dominated by the contribution from the in-plane magnetization and thus the orientation of in-plane magnetization or circulation of the vortex structure can be determined. The elemental sensitivity of X-rays allows us to isolate signals of the magnetic layer from other layers by tuning the X-ray energy to the absorption edge of the element to be probed. As the material (Permalloy) under investigation has a high concentration (81\%) of nickel, 
the data were recorded at the $L_{3}$ absorption edge $(852.7 \mathrm{eV})$ of $\mathrm{Ni}$, at which the XMCD effect gives a strong magnetic contrast (Fig. 5.1).

The transmission spectrum (as shown in Fig. 6.1) through the Permalloy sample was recorded to adjust the undulator to provide the energy of $\mathrm{Ni} L_{3}$-absorption edge and the energy was set near to the minimum of this spectrum for further

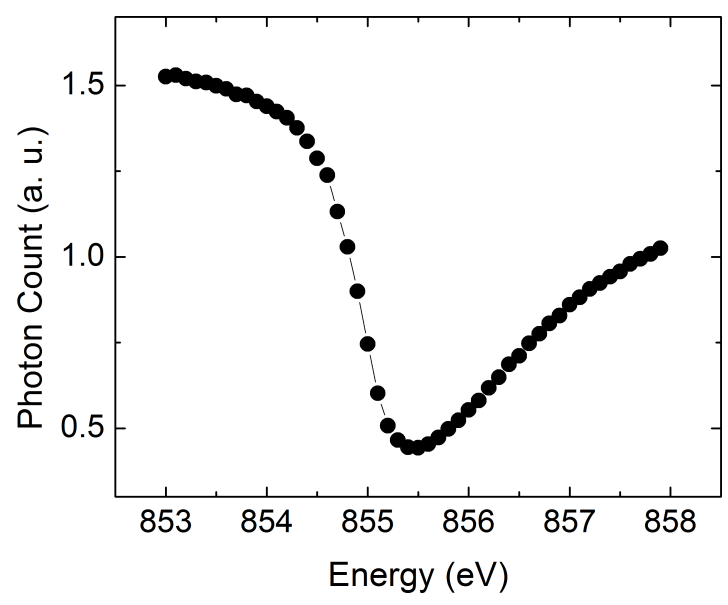
imaging. The zone plate focuses the monochromatic X-ray beam on to Figure 6.1: X-ray absorption spectra on a the sample and the transmitted X- $50 \mathrm{~nm}$ thick permalloy element. ray intensity through the sample is recorded pixel by pixel as the sample is scanned. Static images were deduced from the X-ray transmission signals for right and left circular polarization of the X-rays. The raw data obtained from the STXM is the transmitted intensity of X-rays at each pixel separated by a distance of about $10-15 \mathrm{~nm}$. The transmission intensity is depicted as: darker area means less photons (more absorption) are transmitted through the sample and brighter area means more photons (less absorption) are transmitted. We scan the same sample area with positive (negative) polarization of the X-rays and record the transmission images namely $I^{+}\left(I^{-}\right)$, as shown in Fig. 6.2 (a) and (b). The images recorded at opposite polarization are exploited to extract "dichroic image" [Fig. 6.2 (c)], using the following expression pixel by pixel:

$$
I^{X M C D}=\frac{I^{+}}{I^{-}}
$$

All individual patterns show regions with bright and dark contrast and in addition regions with gray contrast in between. Images with both polarizations of light, as expected, show an opposite magnetic contrast. As we can see from Fig. 6.2, the magnetic contrast is weaker than the topography contrast, thus even a small drift between images can cause artifacts in the dichroic images. To reduce the artifacts caused by a slow thermal drift, image alignment is necessary to correct for the drifts of the field of view. The alignment of images usually requires the existence of sharp and static features in the scanned area. In our case, we scanned the whole sample so that edges of the sample can be used to align the two images to get a dichroic 


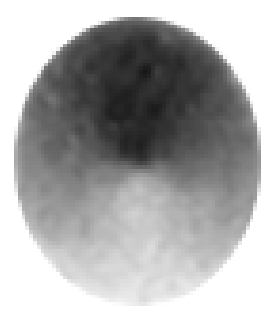

(a) $I^{+}$

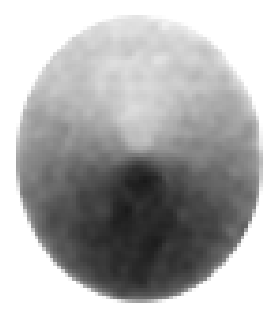

(b) $I^{-}$

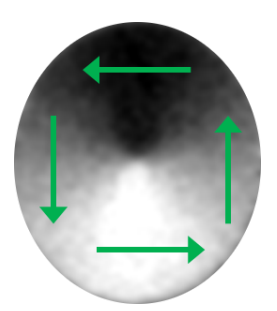

(c) $I^{+} / I^{-}$

Figure 6.2: STXM images of a disk (diameter-1.6 $\mu \mathrm{m}$ ) sample tilted at an angle of 30 degree to the photon propagation direction. The images are taken with positive (a) and negative (b) polarizations of the X-rays. A dichroic image (c) is deduced by dividing the two images.

image. This method improves the signal-to-noise-ratio, compared to the contrast in the individual images. The common topography component is normalized out and the magnetization component is enhanced. The black contrast reflects a parallel alignment between the magnetization direction and the photon propagation direction, while a white contrast represents an antiparallel alignment. Thus, the in-plane magnetization direction or circulation of the vortex structures can be derived from the magnetic contrast of these images. Static magnetic images deduced from X-ray transmission signals confirm a flux closed pattern of the inhomogeneous magnetization in such micron size magnetic elements. The arrows in Fig. 6.2(c), represents the magnetization direction which shows a CCW orientation of in-plane magnetization.

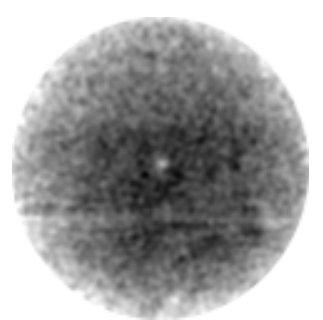

(a) $I^{+}$

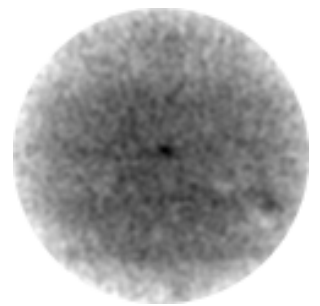

(b) $I^{-}$

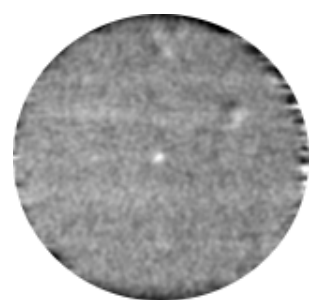

(c) $I^{+} / I^{-}$

Figure 6.3: STXM images of a disk sample of diameter $1.6 \mu \mathrm{m}$, mounted perpendicular to the photon propagation direction. A dichroic image (c) is deduced by dividing the two images taken with positive (a) and negative (b) polarization of the X-rays. The vortex core is visible as a bright or dark spot at the center of the disk. 
Out-of-plane: As only the out-of-plane magnetization contributes to the magnetic contrast, it is possible to image and derive the vortex core polarization directly in static mode by mounting the sample perpendicular to the photon propagation direction. Figure 6.3 shows the static magnetic configuration in a Permalloy disk of diameter $1.6 \mu \mathrm{m}$ and thickness of $50 \mathrm{~nm}$, where we can visualize the polarization of the vortex core. Images were taken for positive (Fig. 6.3 (a)), and negative (Fig.6.3 (b)) polarization of the X-rays. A dichroic image, shown in Fig. 6.3 (c), was deduced from both images, using relation (6.1). The adopted method is hereby sensitive only to the out-of-plane component of the magnetization. The image does not reveal much contrast but the vortex core is visible as a bright (dark) spot at the center of the disk.

\subsubsection{Dynamic Imaging}

The magnetic contrast depends also on the size of the magnetic feature due to the convolution with the spatial profile of the X-ray spot. As the vortex core has a size of about $10-20 \mathrm{~nm}$ only, it is not easy to detect the polarization of the core in static images and also the visible contrast could be due to a defect presented on that location. Therefore, imaging the dynamics of the vortex core is a better alternative to visualize its polarization quickly and with more certainty. We investigate the dynamics of a magnetic vortex confined in micron size magnetic elements (disk and square) by employing time-resolved scanning transmission Xray microscopy. The presence of specific excitation modes in thin film magnetic structures with a single vortex was mentioned in Chapter-2. One of the modes corresponding to the gyrotropic motion of the vortex core was observed in response to an in-plane oscillating magnetic field and current.

Pulse Excitation: We utilized a pulsed excitation technique to determine the resonance frequency of the magnetic element under investigation. A magnetic vortex in a square element of $1.6 \mu \mathrm{m}$ edge length was excited by the spin-transfer-torque effect. According to the micromagnetic simulations

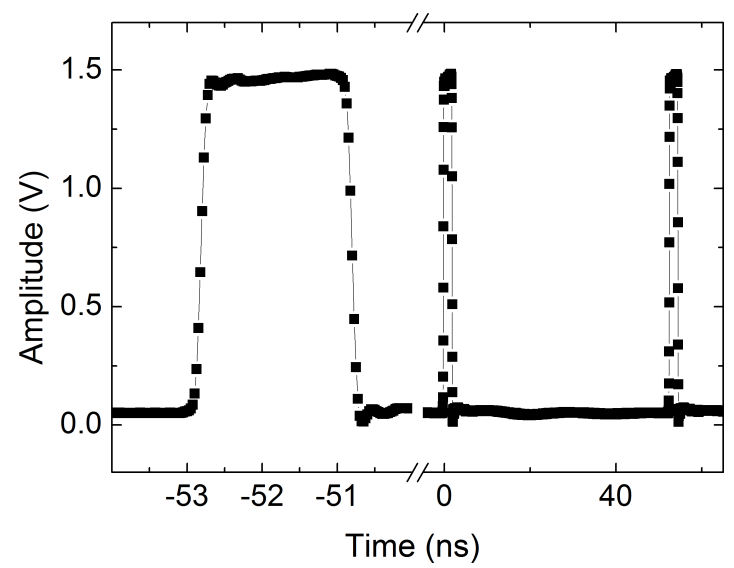

Figure 6.4: Pulse pattern. 


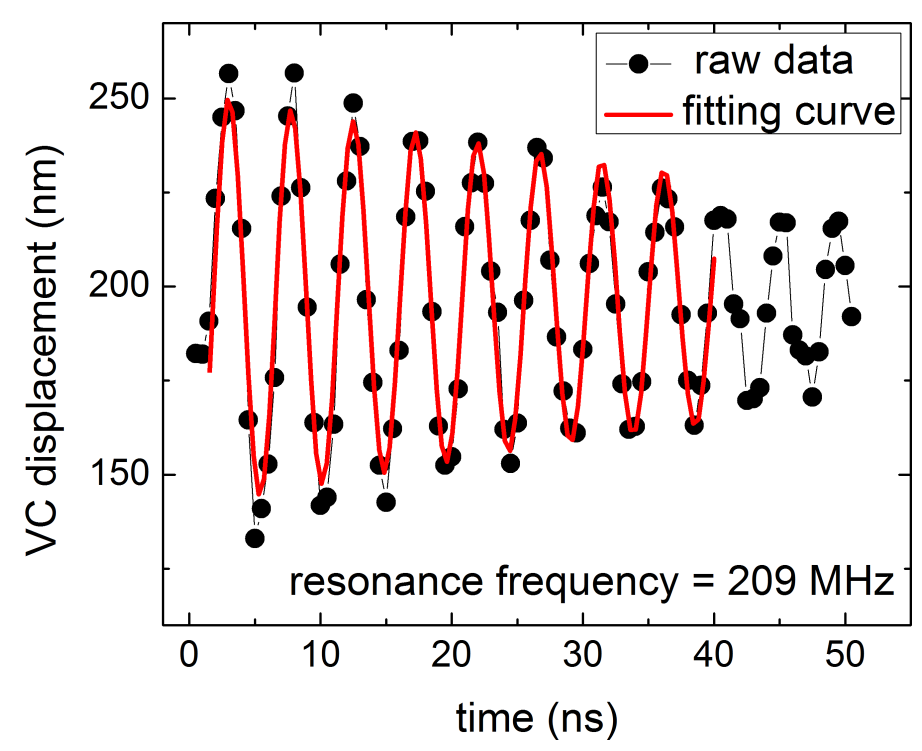

Figure 6.5: Displacement of the vortex core (VC) in x-direction is shown after the pulse excitation.

the expected gyro-frequency for this element is about $200 \mathrm{MHz}$ which corresponds to a time period of $5 \mathrm{~ns}$. For the exact determination of the gyro-frequency for this element the vortex core dynamics is excited by monopolar current pulses (Fig. 6.4) with pulse frequency of $19.33 \mathrm{MHz}$ (time period of $50 \mathrm{~ns}$ ) and width of $2 \mathrm{~ns}$. The time period of the pulse is sufficient to allow the vortex core to complete more than one gyration around its equilibrium position before it encounters the next pulse. When a current pulse reached the vortex core, it is pumped with energy for $2 \mathrm{~ns}$ which displaces the vortex core away from the equilibrium position. After the pulse, the vortex core moves on a spiral path around the equilibrium position with a pitch depending on the damping of the material. The displacement of the vortex core in $\mathrm{x}$-direction is plotted with time in Fig. 6.5. The curve in Fig. 6.5 shows the typical behaviour of a damped harmonic oscillator. Thus we can deduce the resonance frequency by fitting this curve with the following equation:

$$
y=y_{0}+A e^{-k_{r} t} \cos (2 \pi f t+\varphi)
$$

Here decay constant $k_{r}$ is related to the damping parameter of the material. The resonance frequency comes out to be $209 \mathrm{MHz}$ which is in good agreement with that found by micromagnetic simulations, see AMR simulations in Chapter-3.

Continuous Harmonic Excitation: The vortex core dynamics can also be induced by an in-plane oscillating magnetic field or current. In this case, the vortex core gyrates on a fixed orbit with the frequency of the excitation. This gyrotropic 


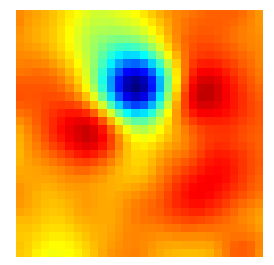

(a) $\mathrm{t}=0.00 \mathrm{~ns}$

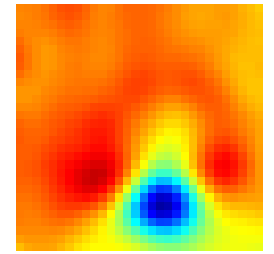

(f) $\mathrm{t}=2.70 \mathrm{~ns}$

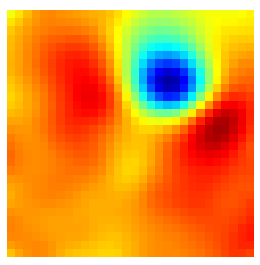

(b) $\mathrm{t}=0.54 \mathrm{~ns}$

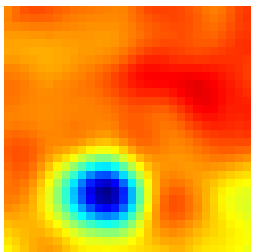

(g) $\mathrm{t}=3.24 \mathrm{~ns}$

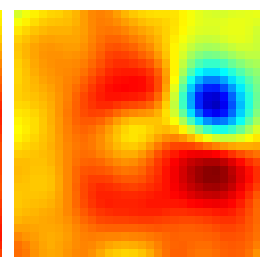

(c) $\mathrm{t}=1.08 \mathrm{~ns}$

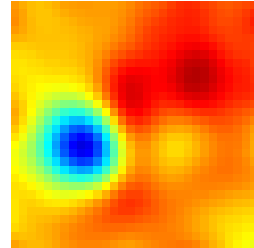

(h) $\mathrm{t}=3.78 \mathrm{~ns}$

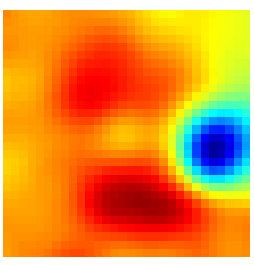

(d) $\mathrm{t}=1.62 \mathrm{~ns}$

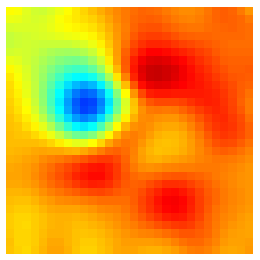

(i) $\mathrm{t}=4.32 \mathrm{~ns}$

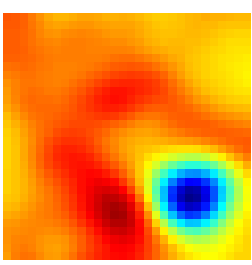

(e) $\mathrm{t}=2.16 \mathrm{~ns}$

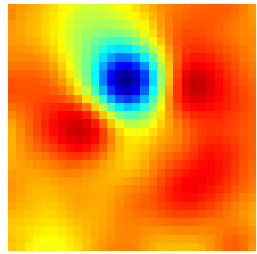

(j) $\mathrm{t}=4.86 \mathrm{~ns}$

Figure 6.6: Gyrotropic motion of the vortex core in a square of edge length $1.6 \mu \mathrm{m}$ excited with an rf current with a frequency of about $207 \mathrm{MHz}$ and amplitude of 2.8 $m A$. STXM images of the inner part (300 $\mathrm{nm} \times 300 \mathrm{~nm}$ ) of the magnetic element showing the position of the vortex core (blue) at different times.

motion can easily be induced with low excitation amplitude by tuning the excitation frequency " $f$ " near the resonance frequency of the vortex core. Figure 6.6 shows the normalized images of a moving vortex at different times where the blue spots represent the vortex core. The scanned area has a edge length of $300 \mathrm{~nm}$. In these images the sample is excited with an ac current with a frequency of 207 $\mathrm{MHz}$, near to the resonance frequency determined by the pulse excitation method. Our photon counting system distributes every other transmitted X-ray pulse to different photon counters, so that we record snapshots of the sample magnetization dynamics at equally distributed phases. In terms of time, these images are about 0.54 nanosecond apart. The magnetic images recorded by photon counters are sorted to the right phase order. A movie made out of these images clearly shows that the vortex core is gyrating in the clockwise direction on an orbit around the equilibrium position.

The radius of gyration of the vortex core is determined from these dynamic images. Figure 6.7 shows the gyrotropic radius calculated for different frequencies of the excitation current. Here we can clearly see a resonance curve with resonance frequency of about $207 \mathrm{MHz}$ which is also in agreement with that measured with pulsed excitation (Fig. 6.5). 


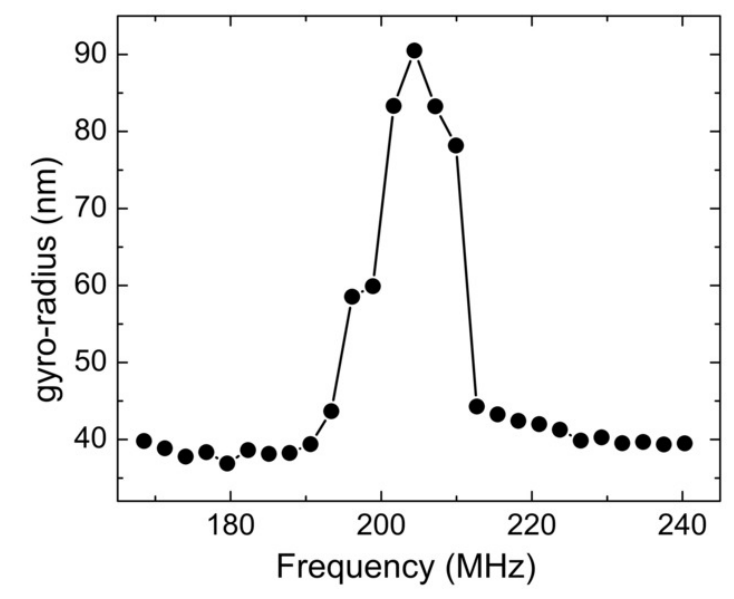

Figure 6.7: Resonance curve for a square element of edge 1.6 $\mu \mathrm{m}$ : The gyroradius measured in STXM is plotted for several excitation frequencies of the rf current.

\subsection{AMR Measurements}

\subsubsection{AMR}

A four terminal transport measurement technique is used to characterize the dc AMR behaviour of the sample by flowing a constant current of $50 \mu \mathrm{A}$ through the magnetic sample. An external in-plane dc magnetic field is varied between - 80 $\mathrm{mT}$ and $80 \mathrm{mT}$ and the voltage drop across the magnetic sample is measured in a nanovoltmeter. AMR curves for a disk and square sample made of Permalloy with lateral dimensions of $1.6 \mu \mathrm{m}$ and $50 \mathrm{~nm}$ thickness are shown in Figs. 6.8(a), (b) where the resistance (voltage drop across the sample divided by the current) is plotted for different strengths of the external dc magnetic fields.

Initially, we applied a dc magnetic field of $-80 \mathrm{mT}$ along the $\mathrm{X}(\mathrm{Y})$ direction that is sufficient to saturate the magnetic element to bring it in a single domain state by aligning the individual magnetic moments along (perpendicular to) the current flow direction. At higher magnetic fields, we can see that due to the AMR effect the resistance of the sample is higher for magnetic field applied parallel to the current flow direction (0 degree, $H \| I$, red line) compared to the perpendicular case (90 degree, $H \perp I$, black line). The Zeeman energy changes while varying the strength of the external magnetic field. When the Zeeman energy becomes sufficiently small, the magnetization curls along the edges of the microstructures to minimize stray fields, leading to a flux closure arrangement. As the field strength is reduced the resistance of the sample changes which indicates a change in magnetization state of 


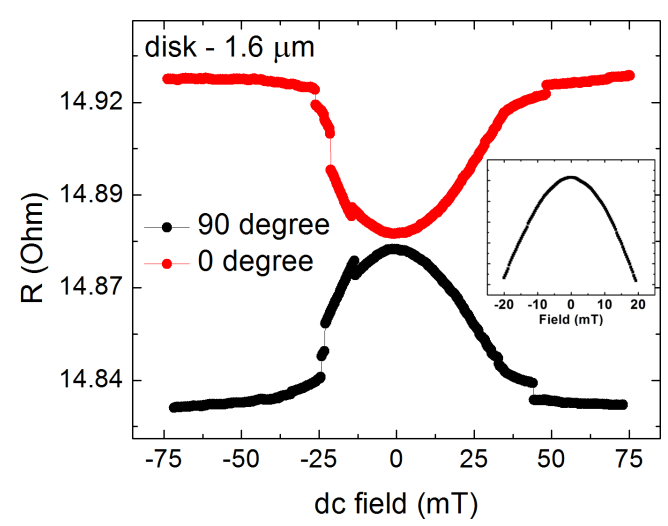

(a) dc AMR - disk

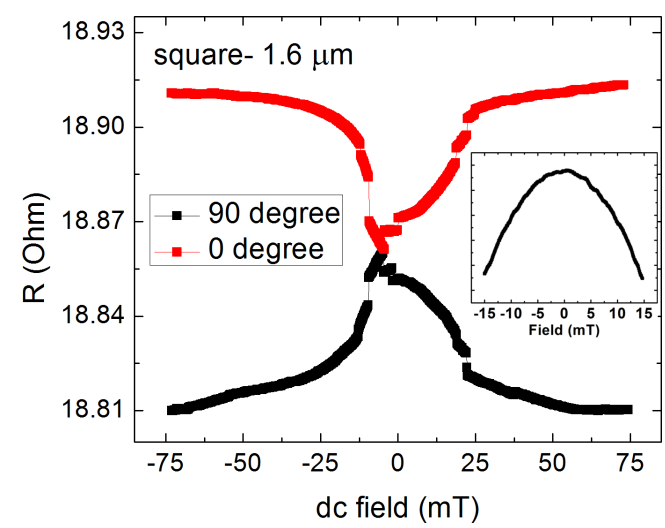

(b) dc AMR - square

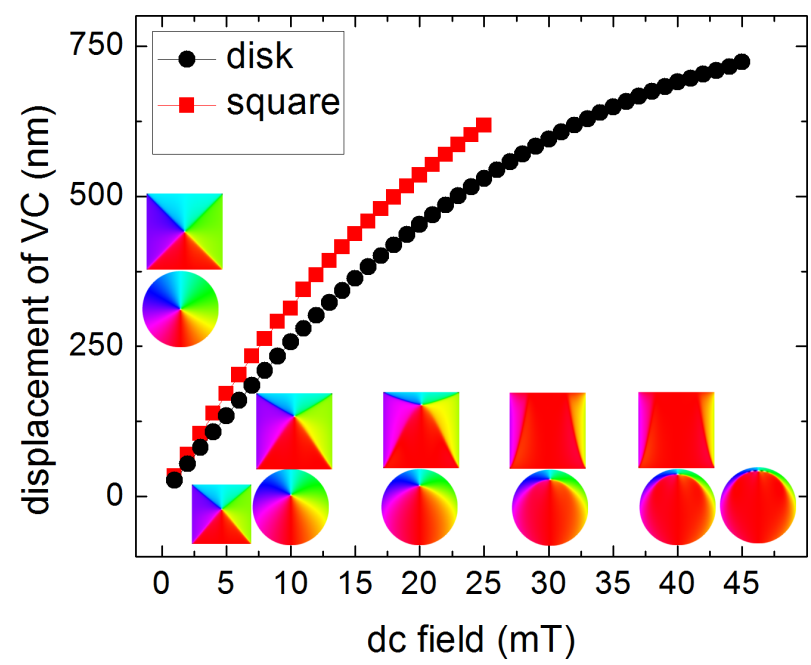

(c) VC position (simulations)

Figure 6.8: Experimentally measured dc-AMR curves for a disk (a) and a square shaped element (b). Micromagnetic simulation results for the position of the vortex core as a function of an external applied dc magnetic field (c) which confirms the annihilation fields of the vortex core for disk and square element as $45 \mathrm{mT}$ and 25 $m T$, respectively.

the sample. At a magnetic field of about $-14 \mathrm{mT}$ in case of the disk and $-5 \mathrm{mT}$ in case of the square, there is a small kink in the resistance owing to the nucleation of the vortex core in the micro magnetic elements. The vortex core reaches the center of the element at zero field resulting in a maxima/minima in the AMR curves. Now increasing the field strength in the other direction moves the vortex core away from the center of the element. After a certain value of the external field, indicated by small but sharp jumps at positive fields $(+45 \mathrm{mT}$ for the disk and $+25 \mathrm{mT}$ for 


\subsection{AMR Measurements}

the square), the vortex core escapes from the samples at the so-called annihilation field where the magnetic element transforms back into a single domain or into an onion state. After this point, there is no significant change in the voltage signal which indicates the saturation state in the other direction. The AMR curves show almost reversible non-symmetric curves and different magnetization processes can be investigated with these curves such as: the nucleation, the propagation and the annihilation of the vortex core. If we sweep the magnetic field in a small range below the annihilation field e.g. from $-15 \mathrm{mT}$ to $+15 \mathrm{mT}$, the vortex core is displaced away from the center of the sample symmetrically for positive and negative fields and the observed signals are more symmetric around zero field as shown in the inset of respective graphs in Fig. 6.8 a) and b). The annihilation fields are also confirmed by micromagnetic simulations by calculating the position of the vortex core while sweeping the external magnetic field at a rate of 1 Oe/ns, see Fig. 6.8 c). Here we notice that the vortex state does not exist for field strengths larger than $25 \mathrm{mT}$ for square and $45 \mathrm{mT}$ for disk shaped element.

\subsubsection{Homodyne Detection}

The measurements presented in this section are based on the previously mentioned versatile and phase sensitive transport measurement technique the so called "Homodyne detection technique", in which the microwave current gets rectified due to the symmetry breaking of the system by an externally applied static magnetic field. By tuning the strength and direction of the externally applied dc-field we can move the vortex core away from the center of the magnetic element. To protect the sample from rapid field jumps which could cause the switching of the vortex core and could enhance the noise level, the magnetic field was increased in small steps (1 2 Oe) which provides a smooth transition from one field to the other. Contrary to the other measurement techniques, the homodyne detection scheme does not need any external DC source and the rectified signal can directly be measured in a nanovoltmeter via the dc port of the bias-tee.

The rectified homodyne voltage signal, due the anisotropic magnetoresistance in the magnetic element, is measured as a function of the excitation frequency $(f=\omega / 2 \pi)$ in the vicinity of the resonance frequency of the vortex core. However some care needs to be taken into account when dealing with microwave circuits. A microwave is an electromagnetic wave which has difficulties to pass through junctions because of impedance mismatch. In reality we can not avoid junctions in our devices which could cause a problem to pass the microwave signal through the 
sample. The impedance mismatch induces problems specially for the frequency sweep measurements as the intensity of the transmitted signal depends upon the frequency. Therefore a normalization of the experimentally measured signal is necessary to reduce the intrinsic noise and background effects to get a better signal to noise ratio. It has already been explained in Chapter-3 that due to the symmetry there is no dc component in the homodyne signal for zero external applied dc-field. We took advantage of this fact to normalize the data by subtracting the signal at zero field from the raw data.

Figure 6.9 shows the measured rectified resonance spectra of the AMR signal generated from the gyrotropic motion of the $\mathrm{VC}$ as a function of bias fields for three different excitation currents $[1 \mathrm{~mA}$ in (a), $2 \mathrm{~mA}$ in (b) and $4 \mathrm{~mA}$ in (c)]. We observe that a large AMR signal appears near $210 \mathrm{MHz}$ which corresponds to the resonance frequency of the element as measured in STXM, see Fig. 6.7. With increasing external field the asymmetry (e.g. of the Landau domain structure) increases and hence the amplitude of the AMR signal also increases as shown in the inset of Fig. 6.9(c). Moreover, some other important phenomena are observed by analyzing the homo-

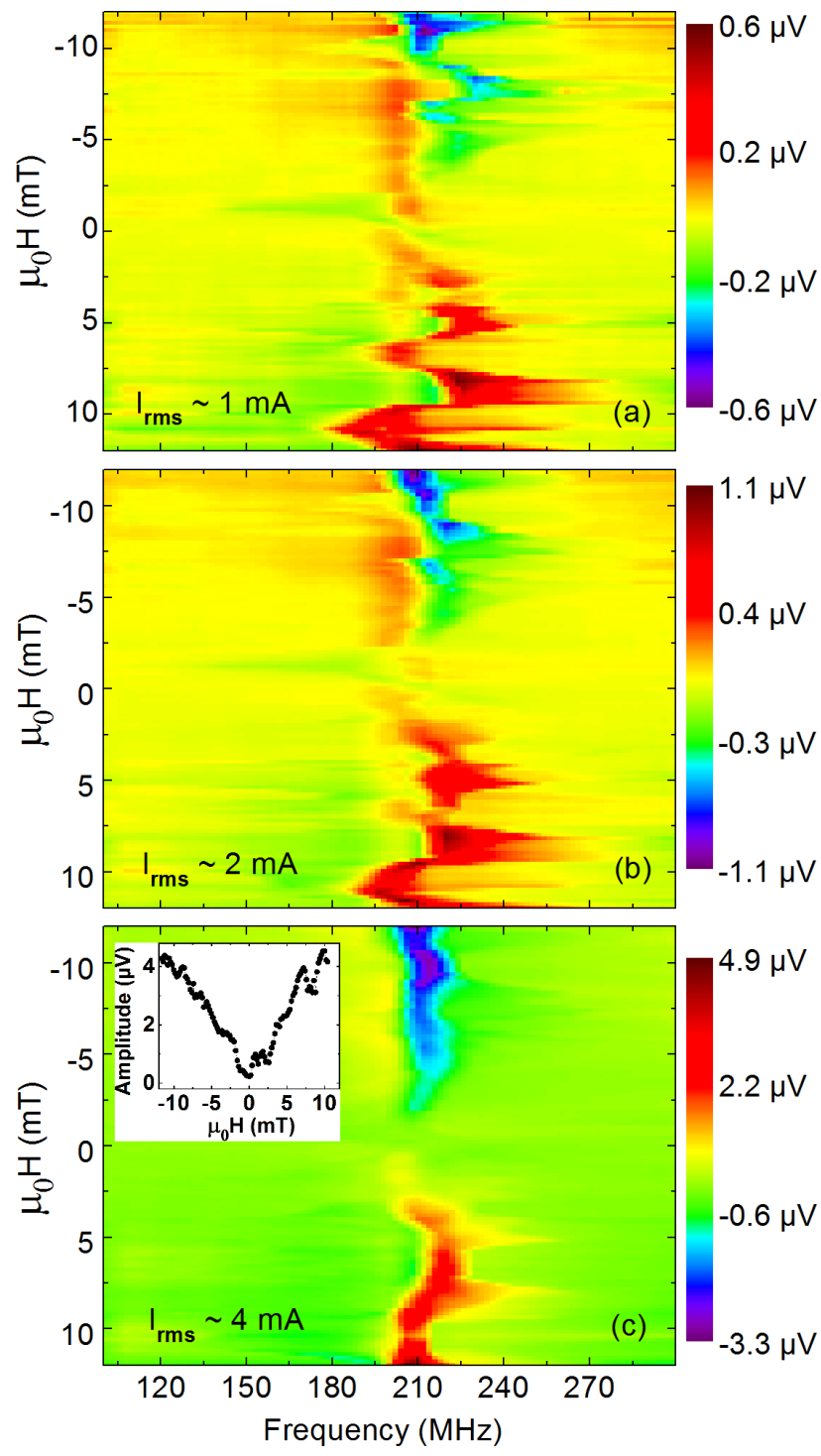

Figure 6.9: AMR spectra measured on a square shaped sample for three different excitation currents (1 $m A$ in a) $2 m A$ in b) and $4 m A$ in $c))$. The inset in c) shows the amplitude of the AMR signal at $4 \mathrm{~mA}$, as a function of bias field. 


\subsection{AMR Measurements}

dyne signals. Our power dependent measurements reveal that the dynamics of a vortex core can also be affected by local pinning sites. We observe random variations in the resonance frequency with increase in the external bias field. The low and nearly constant values of the resonance frequencies $\left(f_{R}\right)$ are actually the behaviour expected from the quasiharmonic magnetostatic geometric potential and can also be well reproduced by micromagnetic simulations but random peaks in $f_{R}$ are unexpected. The observed deviations of the resonance peak position as a function of bias field can be attributed to local modifications of the magnetic properties of the sample since the gyrating vortex core is moved across the sample when applying the external magnetic field, see ref. [125, 126, 127]. These modifications could arise from surface roughness or from local pinning sites and defects present in the sample. The randomness in resonance frequencies is more pronounced for low excitation power, as we can see in (Fig. 6.9). With increasing power the gyro-radius increases and the effect of random local deviations thus averages out on the longer gyro-trajectory. Thus the gyrating VC can be effectively used to investigate the magnetic inhomogeneity of the samples [125, 126].

In order to be able to investigate the AMR signal for the two polarities of the vortex core a disk shaped sample of $1.6 \mu \mathrm{m}$ diameter and $50 \mathrm{~nm}$ thickness with four symmetric contacts is used (Fig. 6.10). In this configuration, the VC polarity can be switched selectively to the up (down) state by a counter-clockwise (clockwise) rotating current. The vortex core with up $(p=+1)$ polarity rotates in the counterclockwise direction and the $\mathrm{VC}$ with down $(p=-1)$ polarity in the clockwise direction. Therefore, a phase difference of $180^{\circ}$ occurs between the dynamics of the $\mathrm{VC}$ with the two opposite polarities which results in a sign change of the homodyne voltage signal as described in Chapter-3. Figures 6.10(a) and (b) clearly shows a sign reversal in the AMR spectra for the two polarities of the vortex core and also the observed fine structures in the spectra remain unchanged when the $\mathrm{VC}$ polarity is reversed. Additionally, we notice a significant shift of the resonance frequency with bias fields [128] which is not so pronounced in the case of the square element. This indicates that the magnetization in the disk shaped sample is becoming stiffer with increasing external field. Our micromagnetic simulations confirm such an increase in the resonance frequency, see Fig. 6.11(a). The vortex core gyrates around an equilibrium position, which shifts through the disk as the magnetic field is swept. The shifting of the vortex core position is connected to the change in curvature of the potential in which the vortex core moves. This change in curvature reflects in the observed frequency shift with applied magnetic field. 


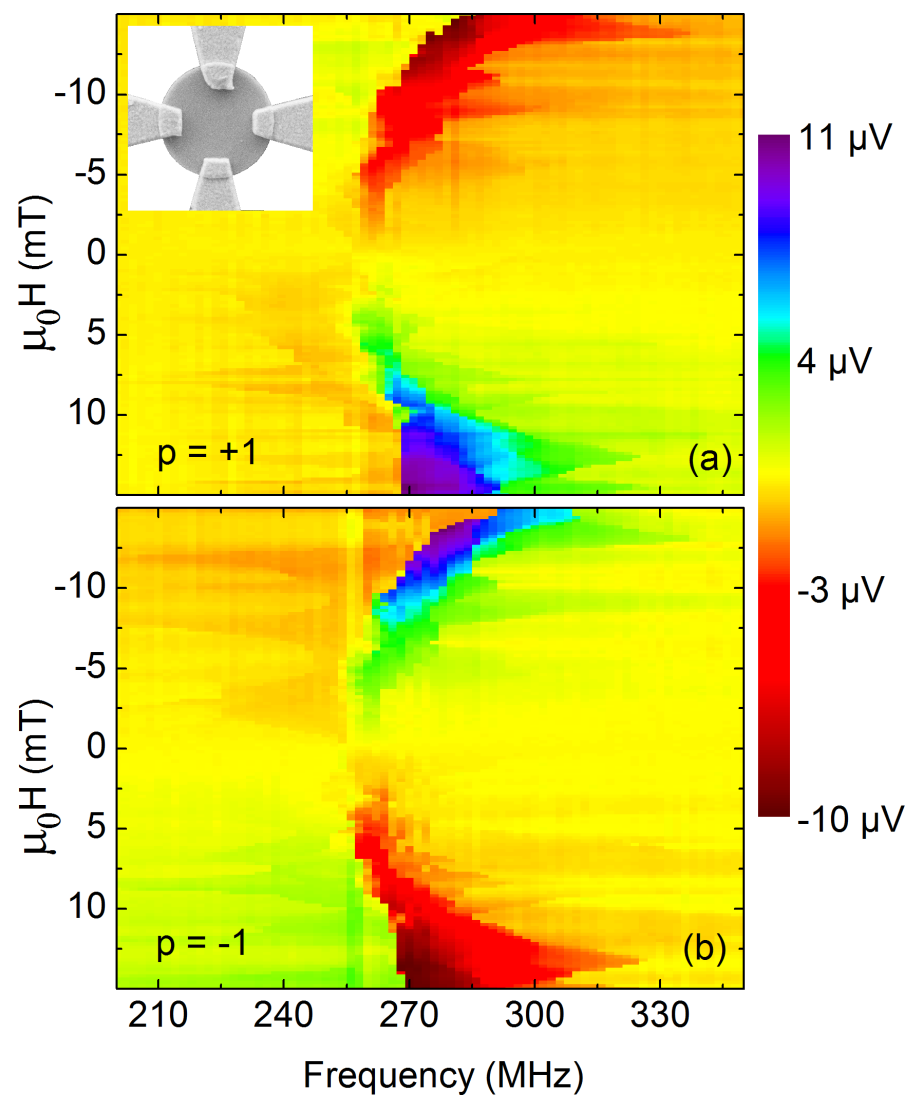

Figure 6.10: AMR spectra measured on a disk shaped sample for a) up $(p=+1)$ and $b)$ down $(p=-1)$ polarization of the vortex core for an excitation current of 1.2 $m A$. By applying a rotating current (counterclockwise or clockwise) the VC polarity can be switched selectively to up or down. A sign change of the AMR voltage signal can be observed for the two polarities. Note that a significant shift of the resonance frequency to higher frequencies as we increase the bias field. Also note that the fine structure observed in the spectra does not depend on the VC polarity.

We have performed more micromagnetic simulations to understand this shift in the resonance frequency in more detail. As the minimum energy state of such elements in the vortex state is when the vortex core lies at the center of the element, the displacement of the vortex core away from the center causes an increase in energy of the magnetic system. An in-plane external dc magnetic field is applied to move the vortex core away from the center of the magnetic element and the energy (excluding the Zeeman energy) in the magnetic element is calculated for different positions of the vortex core. In Fig. 6.11 (b) we have plotted the average energy of the magnetic system normalized with that in zero external magnetic field. As we can see, the average energy of the magnetic element increases with the displacement 

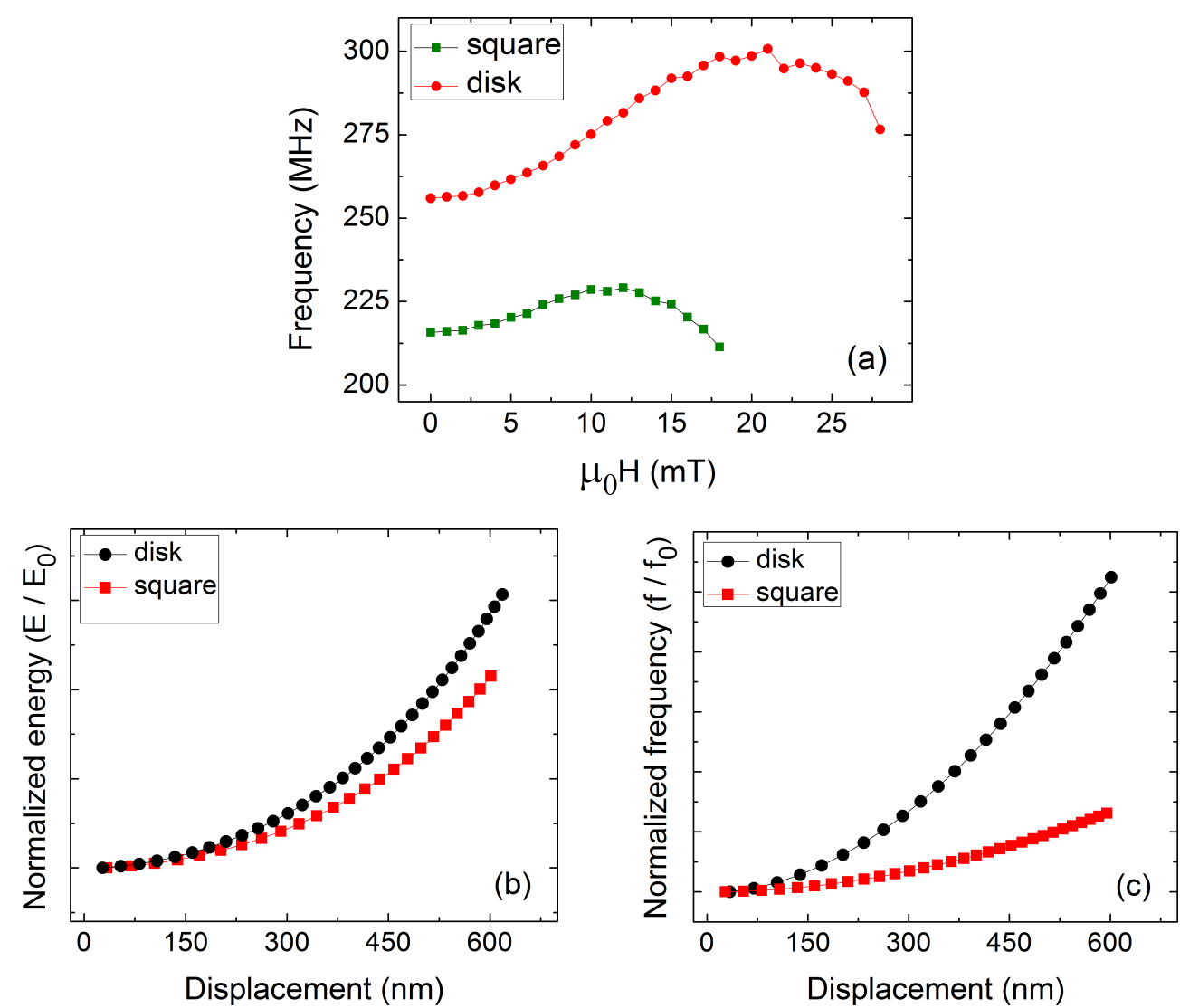

Figure 6.11: Micromagnetic simulation results for a disk and square element. (a) Resonance frequencies calculated directly by exciting the vortex core by a magnetic field pulse at different values of the bias fields. (b) Normalized average energy of the sample and (c) the resonance frequencies proportional to the double derivative of the average energy as a function of the displacement of the vortex core.

of the vortex core away from the center. Now we fit this energy curve with a fourth order polynomial and calculated its double derivative which is proportional to the resonance frequency of the vortex core. Figure 6.11 (c) shows the normalized frequency plotted for different positions of the vortex core and confirms an increase in resonance frequency as a result of the increase in magnetostatic energy with the position of the vortex core (or with external magnetic field). This increase in energy can be attributed to the formation (in the case of a disk) and/or sharpening (in the case of a square) of the domain walls as shown in Fig. 6.12. In the case of a square element (see Fig. $6.12(\mathrm{e}-\mathrm{h})$ ), domain walls already exist and therefore the increase in energy is less than that in the case of a disk [Fig. 6.11 (a)]. 


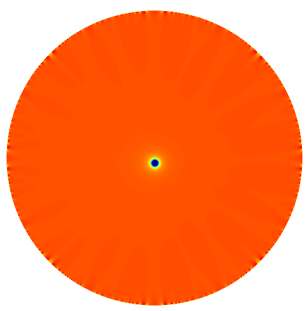

(a) $0 \mathrm{mT}$

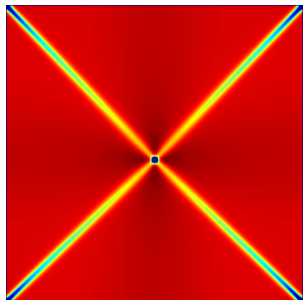

(e) $0 \mathrm{mT}$

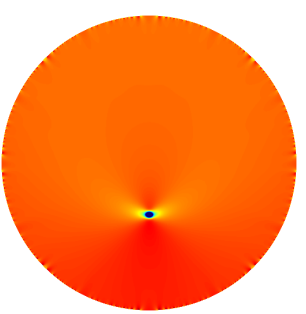

(b) $10 \mathrm{mT}$

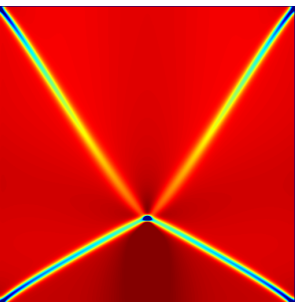

(f) $10 \mathrm{mT}$

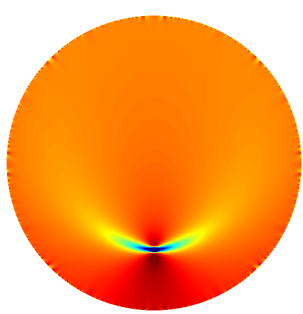

(c) $20 \mathrm{mT}$

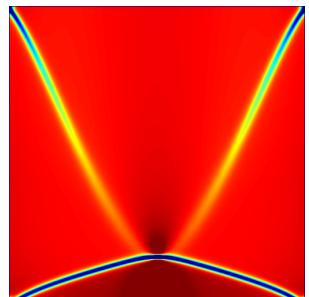

(g) $20 \mathrm{mT}$

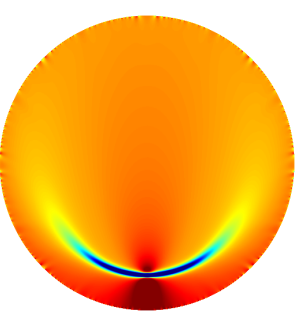

(d) $30 \mathrm{mT}$

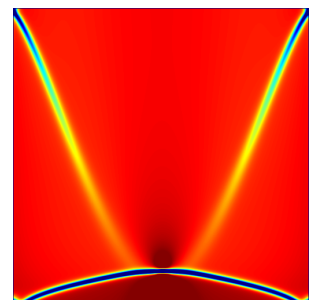

(h) $24 \mathrm{mT}$

Figure 6.12: Energy distribution for different positions of the vortex core. Blue high energy density; Red - low energy density.

\subsection{Other Experiments}

As described in chapter-4, several types of samples are prepared for different experiments. The results of those experiments are summarized below.

- Spin-Transfer-Torque Induced Vortex Dynamics: The magnetization dynamics in a disk sample (Fig. 4.4) was excited with rotating rf currents with frequencies in sub-GHz and multi-GHz range. It has been found that, similar to the excitations with rotating magnetic fields [61, 69, 70, 71, 72], the vortex core polarity can be switched selectively by STT excitations due to rotating electrical currents (frequency: sub-GHz) when the sense of rotation (CW or $\mathrm{CCW}$ ) of the vortex core is matched with that of the rotating current. Also for the $\mathrm{VC}$ switching with multi-GHz rotating currents, not only the eigenfrequency but also the sense of rotation have to be matched with the specific spin wave eigenmode. (Experiments have been performed by Markus Sproll [78]).

\section{- Interaction Between the Vortex Gyromode and Azimuthal Spin}

Waves: A different approach is used to reverse the vortex core polarity by simultaneious excitation with (multi-GHz) azimuthal spin waves and the (sub$\mathrm{GHz}$ ) vortex gyromode in a disk sample (Fig. 4.5). It is shown that an active 
dual frequency excitation of both the sub-GHz vortex gyromode and multi$\mathrm{GHz}$ spin waves considerably changes the frequency response of spin wave mediated vortex core reversal. Besides additional minima in the switching threshold, a significant broadband reduction of the switching amplitudes is observed, which can be explained by non-linear interactions between the vortex gyromode and the spin waves. (Experiments have been performed by Markus Sproll [78, 80]).

- Unidirectional sub-100 ps Vortex Core Reversal with Short Magnetic Field Pulses: The vortex core, in a ferromagnetic disk of diameter $500 \mathrm{~nm}$ (Fig. 4.5), is excited with a sequence of two orthogonal monopolar magnetic pulses, less than $100 \mathrm{ps}$ in total duration. The fact that digital pulses can be used for fast spin wave mediated switching instead of rotating fields is an attractive aspect for potential technological applications. The phase diagram of vortex core reversal is measured, i.e., the dependence of switching on pulse amplitudes and their durations. A region of unidirectionality is found to be rather large and thus robust against sample dimension variations. The finding that the region of unidirectionality is larger than the corresponding region for rotating field bursts is a further important result. The measurements indicate a fast switching time below 100 ps which is in agreement with the micromagnetic simulations. The coupling between spin waves and the vortex gyromode is identified as the origin of the asymmetric response of the magnetization to clockwise (CW) or counterclockwise (CCW) excitation which is responsible for the unidirectionality in the vortex core reversal by this broadband orthogonal short pulse excitation. (Experiments have been performed by Matthias Noske [91]).

- Coupled Magnetization Dynamics and Reversal in GdFe/Al/Py Stack:

Additional layers of $\mathrm{Al}$ and GdFe are grown on top of the permalloy disk (Fig. 4.5). It is demostrated that GdFe with perpendicular anisotropy can enhance the vortex core magnetization. It has been observed that switching of the vortex core polarization of the permalloy layer causes a magnetization reversal of the complete GdFe layer and thus significantly increases the magnitude of switched magnetization. Therefore, GdFe magnifies the vortex core polarization and thus could be measured easily e.g. by GMR sensors compare to reading a single vortex core of the size of only about $10-20 \mathrm{~nm}$ in diameter. (Experiments have been performed by Georg Dieterle). 
- Non-Linear Radial Spin Wave Modes in Thin Magnetic Disks: Figure 4.7 shows the sample used to study radial spin wave modes in thin Permalloy platelets in vortex state. The thickness was kept thin so the onset of nonlinear behavior can be observed at relatively low excitation strengths, as it was found from micromagnetic simulations. The spin wave amplitude was measured using a frequency resolved magneto optical network analyser [129] which allows us to record high resolution resonance curves. It was found that with increasing excitation amplitude up to about $10 \mathrm{mT}$, the lowest order spin wave mode behaves strongly non-linearly as the mode frequency red-shifts and the resonance peak strongly deforms. At higher excitations, the spin waves are transformed into a soliton by self-focusing and collapse onto the vortex core, dispersing the energy in short-wavelength spin-waves. This process can also lead to switching of the vortex polarization through the injection of a Bloch point [64]. (Experiments have been performed by Mathias Helsen [84, 129]).

- Propagation of Spin Waves in an Antidot Lattice: Propagation of spin waves in an antidot lattice (Fig. 4.8) is investigated by direct observation in a STXM. The introduction of the artificial periodicity allows to alternate the spin wave dispersion in the material and to form a spin wave guide or filter. Magnon modes spanning from $250 \mathrm{MHz}$ up to $8 \mathrm{GHz}$ were resonantly excited and the influence of a variable applied field was investigated. These measurements allowed the direct observation of the individual modes, their interaction with the antidot lattice, and their respective localisation within the lattice. Furthermore, these measurements indicate that these mechanisms in magnonic crystals can be exploited to focus magnons laterally. (Experiments have been performed by Joachim Gräfe). 
Magnetization dynamics in ferromagnetic samples is discussed with special emphasis on micron and submicron size platelets in the vortex ground state. Magnetization dynamics in vortex structures has been investigated under the influence of magnetic fields and currents. It is shown that the vortex core starts to gyrate around its equilibrium position if the system is continuously pumped with sufficient energy via oscillating magnetic fields or currents. The magnetization distribution and dynamics in these vortex structures is directly observed in a spatial and time resolved technique - scanning transmission X-ray microscopy. It is necessary to find a way to read the four degenerate ground states of these structures for its implementation in future technological devices. We have utilized a well known phenomenon the so called anisotropic magnetoresistance effect to complete the task of reading these vortex states electrically. Due to the gyrotropic motion of the vortex core, excited with the spin-transfer-torque effect by flowing an rf current though it, the magnetization and hence the resistance of the samples changes periodically. The frequency of resistance oscillation is twice the frequency of the excitation current as the resistance of the sample is same for the symmetric positions of the vortex core around the center of the magnetic element which can be seen in dc AMR measurements, Fig. 6.8. This periodic change in resistance together with the rf current gives rise to a periodic homodyne voltage signal across the sample. This homodyne signal has been rectified by introducing an asymmetry using an in-plane static magnetic field. In this case the gyrotropic motion of the vortex core is not symmetric around the center of the structure anymore. This gives rise to a resistance component which oscillates with the frequency of the excitation current and gives us a rectified homodyne voltage signal. This signal is measured for several frequencies of the excitation current across the expected resonance frequency of the magnetic element. The same signal is measured for the two polarities of the vortex core and it has been observed that the sign of the signal changes with change of polarity. Thus the polarization state of the vortex core can be determined by this technique. If we change the direction of the bias field (or the circulation of the vortex structure) the vortex core is displaced in the other direction and we observed a sign reversal 
of the measured AMR signal. Therefore, there is a sign reversal of the measured voltage signal if we change either the polarization or the circulation of the vortex structure. Alternatively and complementarily, micromagnetic simulations were also conducted to study the AMR effect in these structures. The results of our micromagnetic simulations are in very good agreement with our experimental results and thus support the validity of our technique. This technique can be a promising method to detect the vortex states easily if the vortex structures are implemented in future applications.

The phenomenon of spin-motive-force has also been investigated in the vortex structures by micromagnetic simulations. It has been found that this effect can also be utilized to determind the vortex state electrically but more care needs to be taken in this case. It is shown that if we measure the oscillating voltage signal generated due to the gyrotropic motion of the vortex core, there is a phase difference of 180 degree for the two circulation directions. Additionally, if the voltage probes are located along the direction of the excitation $\mathrm{rf}$ field, the phase of the SMF signal for the two polarities of the vortex core also differs by 180 degrees. Thus we can determine the vortex state by carefully examining the phase relation of the voltage signal for different polarization and circulation of the vortex core. Recently, the SMF signal generated due to the gyrotropic motion of the vortex core has been measured experimentally [112]. However, this measured signal could be due to the AMR effect (see eq. 3.1) which shows similar behaviour and produces a larger signal compare to that due to the SMF effect, calculated with micromagnetic simulations. An attempt has been made to measure the SMF signal experimentally but it has not been successful so far.

The goal of this project/thesis was to find a way to detect the vortex state in micromagnetic elements by electrical means so that these elements can be used in future technology. Much remains to be done before the four value multibit becomes practical. However these investigations have demonstrated the feasibility of these measurement techniques and could pave the way towards the implementation of magnetic vortices in future technological devices. 


\section{Publications}

1. M. Noske, H. Stoll, M. Fähnle, A. Gangwar, G. Woltersdorf, A. Slavin, M. Weigand, C. H. Back and G. Schütz. The third dimension: Hybridization of flexure gyromode with spin waves. Submitted.

2. A. Gangwar, Hans G. Bauer, J.-Y. Chauleau, M. Noske, M. Weigand, H. Stoll, G. Schütz and C. H. Back. Electrical determination of vortex state in submicron magnetic elements. Phys. Rev. B. 91, 094407 (2015).

3. M. Helsen, A. Gangwar, J. De Clercq, A. Vansteenkiste, M. Weigand, C. Back, and B. V. Waeyenberge, Non-linear radial spinwave modes in thin magnetic disks, Appl. Phys. Lett. 106, 073503 (2015).

4. M. Noske, A. Gangwar, H. Stoll, M. Kammerer, M. Sproll, G. Dieterle, M. Weigand, M. Fähnle, G. Woltersdorf, C. H. Back, G. Schütz, Unidirectional sub-100 ps magnetic vortex core reversal, Phys. Rev. B. 90, 104415 (2014).

5. M. Helsen, A. Gangwar, A. Vansteenkiste, and B. Van Waeyenberge, MagnetoOptical Network Analyser: A novel method to investigate nanoscale magnetical dynamics, Rev. Sci. Instrum. 85, 083902 (2014).

6. M. Sproll, M. Noske, H. Bauer, M. Kammerer, A. Gangwar, G. Dieterle, M. Weigand, H. Stoll, G. Woltersdorf, C. H. Back and G. Schütz, Low-amplitude magnetic vortex core reversal by non-linear interaction between azimuthal spin waves and the vortex gyromode, Appl. Phys. Lett. 104, 012409 (2014).

7. Rastogi, A., Kushwaha, A. K., Shiyani, T., Gangwar, A. and Budhani, R. C., Electrically Tunable Optical Switching of a Mott Insulator-Band Insulator Interface. Adv. Mater., 22, 4448, (2010) 


\section{Thiele Equation}

For a rigid vortex, the magnetization distribution can be described by the vortex core position $\vec{X}$. Thiele used Cartesian coordinates under the assumption of a rigid vortex under linear translations. The magnetization is then written as

$$
\vec{M}(\vec{x})=\vec{M}_{0}(\vec{x}-\vec{X})
$$

where $\vec{x}$ is the space coordinate and $\vec{M}_{0}$ is the magnetization distribution when the vortex core is located at the origin (center of the magnetic element).

The work density done under a magnetic field is $d w=\vec{H} \cdot d \vec{M}$. Since the magnetization is a function of position, the equation can be written for the cartesian coordinate as

$$
d w=\vec{H} \frac{\partial \vec{M}}{\partial X_{i}} d X_{i}=f_{i} d X_{i}
$$

for summation over $i=x, y, z$ where the force density $f_{i}$ is given by

$$
f_{i}=\vec{H} \frac{\partial \vec{M}}{\partial X_{i}}=-\vec{H} \frac{\partial \vec{M}}{\partial x_{i}}
$$

Thus, we can write the gyrotropic and damping force density as

$$
\begin{gathered}
f_{i}^{g}=-\vec{H}^{g} \frac{\partial \vec{M}}{\partial x_{i}}=\frac{1}{|\gamma| M_{s}^{2}} \varepsilon_{l m n} M_{l} \frac{\partial M_{m}}{\partial x_{i}} \frac{d M_{n}}{d t} \\
f_{i}^{d}=-\vec{H}^{d} \frac{\partial \vec{M}}{\partial x_{i}}=\frac{\alpha}{|\gamma| M_{s}} \frac{\partial M_{n}}{\partial x_{i}} \frac{d M_{n}}{d t}
\end{gathered}
$$

using the rigid vortex assumption we can write,

$$
\frac{d M_{n}}{d t}=\frac{\partial M_{n}}{\partial X_{j}} \frac{d X_{j}}{d t}=\frac{\partial M_{n}}{\partial X_{j}} v_{j}=-\frac{\partial M_{n}}{\partial x_{j}} v_{j}
$$

We can use the equation above to simplify the expression for the gyroforce and damping force as following

$$
f_{i}^{g}=\frac{1}{|\gamma| M_{s}^{2}} \varepsilon_{l m n} M_{l} \frac{\partial M_{m}}{\partial x_{i}} \frac{\partial M_{n}}{\partial x_{j}} v_{j}=-\varepsilon_{i j k} v_{j} g_{k}
$$


where $g_{k}$ is termed as the gyrocoupling vector given by

$$
g_{k}=\frac{1}{2|\gamma| M_{s}^{2}} \varepsilon_{i j k} \varepsilon_{l m n} M_{l} \frac{\partial M_{m}}{\partial x_{i}} \frac{\partial M_{n}}{\partial x_{j}}
$$

and

$$
f_{i}^{d}=-\frac{\alpha}{|\gamma| M_{s}} \frac{\partial M_{n}}{\partial x_{i}} \frac{\partial M_{n}}{\partial x_{j}} v_{j}=d_{i j} v_{j}
$$

with the dissipation tensor

$$
d_{i j}=-\frac{\alpha}{|\gamma| M_{s}} \frac{\partial M_{n}}{\partial x_{i}} \frac{\partial M_{n}}{\partial x_{j}}
$$

The total force $F_{i}$ exerted on the vortex core is given by integrating the force density $f_{i}$ over the volume $\mathrm{V}$ of the sample. Thus, the total force terms become

$$
\left\{\begin{array}{l}
F_{i}^{g}=\int f_{i}^{g} d V=-\varepsilon_{i j k} G_{k} v_{j} \\
F_{i}^{d}=\int f_{i}^{d} d V=D_{i j} v_{j}
\end{array}\right.
$$

where the total gyrocoupling vector and the total dissipation tensor are given by

$$
\left\{\begin{array}{l}
G_{k}=\int g_{k} d V=\frac{1}{2|\gamma| M_{s}^{2}} \varepsilon_{i j k} \varepsilon_{l m n} \int M_{l} \frac{\partial M_{m}}{\partial x_{i}} \frac{\partial M_{n}}{\partial x_{j}} d V, \\
D_{i j}=\int d_{i j} d V=-\frac{\alpha}{|\gamma| M_{s}} \int \frac{\partial M_{n}}{\partial x_{i}} \frac{\partial M_{n}}{\partial x_{j}} d V
\end{array}\right.
$$

The force from the effective field $\vec{H}_{\text {eff }}$ originally appearing in the LLG equation can simply be given by the magnetic potential as

$$
\vec{F}_{e f f}=-\nabla U
$$

where the magnetic potential includes the exchange, anisotropy and magnetostatic energy and is given as a function of the vortex core position $\vec{X}$. Now we can convert the magnetic field equation-[2.5] into the force equation as

$$
\vec{F}_{e f f}+\vec{F}^{g}+\vec{F}^{d}=\vec{F}_{e f f}+\vec{G} \times \vec{v}+\vec{D} \cdot \vec{v}=0
$$

This is the force balance equation[19, 48, 83] originally described by Thiele in his paper to describe the vortex dynamics as a rigid particle. For a thin film geometry the total gyrotropic vector is perpendicular to the film plane, i.e. $\vec{G}=G \hat{z}$ if we put the film in (x,y) plane. The gyroconstant is $G=2 \pi p q t_{f} M_{s} / \gamma, t_{f}$ is the thickness. The topological charge $q= \pm 1, \pm 2, \ldots$ determines the direction of the vortex core 
rotation and its frequency. The vortex polarization $\mathrm{p}$ is determined by $M_{z}$, it is ascribed to the assumption of the uniform magnetization across the thickness i.e. $\frac{\partial M}{\partial z}=0$. From this assumption, the z-index components of the total dissipation tensor $\vec{D}$ vanishes i.e. $D_{x z}=D_{y z}=D_{z z}=D_{z x}=D_{z y}=0$. Together with this, for the vortex structure, the off diagonal components of the total dissipation tensor vanish i.e. $D_{x y}=D_{y x}=0$ and the diagonal elements have the unique value i.e. $D_{x x}=D_{y y}=D$ due to symmetry. Thus the total dissipation force can be written as $\vec{F}^{d}=\vec{D} \cdot \vec{v}=D \vec{v}$ where $\vec{v}$ lies in the (x,y) plane. The energy function is an even function of the vortex core position around the equilibrium position. For small displacements $\vec{X}$ from the equilibrium, it can be thus given by a parabolic function of the vortex core position $X(x, y, 0)$ i.e. $U(\vec{X})=\frac{1}{2} k X^{2}$, where the restoring force constant $\mathrm{k}$ is related to the initial susceptibility.

The gyrocoupling coefficient $G$ and the dissipation coefficient D are constant irrespective of the vortex position. The force equation can be written with the vortex core position $X(x, y, 0)$ as

$$
G(\dot{x} \hat{j}-\dot{y} \hat{i})-k(x \hat{i}-y \hat{j})+D(\dot{x} \hat{i}-\dot{y} \hat{j})=0
$$

decoupling the $\hat{i}$ and $\hat{j}$ components results in

$$
\left\{\begin{array}{l}
-G \dot{y}+D x-k x=0, \\
-G \dot{x}+D y-k y=0
\end{array}\right.
$$

eliminating y from the above coupled equations, we get

$$
\ddot{x}+2 \eta \dot{x}+\omega^{2} x=0
$$

which is equivalent to the equation of a damped harmonic oscillator with dissipation coefficient and natural frequency given by

$$
\left\{\begin{array}{l}
\eta=-\frac{k D}{\left(G^{2}+D^{2}\right)}, \\
\omega_{0}^{2}=\frac{k^{2}}{\left(G^{2}+D^{2}\right)}
\end{array}\right.
$$

Thus, the experimental gyrotropic vortex core motion of the circular (or spiral) rotation can be explained. As a direct result, the core will be free of inertia. When covering vortex core switching, this assumption obviously becomes untrue, as a rigid vortex core is not compatible with the model of vortex core switching by anti-vortex vortex pair creation. 


\section{APPENDIX B \\ SMF Equation}

In a ferromagnet the electron spin interacts with the local magnetization (unit vector $\boldsymbol{m})$ via the exchange interaction and the corresponding interaction Hamiltonian is given by

$$
\mathcal{H}=\frac{\boldsymbol{p}^{2}}{2 m_{e}}-J_{e x} \boldsymbol{\sigma} \cdot \boldsymbol{m}(\mathbf{x}, t),
$$

where $\boldsymbol{p}, m_{e}, J_{e x}$ and $\boldsymbol{\sigma}$ are the momentum operator, the electron mass, the exchange coupling energy and the Pauli's matrices, respectively. The equation of motion of an electron can be obtained by the Heisenberg equation as

$$
\begin{aligned}
f_{i} & =m \ddot{x}_{i} \\
& =\frac{m}{(i \hbar)^{2}}\left[\left[x_{i}, \mathcal{H}\right], \mathcal{H}\right] \\
& =J_{e x} \nabla_{i}(\boldsymbol{\sigma} \cdot \boldsymbol{m})
\end{aligned}
$$

where $x_{i}$ and $f_{i}$ are the $i$-components $(i=x, y, z)$ of the position and "force" operators for the electron, respectively. The net force acting upon the electron is

$$
\left\langle f_{i}\right\rangle=J\langle\boldsymbol{\sigma}\rangle \cdot \nabla_{i} \boldsymbol{m}
$$

Here $\langle\boldsymbol{\sigma}\rangle$ is the unit vector of the electron spin direction. The magnetization $\boldsymbol{m}$ dynamics can be well described by the earlier discussed phenomenological LLG equation. The remaining work is to determine the electron spin $\langle\boldsymbol{\sigma}\rangle$ dynamics, which prefers to be parallel to the magnetization $\boldsymbol{m}$ due to the strong exchange interaction between them. In general, the electron spin $\langle\boldsymbol{\sigma}\rangle$ is expressed as $\langle\boldsymbol{\sigma}\rangle=a \boldsymbol{m}+b \delta \boldsymbol{m}$, where $\delta \boldsymbol{m}$ is a unit vector perpendicular to $\boldsymbol{m}$, and $a$ and $b$ are coefficients $(|a| \gg$ $|b|)$. The component am does not contribute to the force (B.3), because $\boldsymbol{m} \cdot \nabla_{i} \boldsymbol{m}=$ 0 . In other words, if the electron spin is exactly parallel to the magnetization at every time and space, the electron's energy is the same always and everywhere, and the electron does not feel any force. This means that what causes the force is the off-diagonal component of the electron spin with respect to the magnetization, i.e., the misalignment between them. 
According to the Heisenberg equation, the equation of motion of the electron spin is

$$
\partial_{t}\langle\boldsymbol{\sigma}\rangle=\frac{J_{e x} \hbar}{2}\langle\boldsymbol{\sigma}\rangle \times \boldsymbol{m}(\mathbf{x}, t) .
$$

This expression shows the Larmor precession of the conduction electron spin around the magnetization. Actually, in addition to the precession torque, the electron spin feels a damping torque to the magnetization. To obtain an analytical form of the electron spin dynamics without solving Eq. (B.4) in a straightforward manner, let us consider the problem in a more convenient coordinate system in which the magnetization is parallel to the $z$-axis at every time and space. Such an operation is realized by a $\mathrm{SU}(2)$ local gauge transformation $\psi \mapsto U \psi$, where $U=$ $\exp \left(i \sigma_{y} \theta(x, t) / 2\right) \exp \left(i \sigma_{z} \varphi(x, t) / 2\right), \theta$ and $\varphi$ are defined by $\boldsymbol{m}=(\sin \theta \cos \varphi, \sin \theta \sin \varphi, \cos \theta)$, and $\psi$ is a two component spinor.

After performing the gauge transformation, the Hamiltonian in the rotational frame is

$$
\begin{aligned}
\mathcal{H}^{\prime} & =U \mathcal{H} U^{\dagger}-i \hbar U\left(\partial_{t} U^{\dagger}\right) \\
& =\frac{\left(\boldsymbol{p}-i \hbar U\left(\nabla U^{\dagger}\right)\right)^{2}}{2 m}-J \sigma_{z}-i \hbar U\left(\partial_{t} U^{\dagger}\right) \\
& =\frac{\left(\boldsymbol{p}-\sigma_{x} \boldsymbol{A}_{x}-\sigma_{y} \boldsymbol{A}_{y}-\sigma_{z} \boldsymbol{A}_{z}\right)^{2}}{2 m}-J \sigma_{z}-i \hbar U\left(\partial_{t} U^{\dagger}\right) \\
& =\frac{\boldsymbol{p}^{2}+\boldsymbol{A}_{x}^{2}+\boldsymbol{A}_{y}^{2}+\boldsymbol{A}_{z}^{2}}{2 m}-\boldsymbol{\sigma} \cdot\left(J \boldsymbol{m}^{\prime}+\boldsymbol{d}^{\prime}\right),
\end{aligned}
$$

where

$$
\begin{aligned}
\boldsymbol{d}^{\prime}= & \frac{\hbar}{2}\left(\boldsymbol{m} \times \partial_{t} \boldsymbol{m}\right)^{\prime} \\
& +\frac{\hbar \cos \theta \partial_{t} \varphi}{2} \boldsymbol{m}^{\prime}+\frac{1}{2 m}\left[\begin{array}{l}
\boldsymbol{p} \cdot \boldsymbol{A}_{x}+\boldsymbol{A}_{x} \cdot \boldsymbol{p} \\
\boldsymbol{p} \cdot \boldsymbol{A}_{y}+\boldsymbol{A}_{y} \cdot \boldsymbol{p} \\
\boldsymbol{p} \cdot \boldsymbol{A}_{z}+\boldsymbol{A}_{z} \cdot \boldsymbol{p}
\end{array}\right] .
\end{aligned}
$$

The SU(2) pure gauge fields, $i \hbar U\left(\nabla U^{\dagger}\right)=\sigma_{x} \boldsymbol{A}_{x}+\sigma_{y} \boldsymbol{A}_{y}+\sigma_{z} \boldsymbol{A}_{z}$ and $-i \hbar U\left(\partial_{t} U^{\dagger}\right)$, arise as a result of the gauge transformation. The superscript ' denotes a vector in the rotational frame, for instance, $\boldsymbol{m}^{\prime}=(0,0,1)$.

Equation (B.5) indicates that when the magnetization depends upon time, the electron spin feels the effective magnetic field $J \boldsymbol{m}^{\prime}+\boldsymbol{d}^{\prime}$ in the rotating frame. Therefore, in reality, the electron spin precesses around the effective magnetic field $\boldsymbol{J} \boldsymbol{m}^{\prime}+\boldsymbol{d}^{\prime}$ rather than the magnetization $\boldsymbol{J}^{\prime}$. If the magnetic texture is smooth 
and slow enough, we can adopt an adiabatic approximation in which $\langle\boldsymbol{\sigma}\rangle$ is approximated to be parallel or antiparallel to the effective magnetic field at every time and space; a normalized electron spin is

$$
\begin{aligned}
\langle\boldsymbol{\sigma}\rangle_{\uparrow \downarrow}^{\prime}= & \pm \boldsymbol{m}^{\prime} \pm \frac{\hbar}{2 J}\left(\boldsymbol{m} \times \partial_{t} \boldsymbol{m}\right)^{\prime} \\
& \pm \frac{\hbar \cos \theta \partial_{t} \varphi}{2 J} \boldsymbol{m}^{\prime} \pm \frac{1}{m J}\left[\begin{array}{c}
\boldsymbol{A}_{x} \cdot m \boldsymbol{v} \\
\boldsymbol{A}_{y} \cdot m \boldsymbol{v} \\
\boldsymbol{A}_{z} \cdot m \boldsymbol{v}
\end{array}\right]
\end{aligned}
$$

where $\uparrow(\downarrow)$ stands for the majority (minority) spin with respect to the local magnetization and corresponds to $+(-)$ in the right-hand side. Here, we replace $\boldsymbol{p} \cdot \boldsymbol{A}_{i}+\boldsymbol{A}_{i} \cdot \boldsymbol{p}$ by $2 \boldsymbol{A}_{i} \cdot m \boldsymbol{v}$, where $\boldsymbol{v}$ is the averaged velocity vector of electrons. The expression for $\langle\boldsymbol{\sigma}\rangle$ in the laboratory frame is obtained by the $O(3)$ rotation as

$$
\begin{aligned}
\langle\boldsymbol{\sigma}\rangle_{\uparrow \downarrow} & =\left[\begin{array}{ccc}
\cos \theta \cos \varphi & -\sin \varphi & \sin \theta \cos \theta \\
\cos \theta \sin \varphi & \cos \varphi & \sin \theta \sin \varphi \\
-\sin \theta & 0 & \cos \theta
\end{array}\right]\langle\boldsymbol{\sigma}\rangle_{\uparrow \downarrow}^{\prime} \\
& = \pm(\boldsymbol{m}+\boldsymbol{d} / J) .
\end{aligned}
$$

That is, the electron spin has the off-diagonal component with respect to the magnetization, $\boldsymbol{d} / J$ (FIG. ??).

By substituting Eq. (B.8) to Eq. (B.3), the force can be expressed only by the magnetization dynamics as

$$
\left\langle f_{i}\right\rangle_{\uparrow \downarrow}= \pm \frac{\hbar}{2}\left(\boldsymbol{m} \times \partial_{t} \boldsymbol{m}\right) \cdot \nabla_{i} \boldsymbol{m}-e \boldsymbol{v} \times \boldsymbol{B}_{\uparrow \downarrow},
$$

where

$$
\left(\boldsymbol{B}_{\uparrow \downarrow}\right)_{i}=\mp \frac{\hbar}{4 e} \varepsilon^{i j k} \boldsymbol{m} \cdot\left(\nabla_{j} \boldsymbol{m} \times \nabla_{k} \boldsymbol{m}\right) .
$$

The first term in Eq. (B.9) is a spin dependent driving force, which contributes to the spin-motive force. On the other hand, the second term in Eq. (B.9) is the Lorentz type force due to the spin dependent magnetic field $\boldsymbol{B}_{\uparrow \downarrow}$, produced by the non-coplanar magnetization configuration as Eq. (B.10), and the transverse conductivity appears, i.e., the anomalous Hall effect. From Eq. (B.9), one expects a large SMF signal when fast dynamics is induced on the magnetic structure with strong modulation. It has already been shown theoretically and experimentally that the diameter of the vortex core is of the order of $10 \mathrm{~nm}$ and the velocity of the core dynamics is in the range of $100 \mathrm{~m} / \mathrm{s}$. Furthermore, switching occurs on a time scale of picoseconds. These conditions give us a possibility of observing a large spin motive force signal in vortex structures. 


\section{Bibliography}

[1] June W. Lau and Justin M. Shaw. Magnetic nanostructures for advanced technologies: fabrication, metrology and challenges. Journal of Physics D: Applied Physics, 44:303001, 2011.

[2] S. D. Bader. Colloquium : Opportunities in nanomagnetism. Rev. Mod. Phys., 78:1, 2006.

[3] Alberto P. Guimarães. Principles of Nanomagnetism. Springer Berlin Heidelberg, 2009.

[4] C. A. Ross. Patterned magnetic recording media. Annual Review of Materials Research, 31:203, 2001.

[5] Riccardo Hertel, Wulf Wulfhekel, and Jürgen Kirschner. Domain-wall induced phase shifts in spin waves. Phys. Rev. Lett., 93:257202, 2004.

[6] Claude Chappert, Albert Fert, and Frédéric Nguyen Van Dau. The emergence of spin electronics in data storage. Nat. Mat., 6:813, 2007.

[7] Stuart S. P. Parkin, Masamitsu Hayashi, and Luc Thomas. Magnetic domainwall racetrack memory. Science, 320:190, 2008.

[8] M. M. Miller, G. A. Prinz, S.-F. Cheng, and S. Bounnak. Detection of a micron-sized magnetic sphere using a ring-shaped anisotropic magnetoresistance-based sensor: A model for a magnetoresistance-based biosensor. Appl. Phys. Lett., 81:2211, 2002.

[9] Skumryev Vassil, Stoyanov Stoyan, Zhang Yong, Hadjipanayis George, Givord Dominique, and Nogues Josep. Beating the superparamagnetic limit with exchange bias. Nature, 423:850, 2003.

[10] R. P. Cowburn, D. K. Koltsov, A. O. Adeyeye, M. E. Welland, and D. M. Tricker. Single-domain circular nanomagnets. Phys. Rev. Lett., 83:1042, 1999.

[11] P. Vavassori, V. Metlushko, B. Ilic, M. Gobbi, M. Donolato, M. Cantoni, and R. Bertacco. Domain wall displacement in Py square ring for single nanometric magnetic bead detection. Appl. Phys. Lett., 93:203502, 2008. 
[12] V. S. Pribiag, I. N. Krivorotov, G. D. Fuchs, P. M. Braganca, O. Ozatay, J. C. Sankey, D. C. Ralph, and R. A. Buhrman. Magnetic vortex oscillator driven by d.c. spin-polarized current. Nat. Phys., 3:498, 2007.

[13] Konstantin Y. Guslienko. Spin torque induced magnetic vortex dynamics in layered nanopillars. Journal of Spintronics and Magnetic Nanomaterials, $1: 70,2012$.

[14] V. E. Demidov, H. Ulrichs, S. Urazhdin, S. O. Demokritov, V. Bessonov, R. Gieniusz, and A. Maziewski. Resonant frequency multiplication in microscopic magnetic dots. Appl. Phys. Lett., 99:012505, 2011.

[15] J. Raabe, R. Pulwey, R. Sattler, T. Schweinböck, J. Zweck, and D. Weiss. Magnetization pattern of ferromagnetic nanodisks. J. .Appl. Phys., 88:4437, 2000 .

[16] T. Shinjo, T. Okuno, R. Hassdorf, K. Shigeto, and T. Ono. Magnetic vortex core observation in circular dots of permalloy. Science, 289:930, 2000.

[17] L. D. Landau and E. M. Lifshitz. Theory of the dispersion of magnetic permeability in ferromagnetic bodies. Phys. Z. Sowjetunion, 8:153, 1935.

[18] T. L. Gilbert. A phenomenological theory of damping in ferromagnetic materials. Magnetics, IEEE Transactions on, 40:3443, 2004.

[19] A. A. Thiele. Steady-state motion of magnetic domains. Phys. Rev. Lett., 30:230, 1973.

[20] D. C. Ralph and M. D. Stiles. Spin transfer torques. Journal of Magnetism and Magnetic Materials, 320:1190, 2008.

[21] L. Berger. Exchange interaction between ferromagnetic domain wall and electric current in very thin metallic films. J. Appl. Phys., 55:1954, 1984.

[22] J. C. Slonczewski. Current-driven excitation of magnetic multilayers. Journal of Magnetism and Magnetic Materials, 159:L1, 1996.

[23] Z. Li and S. Zhang. Domain-wall dynamics and spin-wave excitations with spin-transfer torques. Phys. Rev. Lett., 92:207203, 2004.

[24] A. Thiaville, Y. Nakatani, J. Miltat, and N. Vernier. Domain wall motion by spin-polarized current: a micromagnetic study. J. Appl. Phys., 95:7049, 2004. 
[25] A. Thiaville, Y. Nakatani, J. Miltat, and Y. Suzuki. Micromagnetic understanding of current-driven domain wall motion in patterned nanowires. EPL (Europhysics Letters), 69:990, 2005.

[26] Jiang Xiao, A. Zangwill, and M. D. Stiles. Spin-transfer torque for continuously variable magnetization. Phys. Rev. B, 73:054428, 2006.

[27] Yaroslav Tserkovnyak, Arne Brataas, and Gerrit E. W. Bauer. Theory of current-driven magnetization dynamics in inhomogeneous ferromagnets. Journal of Magnetism and Magnetic Materials, 320:1282, 2008.

[28] S. Zhang and Z. Li. Roles of nonequilibrium conduction electrons on the magnetization dynamics of ferromagnets. Phys. Rev. Lett., 93:127204, 2004.

[29] Arne Brataas, Andrew D. Kent, and Hideo Ohno. Current-induced torques in magnetic materials. Nat. Mat., 11:372, 2012.

[30] G. S. D. Beach, C. Knutson, C. Nistor, M. Tsoi, and J. L. Erskine. Nonlinear domain-wall velocity enhancement by spin-polarized electric current. Phys. Rev. Lett., 97:057203, 2006.

[31] Masamitsu Hayashi, Luc Thomas, Charles Rettner, Rai Moriya, and Stuart S. P. Parkin. Direct observation of the coherent precession of magnetic domain walls propagating along permalloy nanowires. Nat. Phys., 3:21, 2007.

[32] G. S. D. Beach, M. Tsoi, and J. L. Erskine. Current-induced domain wall motion. Journal of Magnetism and Magnetic Materials, 320:1272, 2008.

[33] J.-Y. Chauleau, H. G. Bauer, H. S. Körner, J. Stigloher, M. Härtinger, G. Woltersdorf, and C. H. Back. Self-consistent determination of the key spin-transfer torque parameters from spin-wave doppler experiments. Phys. Rev. B, 89:020403, 2014.

[34] M. E. Gouva, G. M. Wysin, A. R. Bishop, and F. G. Mertens. Vortices in the classical two-dimensional anisotropic heisenberg model. Phys. Rev. B, 39:11840, 1989.

[35] Jonathan Kin Ha, Riccardo Hertel, and J. Kirschner. Micromagnetic study of magnetic configurations in submicron permalloy disks. Phys. Rev. B, 67:224432, 2003. 
[36] A. Wachowiak, J. Wiebe, M. Bode, O. Pietzsch, M. Morgenstern, and R. Wiesendanger. Direct observation of internal spin structure of magnetic vortex cores. Science (New York, N.Y.), 298:577, 2002.

[37] J. Miltat and A. Thiaville. Vortex cores-smaller than small. Science, 298:555, 2002 .

[38] R. P. Cowburn. Magnetic nanodots for device applications. Journal of Magnetism and Magnetic Materials, 242:505, 2002.

[39] Stellan Bohlens, Benjamin Krüger, André Drews, Markus Bolte, Guido Meier, and Daniela Pfannkuche. Current controlled random-access memory based on magnetic vortex handedness. Appl. Phys. Lett., 93:142508, 2008.

[40] Kunihiro Nakano, Kenji Tanabe, Ryo Hiramatsu, Daichi Chiba, Norikazu Ohshima, Shinya Kasai, Tomonori Sato, Yoshinobu Nakatani, Koji Sekiguchi, Kensuke Kobayashi, and Teruo Ono. Real-time observation of electrical vortex core switching. Appl. Phys. Lett., 102:072405, 2013.

[41] Hauke H. Langner, Lars Bocklage, Toru Matsuyama, and Guido Meier. Inductive detection of magnetic vortex gyration. Phys. Rev. B, 87:064420, 2013.

[42] K. Yu. Guslienko, B. A. Ivanov, V. Novosad, Y. Otani, H. Shima, and K. Fukamichi. Eigenfrequencies of vortex state excitations in magnetic submicron-size disks. J. Appl. Phys., 91:8037, 2002.

[43] Denis D. Sheka, Juan P. Zagorodny, Jean-Guy Caputo, Yuri Gaididei, and Franz G. Mertens. Vortex motion in a finite-size easy-plane ferromagnet and application to a nanodot. Phys. Rev. B, 71:134420, 2005.

[44] K. Yu. Guslienko, W. Scholz, R. W. Chantrell, and V. Novosad. Vortex-state oscillations in soft magnetic cylindrical dots. Phys. Rev. B, 71:144407, 2005.

[45] K. Yu. Guslienko, X. F. Han, D. J. Keavney, R. Divan, and S. D. Bader. Magnetic vortex core dynamics in cylindrical ferromagnetic dots. Phys. Rev. Lett., 96:067205, 2006.

[46] Ki-Suk Lee and Sang-Koog Kim. Gyrotropic linear and nonlinear motions of a magnetic vortex in soft magnetic nanodots. Appl. Phys. Lett., 91:132511, 2007. 
[47] Benjamin Krüger, André Drews, Markus Bolte, Ulrich Merkt, Daniela Pfannkuche, and Guido Meier. Harmonic oscillator model for current- and field-driven magnetic vortices. Phys. Rev. B, 76:224426, 2007.

[48] D. L. Huber. Dynamics of spin vortices in two-dimensional planar magnets. Phys. Rev. B, 26:3758, 1982.

[49] A. Vansteenkiste, K. W. Chou, M. Weigand, M. Curcic, V. Sackmann, H. Stoll, T. Tyliszczak, G. Woltersdorf, C. H. Back, G. Schütz, and B. Van Waeyenberge. X-ray imaging of the dynamic magnetic vortex core deformation. Nat. Phys., 5:332, 2009.

[50] Junya Shibata, Yoshinobu Nakatani, Gen Tatara, Hiroshi Kohno, and Yoshichika Otani. Current-induced magnetic vortex motion by spin-transfer torque. Phys. Rev. B, 73:020403, 2006.

[51] Markus Bolte, Guido Meier, Benjamin Krüger, André Drews, René Eiselt, Lars Bocklage, Stellan Bohlens, Tolek Tyliszczak, Arne Vansteenkiste, Bartel Van Waeyenberge, Kang Wei Chou, Aleksandar Puzic, and Hermann Stoll. Time-resolved x-ray microscopy of spin-torque-induced magnetic vortex gyration. Phys. Rev. Lett., 100:176601, 2008.

[52] Benjamin Krüger, Massoud Najafi, Stellan Bohlens, Robert Frömter, Dietmar P. F. Möller, and Daniela Pfannkuche. Proposal of a robust measurement scheme for the nonadiabatic spin torque using the displacement of magnetic vortices. Phys. Rev. Lett., 104:077201, 2010.

[53] Yoshinobu Nakatani and Teruo Ono. Effect of the oersted field on a vortex core switching by pulse spin current. Appl. Phys. Lett., 99:122509, 2011.

[54] N. Kikuchi, S. Okamoto, O. Kitakami, Y. Shimada, S. G. Kim, Y. Otani, and K. Fukamichi. Vertical bistable switching of spin vortex in a circular magnetic dot. J. Appl. Phys., 90, 2001.

[55] André Thiaville, José Miguel García, Rok Dittrich, Jacques Miltat, and Thomas Schrefl. Micromagnetic study of bloch-point-mediated vortex core reversal. Phys. Rev. B, 67:094410, 2003.

[56] V.P. Kravchuk and D.D. Sheka. Thin ferromagnetic nanodisk in transverse magnetic field. Physics of the Solid State, 49:1923, 2007. 
[57] R. Hertel, S. Gliga, M. Fähnle, and C. M. Schneider. Ultrafast nanomagnetic toggle switching of vortex cores. Phys. Rev. Lett., 98:117201, 2007.

[58] Keisuke Yamada, Shinya Kasai, Yoshinobu Nakatani, Kensuke Kobayashi, Hiroshi Kohno, Andre Thiaville, and Teruo Ono. Electrical switching of the vortex core in a magnetic disk. Nat. Mat., 6:270, 2007.

[59] Y. B. Gaididei, V. P. Kravchuk, D. D. Sheka, and F. G. Mertens. Switching phenomena in magnetic vortex dynamics. Low Temperature Physics, 34:528, 2008.

[60] Yuri Gaididei, Till Kamppeter, Franz G. Mertens, and A. R. Bishop. Switching between different vortex states in two-dimensional easy-plane magnets due to an ac magnetic field. Phys. Rev. B, 61:9449, 2000.

[61] A. S. Kovalev and J. E. Prilepsky. Mechanism of vortex switching in magnetic nanodots under a circular magnetic field. i. resonance action of the field on the nanodot eigenmodes. Low Temperature Physics, 28:921, 2002.

[62] Zagorodny, J. P., Gaididei, Y., Mertens, F. G., and Bishop, A. R. Switching of vortex polarization in 2d easy-plane magnets by magnetic fields. Eur. Phys. J. B, 31:471, 2003.

[63] B. Van Waeyenberge, A. Puzic, H. Stoll, K. W. Chou, T. Tyliszczak, R. Hertel, M. Fähnle, H. Bruckl, K. Rott, G. Reiss, I. Neudecker, D. Weiss, C. H. Back, and G. Schütz. Magnetic vortex core reversal by excitation with short bursts of an alternating field. Nature, 444:461, 2006.

[64] Matthias Noske, Hermann Stoll, Manfred Fähnle, Riccardo Hertel, and Gisela Schütz. Mechanisms for the symmetric and antisymmetric switching of a magnetic vortex core: Differences and common aspects. Phys. Rev. B, 91:014414, 2015 .

[65] Ki-Suk Lee, SangKook Choi, and Sang-Koog Kim. Radiation of spin waves from magnetic vortex cores by their dynamic motion and annihilation processes. Appl. Phys. Lett., 87:192502, 2005.

[66] Sangkook Choi, Ki-Suk Lee, Konstantin Yu. Guslienko, and Sang-Koog Kim. Strong radiation of spin waves by core reversal of a magnetic vortex and their wave behaviors in magnetic nanowire waveguides. Phys. Rev. Lett., 98:087205, 2007. 
[67] Keisuke Yamada, Shinya Kasai, Yoshinobu Nakatani, Kensuke Kobayashi, and Teruo Ono. Switching magnetic vortex core by a single nanosecond current pulse. Appl. Phys. Lett., 93:152502, 2008.

[68] Markus Weigand, Bartel Van Waeyenberge, Arne Vansteenkiste, Michael Curcic, Vitalij Sackmann, Hermann Stoll, Tolek Tyliszczak, Konstantine Kaznatcheev, Drew Bertwistle, Georg Woltersdorf, Christian H. Back, and Gisela Schütz. Vortex core switching by coherent excitation with single in-plane magnetic field pulses. Phys. Rev. Lett., 102:077201, 2009.

[69] Volodymyr P. Kravchuk, Denis D. Sheka, Yuri Gaididei, and Franz G. Mertens. Controlled vortex core switching in a magnetic nanodisk by a rotating field. J. Appl. Phys., 102:043908, 2007.

[70] Sang-Koog Kim, Ki-Suk Lee, Young-Sang Yu, and Youn-Seok Choi. Reliable low-power control of ultrafast vortex-core switching with the selectivity in an array of vortex states by in-plane circular-rotational magnetic fields and spin-polarized currents. Appl. Phys. Lett., 92:022509, 2008.

[71] Michael Curcic, Hermann Stoll, Markus Weigand, Vitalij Sackmann, Patrick Juellig, Matthias Kammerer, Matthias Noske, Markus Sproll, Bartel Van Waeyenberge, Arne Vansteenkiste, Georg Woltersdorf, Tolek Tyliszczak, and Gisela Schütz. Magnetic vortex core reversal by rotating magnetic fields generated on micrometer length scales. Physica Status Solidi (b), 248:2317, 2011.

[72] Young-Sang Yu, Ki-Suk Lee, Hyunsung Jung, Youn-Seok Choi, MyoungWoo Yoo, Dong-Soo Han, Mi-Young Im, Peter Fischer, and Sang-Koog Kim. Polarization-selective vortex-core switching by tailored orthogonal gaussianpulse currents. Phys. Rev. B, 83:174429, 2011.

[73] Conyers Herring and Charles Kittel. On the theory of spin waves in ferromagnetic media. Phys. Rev., 81:869, 1951.

[74] Sebastian Neusser and Dirk Grundler. Magnonics: Spin waves on the nanoscale. Advanced Materials, 21:2927, 2009.

[75] Dr. Kamel Ounadjela Prof. Burkard Hillebrands. Spin Dynamics in Confined Magnetic Structures I. Springer Berlin Heidelberg, 2002. 
[76] B. A. Kalinikos and A. N. Slavin. Theory of dipole-exchange spin wave spectrum for ferromagnetic films with mixed exchange boundary conditions. Journal of Physics C: Solid State Physics, 19:7013, 1986.

[77] S. O. Demokritov, B. Hillebrands, and A. N. Slavin. Brillouin light scattering studies of confined spin waves: linear and nonlinear confinement. Physics Reports, 348:441, 2001.

[78] M. Sproll. Schalten der Polarität magnetischer Vortexkerne durch eine ZweiFrequenzen Anregung und mittels direkter Einkopplung eines Stroms. PhD thesis, Max - Planck - Institut für Intelligente Systeme, Stuttgart, 2014.

[79] Hans G. Bauer, Markus Sproll, Christian H. Back, and Georg Woltersdorf. Vortex core reversal due to spin wave interference. Phys. Rev. Lett., 112:077201, 2014.

[80] Markus Sproll, Matthias Noske, Hans Bauer, Matthias Kammerer, Ajay Gangwar, Georg Dieterle, Markus Weigand, Hermann Stoll, Georg Woltersdorf, Christian H. Back, and Gisela Schütz. Low-amplitude magnetic vortex core reversal by non-linear interaction between azimuthal spin waves and the vortex gyromode. Appl. Phys. Lett., 104:012409, 2014.

[81] R.W. Damon and J.R. Eshbach. Magnetostatic modes of a ferromagnet slab. Journal of Physics and Chemistry of Solids, 19:308, 1961.

[82] Handbook of Magnetism and Advanced Magnetic Materials, Volume 1 and 2.

[83] C. E. Zaspel, B. A. Ivanov, J. P. Park, and P. A. Crowell. Excitations in vortex-state permalloy dots. Phys. Rev. B, 72:024427, 2005.

[84] M. Helsen, A. Gangwar, J. De Clercq, A. Vansteenkiste, M. Weigand, C. H. Back, and B. Van Waeyenberge. Non-linear radial spinwave modes in thin magnetic disks. Appl. Phys. Lett., 106:032405, 2015.

[85] K. Yu. Guslienko and A. N. Slavin. Spin-waves in cylindrical magnetic dot arrays with in-plane magnetization. J. Appl. Phys., 87:6337, 2000.

[86] C. Bayer, J. Jorzick, B. Hillebrands, S. O. Demokritov, R. Kouba, R. Bozinoski, A. N. Slavin, K. Y. Guslienko, D. V. Berkov, N. L. Gorn, and M. P. Kostylev. Spin-wave excitations in finite rectangular elements of $N i_{80} F e_{20}$. Phys. Rev. B, 72:064427, 2005. 
[87] M. Buess, R. Höllinger, T. Haug, K. Perzlmaier, U. Krey, D. Pescia, M. R. Scheinfein, D. Weiss, and C. H. Back. Fourier transform imaging of spin vortex eigenmodes. Phys. Rev. Lett., 93:077207, 2004.

[88] M. Buess, T. P. J. Knowles, R. Höllinger, T. Haug, U. Krey, D. Weiss, D. Pescia, M. R. Scheinfein, and C. H. Back. Excitations with negative dispersion in a spin vortex. Phys. Rev. B, 71:104415, 2005.

[89] Matthias Kammerer, Markus Weigand, Michael Curcic, Matthias Noske, Markus Sproll, Arne Vansteenkiste, Bartel Van Waeyenberge, Hermann Stoll, Georg Woltersdorf, Christian H Back, and Gisela Schuetz. Magnetic vortex core reversal by excitation of spin waves. Nature Communications, 2:279, 2011.

[90] Matthias Kammerer, Hermann Stoll, Matthias Noske, Markus Sproll, Markus Weigand, Christian Illg, Georg Woltersdorf, Manfred Fähnle, Christian Back, and Gisela Schütz. Fast spin-wave-mediated magnetic vortex core reversal. Phys. Rev. B, 86:134426, 2012.

[91] Matthias Noske, Ajay Gangwar, Hermann Stoll, Matthias Kammerer, Markus Sproll, Georg Dieterle, Markus Weigand, Manfred Fähnle, Georg Woltersdorf, Christian H. Back, and Gisela Schütz. Unidirectional sub-100-ps magnetic vortex core reversal. Phys. Rev. B, 90:104415, 2014.

[92] V. Novosad, F. Y. Fradin, P. E. Roy, K. S. Buchanan, K. Yu. Guslienko, and S. D. Bader. Magnetic vortex resonance in patterned ferromagnetic dots. Phys. Rev. B, 72:024455, 2005.

[93] V. Novosad, M. Grimsditch, K. Yu. Guslienko, P. Vavassori, Y. Otani, and S. D. Bader. Spin excitations of magnetic vortices in ferromagnetic nanodots. Phys. Rev. B, 66:052407, 2002.

[94] K. Vogt, O. Sukhostavets, H. Schultheiss, B. Obry, P. Pirro, A. A. Serga, T. Sebastian, J. Gonzalez, K. Y. Guslienko, and B. Hillebrands. Optical detection of vortex spin-wave eigenmodes in microstructured ferromagnetic disks. Phys. Rev. B, 84:174401, 2011.

[95] G. Schütz, W. Wagner, W. Wilhelm, P. Kienle, R. Zeller, R. Frahm, and G. Materlik. Absorption of circularly polarized x rays in iron. Phys. Rev. Lett., 58:737, 1987. 
[96] Shinya Kasai, Yoshinobu Nakatani, Kensuke Kobayashi, Hiroshi Kohno, and Teruo Ono. Current-driven resonant excitation of magnetic vortices. Phys. Rev. Lett., 97:107204, 2006.

[97] Rai Moriya, Luc Thomas, Masamitsu Hayashi, Yaroslaw B. Bazaliy, Charles Rettner, and Stuart S. P. Parkin. Probing vortex-core dynamics using currentinduced resonant excitation of a trapped domain wall. Nat. Phys., 4:368, 2008.

[98] Yaroslav Tserkovnyak and Matthew Mecklenburg. Electron transport driven by nonequilibrium magnetic textures. Phys. Rev. B, 77:134407, 2008.

[99] Dahai Wei, Martin Obstbaum, Mirko Ribow, Christian H Back, and Georg Woltersdorf. Spin Hall voltages from a.c. and d.c. spin currents. Nat Commun, 5:3768, 2014.

[100] M. Obstbaum, M. Härtinger, H. G. Bauer, T. Meier, F. Swientek, C. H. Back, and G. Woltersdorf. Inverse spin hall effect in $N i_{81} F e_{19} /$ normal-metal bilayers. Phys. Rev. B, 89:060407, 2014.

[101] W. Thomson. On the electro-dynamic qualities of metals : Effects of magnetization on the electric conductivity of nickel and of iron. Proceedings of the Royal Society of London, 8:546, 1856.

[102] R. M. Bozorth. Magnetoresistance and domain theory of iron-nickel alloys. Phys. Rev., 70:923, 1946.

[103] J. Smit. Magnetoresistance of ferromagnetic metals and alloys at low temperatures. Physica, 17:612, 1951.

[104] L. Berger. Influence of spin-orbit interaction on the transport processes in ferromagnetic nickel alloys, in the presence of a degeneracy of the $3 \mathrm{~d}$ band. Physica, 30:1141, 1964.

[105] T. R. McGuire and R. I. Potter. Anisotropic magnetoresistance in ferromagnetic 3d alloys. Magnetics, IEEE Transactions on, 11:1018, 1975.

[106] Nils Kuhlmann, Andreas Vogel, and Guido Meier. Magnetization dynamics and cone angle precession in permalloy rectangles. Phys. Rev. B, 85:014410, 2012 . 
[107] Satoshi Sugimoto, Norinobu Hasegawa, Yasuhiro Niimi, Yasuhiro Fukuma, Shinya Kasai, and YoshiChika Otani. Detection of a symmetric circular gyration of the vortex core via the second-order harmonic magnetoresistance oscillation. Applied Physics Express, 7:023006, 2014.

[108] S. E. Barnes and S. Maekawa. Generalization of faraday's law to include nonconservative spin forces. Phys. Rev. Lett., 98:246601, 2007.

[109] Yuta Yamane, Jun'ichi Ieda, Jun-ichiro Ohe, Stewart E. Barnes, and Sadamichi Maekawa. Equation-of-motion approach of spin-motive force. $J$. Appl. Phys., 109:07C735, 2011.

[110] Jun-ichiro Ohe, Stewart E. Barnes, Hyun-Woo Lee, and Sadamichi Maekawa. Electrical measurements of the polarization in a moving magnetic vortex. Appl. Phys. Lett., 95:123110, 2009.

[111] M. E. Lucassen, G. C. F. L. Kruis, R. Lavrijsen, H. J. M. Swagten, B. Koopmans, and R. A. Duine. Spin motive forces due to magnetic vortices and domain walls. Phys. Rev. B, 84:014414, 2011.

[112] K. Tanabe, D. Chiba, J. Ohe, S. Kasai, H. Kohno, S. E. Barnes, S. Maekawa, K. Kobayashi, and T. Ono. Spin-motive force due to a gyrating magnetic vortex. 3:845, 2012.

[113] J. Nogués, J. Sort, V. Langlais, V. Skumryev, S. Suriñach, J.S. Muñoz, and M.D. Baró. Exchange bias in nanostructures. Physics Reports, 422:65, 2005.

[114] J. Sort, K. S. Buchanan, V. Novosad, A. Hoffmann, G. Salazar-Alvarez, A. Bollero, M. D. Baró, B. Dieny, and J. Nogués. Imprinting vortices into antiferromagnets. Phys. Rev. Lett., 97:067201, 2006.

[115] O. G. Heinonen, D. K. Schreiber, and A. K. Petford-Long. Micromagnetic modeling of spin-wave dynamics in exchange-biased permalloy disks. Phys. Rev. B, 76:144407, 2007.

[116] V. Cambel and G. Karapetrov. Control of vortex chirality and polarity in magnetic nanodots with broken rotational symmetry. Phys. Rev. B, 84:014424, 2011.

[117] V. Uhlir, M. Urbanek, L. Hladik, J. Spousta, M-Y Im, P. Fischer, N. Eibagi, J. J. Kan, E. E. Fullerton, and T. Sikola. Dynamic switching of the spin circulation in tapered magnetic nanodisks. Nat. Nano., 8:341, 2013. 
[118] J. I. Martin, J. Nogués, Kai Liu, J. L. Vicent, and Ivan K. Schuller. Ordered magnetic nanostructures: fabrication and properties. Journal of Magnetism and Magnetic Materials, 256:449, 2003.

[119] C. Vieu, F. Carcenac, A. Pépin, Y. Chen, M. Mejias, A. Lebib, L. ManinFerlazzo, L. Couraud, and H. Launois. Electron beam lithography: resolution limits and applications. Applied Surface Science, 164:111, 2000.

[120] Vitor R. Manfrinato, Lihua Zhang, Dong Su, Huigao Duan, Richard G. Hobbs, Eric A. Stach, and Karl K. Berggren. Resolution limits of electron-beam lithography toward the atomic scale. Nano Letters, 13:1555-1558, 2013.

[121] S. Neusser, G. Duerr, H. G. Bauer, S. Tacchi, M. Madami, G. Woltersdorf, G. Gubbiotti, C. H. Back, and D. Grundler. Anisotropic propagation and damping of spin waves in a nanopatterned antidot lattice. Phys. Rev. Lett., 105:067208, 2010.

[122] C. T. Chen, F. Sette, Y. Ma, and S. Modesti. Soft-x-ray magnetic circular dichroism at the $l_{2,3}$ edges of nickel. Phys. Rev. B, 42:7262, 1990.

[123] B. T. Thole, P. Carra, F. Sette, and G. van der Laan. X-ray circular dichroism as a probe of orbital magnetization. Phys. Rev. Lett., 68:1943, 1992.

[124] Akinobu Yamaguchi, Keiichi Motoi, Hideki Miyajima, Tomonori Sato, and Yoshinobu Nakatani. Nonlinear vortex motion induced by the simultaneous application of RF and DC currents in a micron-sized $F e_{19} N i_{81}$ disk. IEEE Transactions on Magnetics, 46:1994, 2010.

[125] R. L. Compton and P. A. Crowell. Dynamics of a pinned magnetic vortex. Phys. Rev. Lett., 97:137202, 2006.

[126] R. L. Compton, T. Y. Chen, and P. A. Crowell. Magnetic vortex dynamics in the presence of pinning. Phys. Rev. B, 81:144412, 2010.

[127] Hongki Min, R. D. McMichael, Jacques Miltat, and M. D. Stiles. Effects of disorder on magnetic vortex gyration. Phys. Rev. B, 83:064411, 2011.

[128] Satoshi Yakata, Terumitsu Tanaka, Kohei Kiseki, Kimihide Matsuyama, and Takashi Kimura. Wide range tuning of resonant frequency for a vortex core in a regular triangle magnet. Scientific reports, 3:3567, 2013. 
[129] M. Helsen, A. Gangwar, A. Vansteenkiste, and B. Van Waeyenberge. Magneto-optical spectrum analyzer. Rev. Sci. Inst., 85:083902, 2014. 